\title{
Prognostic and Prediction Modelling with Radiomics for Non-Small Cell Lung Cancer
}

Citation for published version (APA):

Patil, R. B. (2020). Prognostic and Prediction Modelling with Radiomics for Non-Small Cell Lung Cancer. [Doctoral Thesis, Maastricht University]. Maastricht University. https://doi.org/10.26481/dis.20201006rp

Document status and date:

Published: 01/01/2020

DOI:

10.26481/dis.20201006rp

Document Version:

Publisher's PDF, also known as Version of record

\section{Please check the document version of this publication:}

- A submitted manuscript is the version of the article upon submission and before peer-review. There can be important differences between the submitted version and the official published version of record.

People interested in the research are advised to contact the author for the final version of the publication, or visit the DOI to the publisher's website.

- The final author version and the galley proof are versions of the publication after peer review.

- The final published version features the final layout of the paper including the volume, issue and page numbers.

Link to publication

\footnotetext{
General rights rights.

- You may freely distribute the URL identifying the publication in the public portal. please follow below link for the End User Agreement:

www.umlib.nl/taverne-license

Take down policy

If you believe that this document breaches copyright please contact us at:

repository@maastrichtuniversity.nl

providing details and we will investigate your claim.
}

Copyright and moral rights for the publications made accessible in the public portal are retained by the authors and/or other copyright owners and it is a condition of accessing publications that users recognise and abide by the legal requirements associated with these

- Users may download and print one copy of any publication from the public portal for the purpose of private study or research.

- You may not further distribute the material or use it for any profit-making activity or commercial gain

If the publication is distributed under the terms of Article $25 \mathrm{fa}$ of the Dutch Copyright Act, indicated by the "Taverne" license above, 


\section{Prognostic and Prediction Modelling with Radiomics for Non-Small Cell Lung Cancer}

To obtain the degree of Doctor at Maastricht University, on the authority of the Rector Magnificus

Prof. dr. Rianne M. Letschert in accordance with the decision of the Board of Deans, to be defended in public on

Tuesday $6^{\text {th }}$ October 2020 at 10:00

by

Ravindra B Patil 


\section{Supervisor}

Prof. dr. ir. Andre Dekker

\section{Co-supervisor}

Dr. Leonard Wee

\section{Assessment Committee}

Prof. dr. D. De Ruysscher (Chair)

Prof. dr. W.J. Niessen (Erasmus University Rotterdam)

Dr. R. Monshouwer (Radboud University)

Dr. J. van Soest 


\section{Index}

Chapter 1

General Introduction and Outline of the Thesis

Chapter 2

Methodology in Prognostic and Prediction Modelling with Radiomics for Non-Small Cell Lung Cancer

Chapter 3 61

Deep Learning based Auto Contouring of Lung region

Chapter 4 81

Auto Delineation of Gross Tumor Volume in NSCLC

Chapter 5 .95

Does Radiomics Improves the Survival Prediction in Non-Small Cell Lung Cancer?

Chapter 6..................................................................................110

An Approach towards Automatic Classification of Tumor Histopathology of Non-Small Cell Lung Cancer Based on Radiomics Feature

Chapter 7 . .121

Fractal Analysis in Histology Classification of Non-Small Cell Lung Cancer

Chapter 8. .136

End to End Approach to Build Diagnostic Radiomics model 


\section{Index}

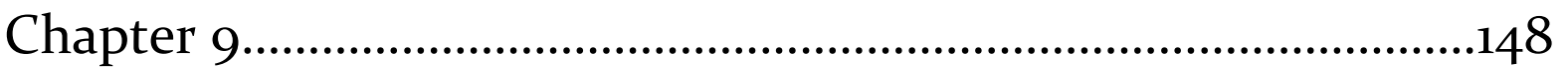
Discussion and Future Perspectives

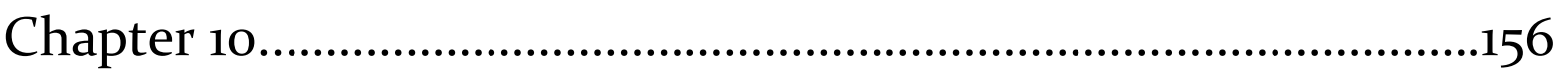
Summary

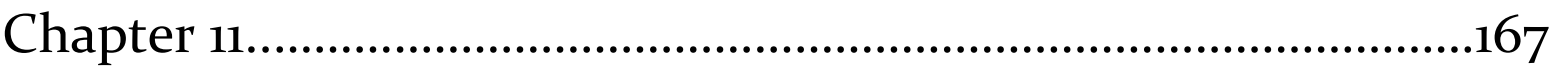
List of Publications 


\section{Chapter 1}

General Introduction and Outline of the Thesis

Ravindra Patil 
Lung cancer accounted for 1.7 million deaths worldwide in 2018 , which is also $20 \%$ of the cancer mortality as a whole [1]. Non-small cell lung (NSCLC) cancer alone accounts for $85 \%$ of all lung cancers making it the second most common cause of cancer in both men and women. Estimates by the American Cancer Society in the United States for 2016 place an incidence of 224,390 new cases of lung cancer (117,920 in men and 106,470 in women) and 158,080 deaths from lung cancer (85,920 in men and 72,160 in women). Every year, more people die of lung cancer than of colon, breast and prostate cancer combined [2].

While there has been clear and continuous progress being made in diagnosis and therapy for NSCLC, the focus for the future has begun to shift towards a personalized medicine paradigm. Personalized medicine is the tailoring of medical treatment to the individual characteristics of each patient [28]. It is hypothesized to have a better outcome for a patient population by exploiting individual differences in response to treatment, as compared to conventional population-based results applied across the entire population pool or a so-called “one size fits all” approach. Although this paradigm shift is scientifically interesting and clinically impactful, its practical implementation in healthcare is not devoid of major challenges.

Biopsies are presently considered to be the gold standard for determining the care plan in personalized lung cancer medicine. However, lung biopsies are not always possible and they carry significant risk of fatal complications. In addition, the results vary based on disease stage, biopsy timepoint and the what part of the tumor has been sampled for the biopsy [3]. Here is a clear and urgent need for a noninvasive approach to characterization of a whole tumour in vivo, to ultimately guide personalized medicine decisions towards better individual and population outcomes.

A noninvasive approach is addressed by shifting more focus towards medical imaging, which also plays a pivotal role in cancer diagnosis and staging. A central hypothesis of this thesis is that a more personalized approach could be feasible based on quantitative analysis of radiological images, specifically Computed Tomography $(\mathrm{CT})$, which represents a growing and actively evolving field known as "Radiomics".

The term "Radiomics" was coined by Lambin and other co-authors in a seminal publication [4]. It involves extracting numerous quantitative "features" from digital 
medical images and thereby converting these collections of individual points of intensity (that appear bright and dark to the unaided human eye) into numerical values that can be "data mined" through the assistance of high-performance machine learning algorithms. This minable data will be used to build descriptive and predictive models for a variety of applications such as histology classification, survival analysis and radiotherapy treatment planning.

One of the prominent areas of research activity in Radiomics is non-invasively linking imaging features (i.e numerical determinants of tumor phenotype) to the invisible tumor genotype. As shown by Lambin et al. [4], Radiomics provides a detailed quantification of imaging phenotype in terms of mathematical surrogates such as shape, texture, intensity heterogeneity etc. By linking such image-based features as markers of patient outcomes, Radiomics therefore provides a bridge from radiology imaging to personalized medicine, wherein the information provided by the image can be directly used to select a particular cancer treatment for an individual.

The present state and a parallel, complementary route including Radiomics supporting personalized cancer care is shown in Figure 1. Radiomics as a standalone tool is not sufficient, it needs to be augmented with other pertinent data and correlating these data sources with outcomes data, to produce accurate and robust evidence-based Clinical Decision Support System (CDSS). CDSS are computer programs that helps healthcare professionals in making a decision by linking clinical or/and health observations with accumulated facts and /or knowledge. In addition, computer-aided diagnosis (CAD) systems have been assisting the healthcare professionals for many years [4].

Radiomics may initially appear like an extension of computer-aided diagnosis and detection (CAD) systems, however there are noteworthy and significant differences. CAD systems are usually standalone systems that are designated by the Food and Drug Administration for use in either the detection or diagnosis of disease [4]. In CAD systems, outputs are typically binary in nature such as presence of lesion or not. However, Radiomics features can be used as variables in prognostic and predictive models, either for binary outcomes (where the results of the model are class probabilities of each possible outcome) or for survival analysis (where the result is a 
survival probability distribution). Radiomics therefore aids in hypothesis generation, hypothesis testing, or both.

Another major advantage of a Radiomics approach in lung cancer is that digital radiologic images are obtained for almost every patient with lung cancer and the images are acquired multiple times during treatment, as well as for post-treatment surveillance. All of these images are potentially sources of Radiomics data. Recent estimates indicate that up to 2314 exabytes (one exabyte = one billion gigabytes) [29] of imaging data will be produced in 2020, thereby placing Radiomics firmly in the "big medical data" domain.

In future, it can be envisaged possible that image interpretation for a vast majority of these imaging series could be augmented by using Radiomics, building an unprecedented source of big data that will expand the potential for discovering helpful correlations and evermore personalized and dynamic treatment approaches. 


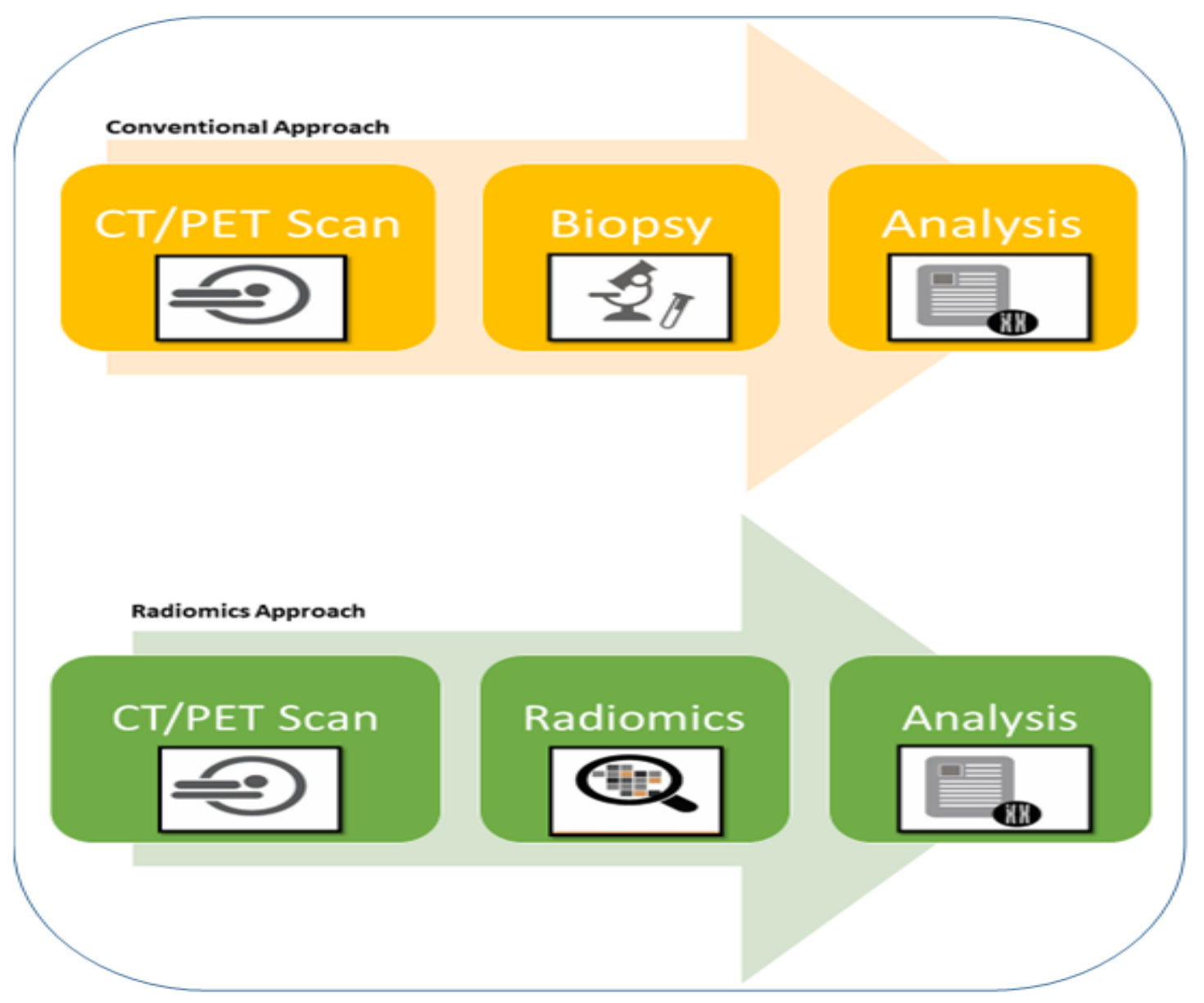

Figure 1. Conventional and Radiomics approach

\section{The Radiomics workflow}

The process of Radiomics involves acquisition of medical images, segmentation of the tumor regions, feature extraction, development of the model and validation using an external data set. The process is depicted in the Figure 2.

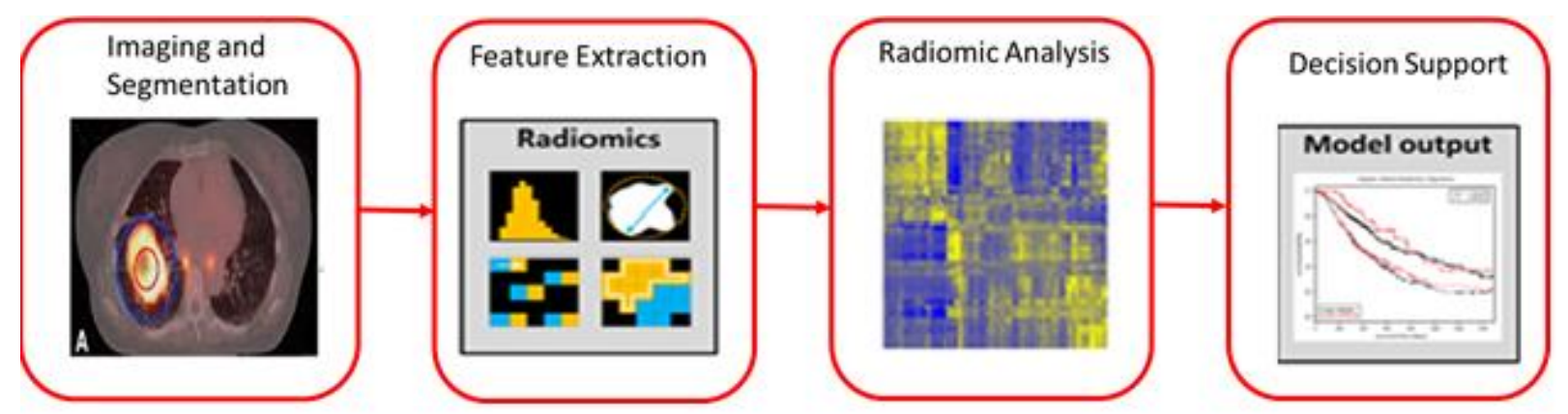

Figure 2. Radiomics work flow [images partly from (18) with permission] 


\section{Acquisition of Images}

The workflow begins with acquisition of high quality images from standard imaging systems such as CT, MR and PET with a standardized protocol. However, it is a known problem that medical image acquisition and reconstruction vary widely in clinical protocols; and standardization of these protocols across medical imaging centers is typically still an unsolved challenge. As a result, the absolute numbers derived from the images and thus derived Radiomics features might not be consistent across imaging sets. However, there has been significant effort put across by different entities such as the National Cancer Institute - Quantitative Imaging Network and the Quantitative Imaging Biomarkers Alliance (QIBA) of the Radiological Society of North America (RSNA) to arrive at uniform definitions for acquisitions and reconstruction [5 - 7].

\section{Volume identification and Segmentation}

Identification of the region(s) of interest in a medical image that contain the primary tumor and/or affected lymph nodes is a crucial step in Radiomics workflow. Other interesting regions other than the tumor itself include (i) the sub volumes of tumor known as habitats, which provide the information on blood flow, oedema, necrosis and cell density (ii) the peritumoral zones, which may contain information about growth, invasion and characteristics of the host substrate. In a radiological report it is often uncommon to find the descriptions about the sub volumes either due to the limitation in spatial resolution and/or contrast of the image provided by the acquisition system. It is also possible that some of this information is not considered by radiologist to be of clinical relevance, which suggests that a machine-aided human eye can uncover additional patterns that are indicative of clinical outcome. With Radiomics, these sub volumes can be easily captured for analysis. The underlying assumption is to gather as much data as possible from the radiological examination, and delegate to the future investigator to decide what data is of diagnostic/prognostic value for their respective clinical question. The concept of identifying the sub volumes that depict physiologically distinct regions is described in [8]. 
Segmentation separates volume regions relevant for analysis from the rest of the image. These regions of interest do not have well-defined boundaries and it is difficult to have a common segmentation approach that works for all medical images $[9,10]$. In addition, segmentation is carried out manually, semi-automatic, completely automatic or a combination of these leading to potential for bias across readers. However, the challenges that still exists in segmentation of the Region Of Interest (ROI) being ability to delineate ROI completely automatic across different scanner settings, vendor type and with different pathological conditions. This is one of the challenge that is addressed in this thesis by adapting Deep Learning techniques for segmentation, to achieve better generalization.

Further, in radiotherapy three kinds of regions are considered for tumor segmentation. The first is the position and extent of the macroscopic tumor, i.e. tumor tissue that can be seen, palpated or imaged; this is known as the gross tumor volume (GTV). The second volume contains the GTV plus a margin for sub-clinical i.e. unobservable disease spread; this is known as the clinical target volume (CTV). This is difficult because - by definition - it cannot be adequate seen without a microscope nor is it easy to objectively define for an individual patient. Future developments in imaging, especially towards the molecular level, are expected to allow more specific delineation of the CTV. The third volume, the planning target volume (PTV), accounts for uncertainties in patient positioning, planning or treatment delivery [11]. In case of Radiomics for this thesis, we only consider the GTV for all analysis, although Radiomics can be performed on any or all the above mentioned regions, and indeed even on nearby organs at risk of damage from the intervention. Manual delineation of GTV takes considerable amount of time by the radiation oncologist, there is an effort to address this challenge in this thesis to build automatic GTV detection and contouring algorithm such that there is significant reduction in the time spent for delineation. 


\section{Feature Extraction}

Extraction of prognostic information from a ROI is performed by means of feature extraction there by converting the pixels in a ROI into a minable feature set. The feature sets derived from the regions predominantly fall into Semantic or Agnostic groups. The semantic feature group encompasses a description of the ROI such as shape, location, and vascularity, whereas the agnostic feature set attempts to capture heterogeneity of tumor structure by computing first, second and higher order statistics. The first-order statistical features describe a distribution of image intensities within the ROI in terms of, for instance, mean, mode, maximum, skewness, kurtosis and minimum. Second order statistics provide measures of the textural information present inside the (ROI) and was first introduced in the year 1973 as Haralick (textural) metrics for satellite imagery [16]; these detail the heterogeneity of region and is computed by multiple methods such as gray level run length matrix, gray level co-occurrence matrix, and other closely-related matrix techniques as described in $[14,15]$. For higher order statistics, the image is first "enhanced" through the use of digital filter grids to the ROI to either sharpen contrast, reduce random noise, or enhance edges. For example, wavelet transformations are applied on image grids, which help decouple the original ROI into high and low frequencies of intensity modulation in the underlying ROI. Alternatively, if intention is to extract coarse texture pattern in the ROI, a Laplace transformation is applied. A family of spatial heterogeneity metrics, Minkowski functionals [15] can be used to describe patterns of intensity variation as a function of a sliding threshold value.

There are multiple studies in lung cancer, which have used different features to understand outcome in the area of diagnosis or therapy. A study by Kumar et al. used 202 features to designate CT images of lung cancer [17]. Another study by Aerts et al. [18] used 422 features by considering wavelet transforms and showed that radiomic features have prognostic power when used for patients with lung and head and neck cancer. There were two more studies with 662 and 522 features which used Radiomics by applying Laplace transform and fractal analysis [20, 21].However, there is still potential for new features to be explored other than the existing ones described above, that could be of aid in providing more diagnostic value. In this thesis, fractals have been explored in the search for image-derived markers that could aid in improving predictive 
performance of models. It is essential to point out at this point, that the methodologies developed in this thesis remain agnostic to the specifics of the Radiomics feature types being used, and indeed it is common to combine different classes of features together to develop a model.

The number of features from a region of interest in Radiomics varies from 200 to 1000 or even more, making this a complex modelling task. Due to the large number of these features, together with automated methods of feature selection to "tune" for the best performing model, there is a high risk of overfitting to the model development data as well as a high risk of falsely positive discoveries generally [30]. As a rule of thumb the ratio of the number of features to the number of outcome events should be kept small as possible. In order to avoid overfitting of data due to a large number of features, redundant features (e.g. highly correlated or uninformative features) should be eliminated. One approach is to do test - retest to concur on the reproducibility of the results, if the data is available [18]. In addition, there are multiple methods within wrapper and embedded selection approaches been used to fine-tune the features list. Although, some of the work has been performed to overcome the challenge of overfitting. However, still the challenge of overfitting do exist during building of prognostic and predictive modelling. This challenge has been analyzed in this thesis by identifying which of the feature selection techniques are employed, in particular to cancer care and it is addressed by proposing the approaches that could be adapted when building the prognostic and predictive modelling.

\section{Model development}

The next step following the mining of quantifiable data from the images is to build the model that helps in predicting various events such as the diagnosis of tumor phenotypes, survival, histopathology and treatment planning. There are two approaches for this i.e data driven and hypothesis based models.

In the case of data driven models, all the features extracted begin with equal priority and hence no prior knowledge regarding the predictive potential of the feature 
set is assumed. The underlying assumption is that all the features contribute equally and independently towards the event outcome, although it is feasible to employ feature selection techniques to select most significant features towards the endpoint. In this approach both supervised machine learning algorithms (Support Vector Machines, Logistic Regression, Random Forests, Neural Nets etc. ) and unsupervised learning with aid of clustering techniques are used to build the model [22-24]. In contrast, within a hypothesis driven modeling approach an expert clinician or domain expert grades the features based on the prior experience of the clinical outcomes.

There is no clear distinction on which approach is best for building the model, as the answer varies based on the type of covariates used, event outcome and the data. At the very minimum, the Radiomics model should be compared with other perhaps simpler models, to establish whether the Radiomics analysis adds value to the clinical question being explored. The hypothesis being that the model, which takes into account not only the Radiomics features but also patient information and the clinical profile such as (histology, dose, treatment time, mutation) into account, is better at predicting the outcome [25].

However, the challenges that exists in the model development being the ability to handle sparse data as there is a high possibility there might be missing information due to unavailability of records or missing to record the observation. Another, being the amount of perturbation in output due to change in the dataset that the model can handle and the need of model to have a clearly defined endpoint, such as identification of histology or survival time of the patient etc. These challenges are studied in this thesis and approaches have been suggested on how the clinical models can over these challenges by adapting different techniques.

\section{Model Validation}

The metric for evaluation of the model varies based on whether its discriminative model or calibration based. The discriminative models are measured in terms of area under the curve (AUC) and the calibration models uses goodness-of-fit estimates to arrive at 
the model performance $[25,26]$. In addition, there are multiple other evaluation measures that can be adapted such as Gini Coefficient, Log loss, Kolomogorov Smirnov chart, Concordant - Discordant ratio based on the type of the model being developed [27]. However, the challenges of model validation in cancer care ranges from lack of independent data for validation to not employing robust validation criteria for internal and external validation. In this thesis, review of different models built, in particular for NSCLC, has been analyzed and have highlighted the merits and demerits of each of the methodology that are employed in validating the models. In addition, suggestions have been provided on how the model validation needs to be performed to remove bias as well as improve the clinical applicability and deployment.

\section{Objectives and outline of the thesis}

In this thesis, we tried to address the above mentioned challenges and enhance the application of Radiomics by conducting different Radiomic analyses on large cohorts of NSCLC patients.

This thesis covers the approach adapted in building the predictive models for NSCLC and the usage of machine learning and deep learning models to enhance the accuracy and automation of building end to end clinical decision support systems that aims at improving the cancer care and in particular for NSCLC.

Chapter 1 provided the general introduction of Radiomics including the workflow, applications, and associated challenges. In addition, it briefly outlined the organization of the thesis.

Chapter-2 provides the details on different methodologies for prognostic and prediction modelling with Radiomics for Non-Small Cell Lung Cancer. This chapters discusses challenges and the drawbacks associated with current approaches and the recommendation on the how to build the high-quality prognostic and predictive models. 
Chapter-3 presents one of the steps in end-to-end automation of building a CDSS. This chapter describes a Deep Learning based approach to segment the region of the lung in the given CT volume without any seed point initialization. It highlights the effectiveness of Deep Learning approaches over the traditional segmentation approaches.

Chapter 4 describes an approach of auto extraction of the Gross Tumor Volume from a given CT volume using a deep learning model, which aids in significant reduction of the time that is required for manual contouring. This chapter also highlights the need of autoconturing and shows the efficacy of deep learning models.

Chapter 5 tries to address the question "Does Radiomics improve the survival prediction in non-small cell lung cancer?" The chapter concludes with the comparison of a survival model using the existing approach to that of a model that includes radiomic features and the results suggests that Radiomics helps in improving the survival prediction in NSCLC.

Chapter 6 describes a Radiomics study to extract the histopathology subtype from image data. This study has shown that the additional Radiomics information improves the accuracy of the detection of the histology of non-small lung cancers by $20 \%$ as compared to the traditional approach of just considering volumetric and shape based features.

Chapter 7 addresses the need of fractals as new features complementary to existing Radiomics features. The aggressiveness of the tumor spread can be assessed from fractal characteristics. Fractals are mathematical method for quantification of irregular patterns. This chapter concludes by demonstrating the role of fractal dimensions in improving the histology classification of NSCLC by $8 \%$. 
Chapter 8 discusses the approaches to build End to End diagnostic Radiomics model for clinical application and also provides insights on the need of using machine learning and deep learning tools.

Chapter 9 provides a general discussion of the results presented in this thesis and related future perspectives.

\section{References}

[1] Freddie Bray et.al. "Global Cancer Statistics 2018: GLOBOCAN Estimates of Incidence and Mortality Worldwide for 36 Cancers in 185 Countries", CA CANCER J CLIN 2018; o:1-31. doi: 10.3322/caac.21492.

[2] A report on Lung Cancer (Non-Small Cell), American Cancer Society, 2016 (http://www.cancer.org/acs/groups/cid/documents/webcontent/oo3115-pdf.pdf).

[3] Wu C, Maher MM, Shepard JA. Complications of CT-guided percutaneous needle biopsy of the chest: prevention and management. AJR Am J Roentgenol. 2011 Jun;196(6):W67882. doi: 10.2214/AJR.10.4659.

[4] Lambin P, Rios-Velazquez E, Leijenaar R, et al. Radiomics: extracting more information from medical images using advanced feature analysis. Eur J Cancer 2012;48:441-6.

[5] Institute of Medicine I. Evolution of Translational Omics. Lessons learned and the path forward. Washington, DC: National Academies Press, 2012.

[6] Clarke LP, Sriram RD, Schilling LB. Imaging as a Biomarker: Standards for Change Measurements in Therapy workshop summary. Acad Radiol 2008;15(4):501-530.

[7] Shankar LK, Hoffman JM, Bacharach S, et al. Consensus recommendations for the use of 18 F-FDG PET as an indicator of therapeutic response in patients in National Cancer Institute Trials. J Nucl Med 2006;47(6):1059- 1066.

[8] Clarke LP, Nordstrom RJ, Zhang H, et al. The Quantitative Imaging Network: NCI's Historical Perspective and Planned Goals. Transl Oncol 2014;7(1):1-4.

[9] Buckler AJ, Bresolin L, Dunnick NR, Sullivan DC; for the Group. A collaborative enterprise for multi-stakeholder participation in the advancement of quantitative imaging. Radiology 2011;258(3):906-914.

[10] Gatenby RA, Grove O, Gillies RJ. Quantitative imaging in cancer evolution and ecology. Radiology 2013;269(1):8-15.

[11] Velazquez ER, Parmar C, Jermoumi M, et al. Volumetric CT-based segmentation of NSCLC using 3D-Slicer. Sci Rep 2013;3:3529.

[12] Hatt M, Cheze-le Rest C, van Baardwijk A, et al. Impact of tumor size and tracer uptake heterogeneity in (18) F-FDG PET and CT non-small cell lung cancer tumor delineation. J Nucl Med 2011;52:1690-7.

[13] Neil G Burnet,* Simon J Thomas, $\ddagger$ Kate E Burton, $†$ and Sarah J Jefferies†, Cancer Imaging. 2004; 4(2): 153-161. Published online 2004 Oct 21. doi: 10.1102/14707330.2004 .0054

PMCID: PMC1434601. Defining the tumour and target volumes for radiotherapy 
[14] Davnall F, Yip CS, Ljungqvist G, et al. Assessment of tumor heterogeneity: an emerging imaging tool for clinical practice? Insights Imaging 2012;3(6):573-589.

[15] O'Connor JP, Rose CJ, Waterton JC, Carano RA, Parker GJ, Jackson A. Imaging intratumor heterogeneity: role in therapy response, resistance, and clinical outcome. Clin Cancer Res 2015;21(2):249-257.

[16] Haralick RM, Shanmugam K, Dinstein I. Textural features for image classification. IEEE Trans Syst Man Cybern 1973;SMC- 3(6):610-621.

[17] Kumar V, Gu Y, Basu S, et al. Radiomics:vthe process and the challenges. Magn ResonvImaging 2012;30(9):1234-1248.

[18] Aerts HJ, Velazquez ER, Leijenaar RT, et al. Decoding tumour phenotype by noninvasive imaging using a quantitative Radiomics approach. Nat Commun 2014;5:4006.

[19] Ravindra Patil, Geetha Mahadevaiahı, and Andre Dekker. An Approach Toward Automatic Classification of Tumor Histopathology of Non-Small Cell Lung Cancer Based on Radiomic Features. TOMOGRAPHY, December 2016, Volume 2, Issue 4: 374377 DOI: 10.18383/j.tom.2016.00244

[20] Grossmann P, Grove O, El-Hachem N, et al. Identification of molecular phenotypes in lung cancer by integrating Radiomics and genomics. Sci Transl Med (in press).

[21] Pickup L, Talwar A, Stalin S, et al. Lung nodule classification using learnt texture features on a single patient population [abstr]. In: Radiological Society of North America Scientific Assembly and Annual Meeting Program. Oak Brook, Ill: Radiological Society of North America, 2015; SSMo6.

[22] Aerts, H. et al. Identification of residual metabolic-active areas within NSCLC tumours using a pre radiotherapy FDG-PET-CT scan: a prospective validation. Lung. Cancer. 75, 73-76 (2012).

[23] Aerts, $\mathrm{H}$. et al. Identification of residual metabolic-active areas within individual NSCLC tumours using a pre-radiotherapy (18)Fluorodeoxyglucose-PET-CT scan. Radiother. Oncol. 91, 386-92. (2009).

[24] Carrasco, J.L., King, T.S. \& Chinchilli, V.M. The concordance correlation coefficient for repeated measures estimated by variance components. J Biopharm Stat 19, 90-105 (2009).

[25] Carrasco, J.L., Phillips, B.R., Puig-Martinez, J., King, T.S. \& Chinchilli, V.M. Estimation of the concordance correlation coefficient for repeated measures using SAS and R. Comput Methods Programs Biomed 109, 293-304 (2013).

[26] Weimiao Wu, Chintan Parmar,Patrick Grossmann, John Quackenbush, Philippe Lambin,4 Johan Bussink,6 Raymond Mak and Hugo J. W. L. Aerts. Exploratory Study to Identify Radiomics Classifiers for Lung Cancer Histology

[27] Lin, L.I. A concordance correlation coefficient to evaluate reproducibility. Biometrics 45, 255-68 (1989).

[28] Vogenberg FR, Isaacson Barash C, Pursel M. Personalized medicine: part 1: evolution and development into theranostics. P T. 2010;35(10):560-576.

[29] Aiello M, Cavaliere C, D'Albore A, Salvatore M. The Challenges of Diagnostic Imaging in the Era of Big Data. J Clin Med. 2019;8(3):316. Published 2019 Mar 6. doi:10.3390/jcm8030316

[30] Chalkidou A, O'Doherty MJ, Marsden PK (2015) False Discovery Rates in PET and CT Studies with Texture Features: A Systematic Review. PLoS ONE 10(5): eo124165. https://doi.org/10.1371/journal.pone.0124165 


\section{Chapter 2}

\section{Methodology in Prognostic and Prediction Modelling with Radiomics for Non-Small Cell Lung Cancer}

Ravindra Patil, Leonard Wee, Andre Dekker

Accepted for International Journal of Precision Oncology 


\section{Abstract}

OBJECTIVE: The aim of this study was to systematically review the literature to synthesize and investigate repeatability and reproducibility of radiomic features, considered in building prognostic and prediction modelling with respect to Non Small Cell Lung Cancer (NSCLC).

METHODS: The PubMed database was searched using combinations of the broad Haines and Ingui filters along with a set of text words specific to cancer, Radiomics, reproducibility and repeatability. This systematic review was performed by two reviewers working entirely independently, and has been reported in compliance with PRISMA guidelines.

RESULTS: Out of 624 unique records, 41 full text articles were subjected to review. The studies were primarily in NSCLC. The imaging modalities were CT, PET and cone-beam CT - no studies addressed MR. Only 7 studies addressed in detail every methodological aspect related to image acquisition, pre-processing and feature extraction. Only a few studies have made either the image set or software, or both, openly accessible. Due to the heterogeneity in statistical metrics, a meta-analysis of pooled data was not possible. CONCLUSIONS: The repeatability and reproducibility of radiomic features are sensitive in varying degrees to processing details such as image acquisition settings, image reconstruction algorithm, image pre-processing and software used to extract radiomic features.

KEY POINTS: Intra-class and concordance correlations were the most widely used statistical metrics, but cut-offs were selected arbitrarily and were variable. First-order features were overall more reproducible than shape metrics and textural features. Entropy was consistently reported as one of the most stable first-order features. There was no emergent consensus regarding either shape metrics or textural features.

Key words : Radiomic Features, Modelling, NSCLC 


\subsection{Introduction}

Lung cancer remains a major public health issue in developed countries, accounting for about $20 \%$ and $28 \%$ of cancer-related deaths in Europe and the United States of America, respectively [1]. In 2012, the World Health Organization estimated in excess of 1.2 million new cases of lung cancer and 1 million deaths from lung cancer globally. More people die of lung cancer than of colon, breast and prostate cancer combined [2]. Nonsmall cell lung (NSCLC) cancer accounts for $85 \%$ of all the lung cancers, making it the second most common type of cancer in both men and women. While a range of intervention options exists for NSCLC, a significant challenge remains for accurate risk stratification and optimal treatment selection.

Recent interest in personalized medicine is driven by an expectation of better outcomes [3] if unique characteristics of a person and their disease can be taken into account. There is a wide range of potential prognostic and/or predictive factors [4], among which are image-derived markers, i.e. "Radiomics". The aim is to identify factors that accurately indicate histology, intra-tumor heterogeneity and differential response to treatments. Radiomics-assisted personalized medicine requires computerized extraction of quantitative image metrics, i.e. "features", from vast volumes of medical imaging examinations that can subsequently be linked to compare outcomes of different diseases/treatments. The hypothesis is that radiographic images contain more information about the tumor phenotype than human-generated semantic features derived by the unaided eye. Radiomics analysis may be readily integrated into automated multi-factorial decision support tools [4.a], that could assist physicians in selecting a superior treatment for a given individual.

\section{Radiomics as disease characterization}

A Radiomics-guided approach has been investigated as a non-invasive form of tissue biopsy [4.b]. Biopsies are the gold standard for determining histology and differentiation grade, but they are also associated with some risk of complications, such as pneumothorax [5]. In lung cancer, fine needle biopsies take selective samples, thus results may vary due to finite sampling and tumor heterogeneity [6]. 
Figure 1 depicts a hypothetical workflow incorporating radiological imaging, and shows how Radiomics could play a role in pre-treatment disease characterization. Small biopsies and Radiomics analysis could complementarily inform tumor characterization and individual prognosis, which is then taken into account when selecting treatment. A Radiomics-guided analysis has an advantage because it attempts to characterize the entire tumor in situ and can be repeated at multiple time points.

\section{Radiomics as prognostic or predictive marker}

Several key publications are credited with showing how radiomic features may contain additional information pertaining to treatment outcome and/or response monitoring $[46,44,21]$. As shown in Figure 1, complementary information could be obtained from radiological images and post-operative tissue samples to predict treatment outcome. For (chemo)-radiotherapy treatment, radiomic features from follow-up imaging could be used to non-invasively monitor tumor response.

\section{Distinction from previous review(s)}

Generalizability and methodological questions pertaining to Radiomics-based models are active research topics. Potential pitfalls have been identified in several other general reviews [20, 61, 62, 63]. Specifically, methodological steps in congruence with TRIPOD [56] need to be taken in the reporting of Radiomics modelling studies with many candidate features (sometimes well in excess of 100 features per outcome) to mitigate risks of over-fitting, false-positive associations and narrow clinical generalizability of models using external independent validation. A recent systematic review addressing studies of repeatability and reproducibility of Radiomics features indicates that lack of standardization of feature definitions, clarity of reporting and open access (to images and software) presently slows down efforts to generalize and apply Radiomics in clinical situations [64]. 


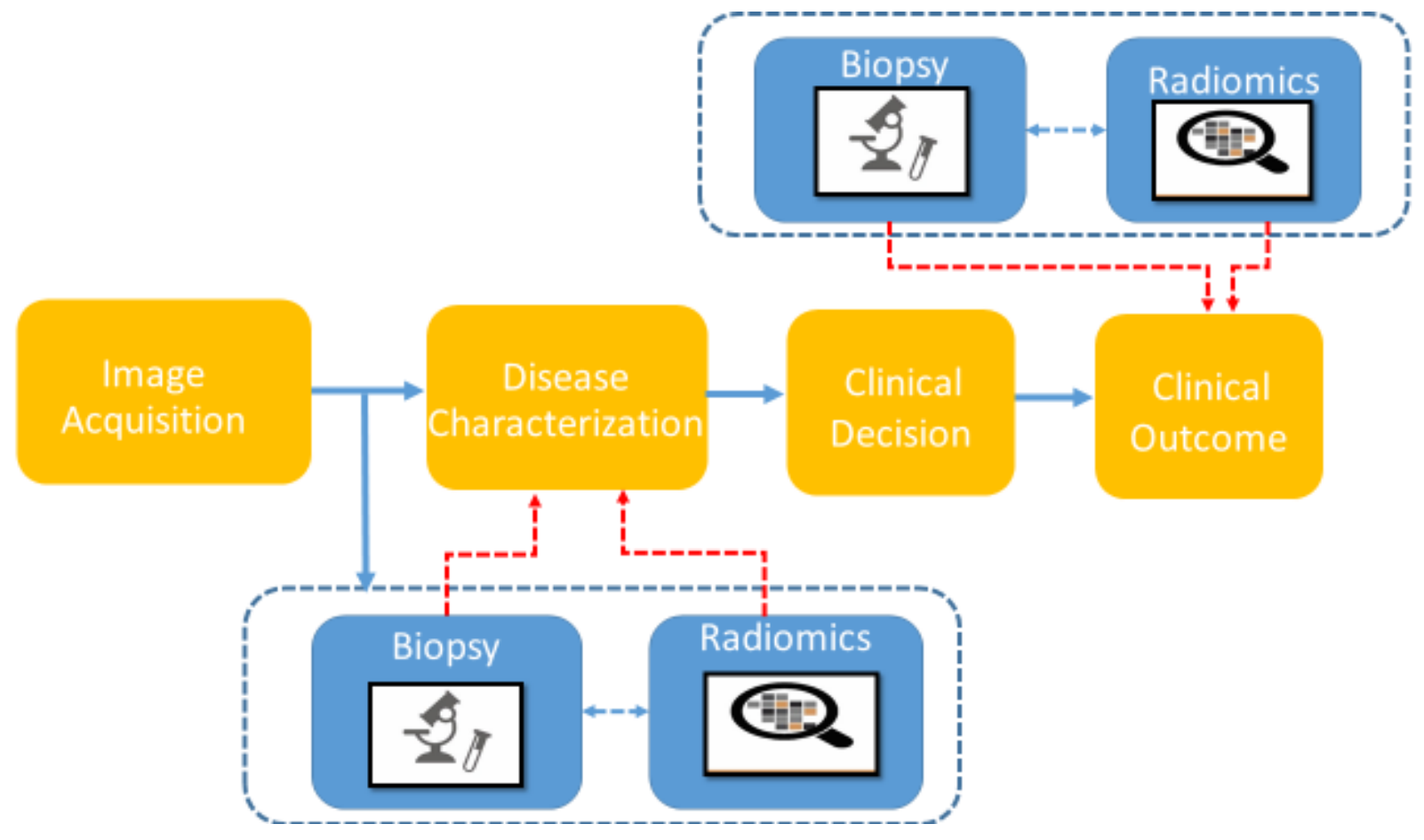

Figure 1. Generic clinical workflow incorporating radiological examinations and biopsies, showing the potential applications of Radiomics-based analysis for disease characterization, treatment selection and follow-up.

\section{$\operatorname{Aim}(s)$ of this review}

In this review, we focus on updates in methodological robustness that have come to light since the previous reviews. Further, we wish to understand which are the prominent features that are considered by the studies to build predictive and prognostics models. In addition, we aim to analyze the approaches considered for feature selection, the software packages used for feature extraction and the methodology adapted to perform the validation while also identify the shortcomings and strengths associated with the studies.

\section{Overview of Radiomics model building and model validation}

A generic Radiomics procedure for cancer outcome prediction comprises two principal steps; (i) feature extraction and (ii) signature computation. Feature extraction refers to an automated process of calculating quantitative image metrics from a radiological 
image annotated with a region of interest (ROI), typically the gross tumor volume (GTV). The ROI may be drawn via a manual, semi-automatic or automatic process, generally known as "segmentation". The image and ROI are paired inputs into specialized computer software that calculates radiomic features.

A signature is a combination of one or more radiomic features that is statistically associated with the odds of a given clinical outcome. A prognostic/predictive signature may comprise either clinical observables or radiomic features, or a combination of both types. During model development, clinical risk factors (e.g. disease stage) can be combined with Radiomics features in order to fit a statistical model to the observed outcomes (Fig. 2a). In validation and prior to clinical use, the radiomic features and/or clinical risk factors are used as to predict the expected outcome using an already-fitted model (Fig. 2b) and compared to actual outcome.

In general, the data used to validate the model should be entirely independent from the data used to build the model, and not used anywhere during the model development process. This is known as external validation. If a subset of data has been used for interim validation of a model-under-development, this is known as cross-validation. Suitable external validation data must be different from the model-building data in at least one way, for instance, collected over a different time period, or at a different hospital/institution, or in a different clinical practice setting or in an intentionally different medical indication. 


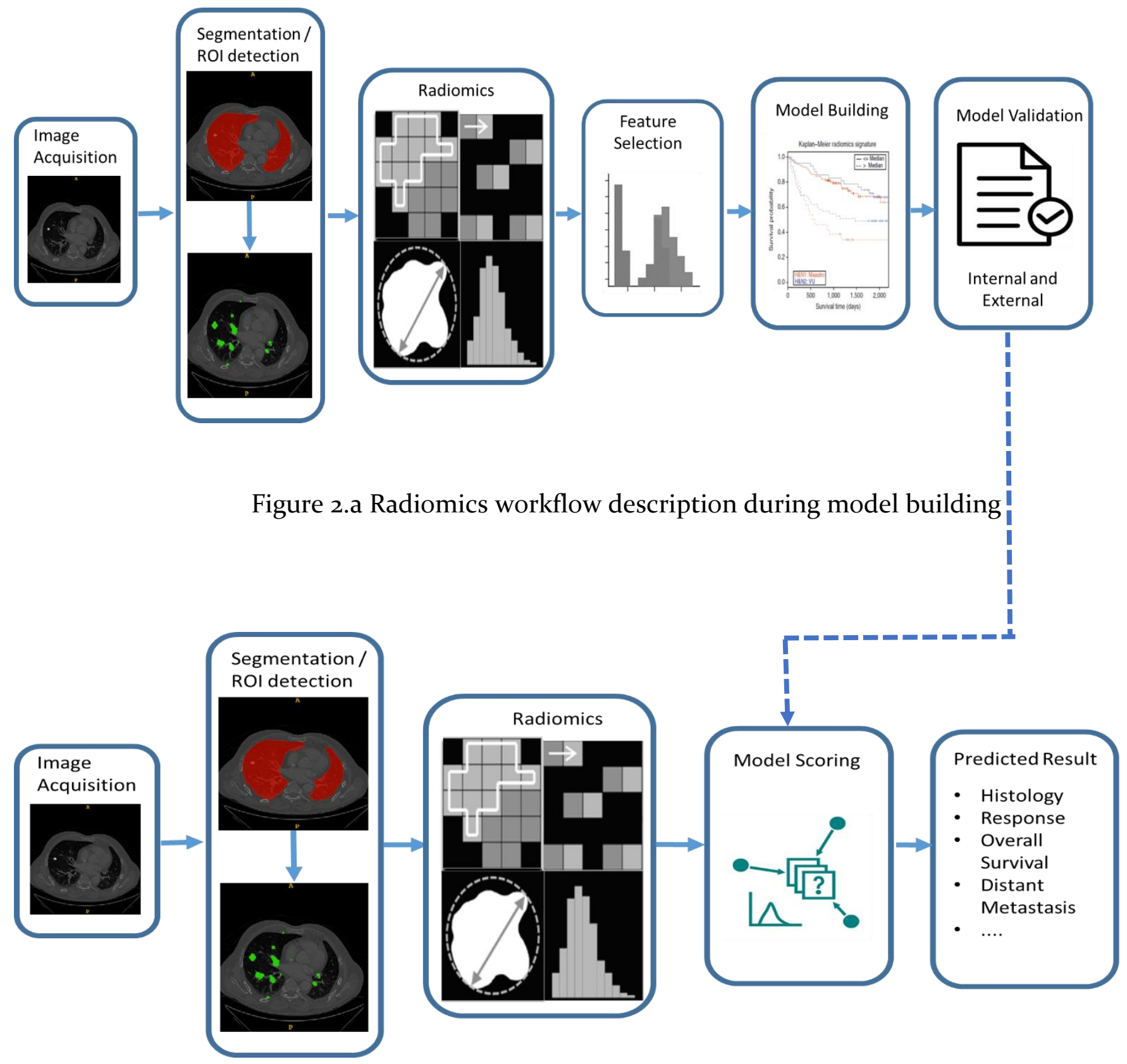

Figure 2.b Radiomics workflow description during model scoring 


\section{Imaging modalities}

In Radiomics studies of NSCLC, the most commonly encountered tomographic images are obtained from CT (Computed Tomography) or PET (Positron Emission Tomography), employing a wide variety of imaging protocols. There are vast numbers of permutations of image acquisition parameters, therefore repeatability and reproducibility of radiomic features needs to be considered when developing and validating models [64]. To date, radiomic features are modality specific, though it is possible that a radiomic signature might comprise features derived from different imaging modalities. Standardization of image acquisition for the purpose of generalizing Radiomics across multiple institutions remains an open question and may be hampered by institutional preferences. There are ongoing efforts by the National Cancer Institute Quantitative Imaging Network (NCI-QIN) and the Quantitative Imaging Biomarkers Alliance (QIBA) of the Radiological Society of North America (RSNA) to arrive at some consensus for standards [8-10].

\section{Segmentation}

Defining regions of interest (ROIs) within a radiological examination is a crucial step in radiomic analysis. The interesting part of the image needs to be segregated from the rest, in order to isolate potentially useful information. Specifically in NSCLC, ROIs of relevance are the hyperintense tumors in the lung. These may be delineated as a single aggregated ROI with the index tumor or as individual ROIs, but it is important to clearly document the ROI from which the radiomic features are derived. Besides the tumor and nodes, it is also possible to consider radiomic features derived from adjacent areas in healthy tissue, as these may encode some information about blood flow, oedema, necrosis, cell density or other potentially useful risk factors [11].

Besides manual segmentation, there are various (semi-)automated segmentation algorithms that may be used to define a ROI, that typically fall into one of the following categories: (i) intensity-based, (ii) object shape-based or model-based, (iii) neighboringanatomy constrained, (iv) region-growing based, or (v) artificial neural network (ANN) based. Each of these vary in degree of automation and algorithmic accuracy. Shape- 
based and ANN-based models have shown more consistently reproducible results, but it is an open question about what is an adequately accurate ROI for Radiomics. It is rare to find a single tumor segmentation algorithm that works on all medical images [13, 14]. Related to this is that inter-physician differences in manual ROI annotation is also a major source of disagreement [15]. A study [86] suggests that machine-assisted segmentation (automatic initial segmentation, followed by physician review and manual corrections) provides both more consistent and more accurate ROIs of NSCLC than manual segmentation alone.

\section{Feature Extraction}

Each radiomic feature falls into one of two groups - Semantic Features or Agnostic Features. Semantic features generally encompass descriptive metrics of an ROI such as volume, location, sphericity, longest dimension, etc. Thus, semantic features are likely closest to what human radiologists report. Agnostic features relate to arbitrary metrics such as describing spatial variation in image intensity.

First-order agnostic features quantify only the numerical distribution of intensities, e.g. the mean. Second-order features quantify spatial variation (i.e. texture), and were first introduced in 1973 [16]. Examples of second-order features include gray level run-length, gray level co-occurrence and other $(2 \mathrm{D}$ or $3 \mathrm{D})$ matrix measures as described in elsewhere $[17,18]$. Third- and higher order features require the application of digital image filters immediately prior to feature extraction. The primary purpose of digital filtering is to amplify spatial patterns. For example, directional wavelets (akin to Fourier transformations in analog signal processing), resampled to isotropic spacing, preferentially select intensity non-uniformity of either high or low modulation frequency. A Laplacian-of-Gaussian (LoG) filter may be used to emphasize coarse textural patterns. A hybrid class of features has been investigated that combines information from intensity, morphology and texture, such as threshold Minkowski functional analysis [19] and fractal analysis [65]. 
Radiomics features derived after resampling and filtering tend to depend on how the methods are implemented and this contributes to discrepancies between Radiomics software packages.

\section{Feature selection}

An important procedure step is to select features during model development, and we have included this in Figure 2 a before model-building. Selection may be achieved by stepwise regression, recursive feature elimination or a regularized machine learning approach (such as ElasticNet). The reader is referred to a recent methodological review of machine-based classifiers for cancer outcomes modelling [66]. A human expert may also hand-pick features that are known to be less sensitive to image acquisition settings and digital pre-processing. Finally, unsupervised learning methods (such as hierarchical clustering) may be used to reduce the number of features in the final model [25-27].

\section{Model development and validation}

Regarding model development and validation, two main approaches are adopted in Radiomics-based modelling; a purely data-driven approach versus a hypothesis-based approach. The data-driven approach assumes no a priori knowledge. A vast number of Radiomics features (of the order of $10^{2}$ or greater) is calculated from each ROI. A smaller set of features contributing to the final model is generated during the model development.

A hypothesis-driven approach requires that a clinical domain expert initially selects a candidate set of features to be examined during model development. Such prior knowledge may come from independent studies of the association between these candidate features to either an outcome or a biological phenomenon of interest.

There is a justified concern that model parameter-tuning based on a large number of Radiomics features on relatively few outcome events will lead to high risk of false positive association and over-fitting [67]. Some mitigation measures such as multiplefold cross-validation and repeated subsampling are generally accepted as best practice. 
Even so, it is broadly accepted that a lack of external validation leads to overestimation of model performance.

A pragmatic approach to Radiomics model development therefore requires some combination of data and hypothesis driven approaches, together with robust feature selection and external validation.

\subsection{Methods}

We performed the systematic review and analysis until October 2018. The included articles met all of the eligibility criteria given below.

\section{Report design}

We accepted only peer-reviewed full-text reports published in journals that presented results of prognostic or predictive statistical models based on the use of one or more Radiomics features. Reports giving purely qualitative results, reports published as letters to the editor, or in the form of abstracts (such as conference proceedings) were not included.

\section{Language}

Only full-text reports in the English language were included in this review.

\section{Information sources}

A manual electronic search was conducted in PubMed (MEDLINE citations had been previously merged into the PubMed repository). No search was made in grey literature sources for unpublished studies or conference proceedings.

\section{Search Strategy}

A search of PubMed citations was performed using a broad Haynes [68] and Inqui [69] filters in combination with the modifications proposed by Geersing et al. [70] (each criteria combined using an 'OR' logical operator). A preliminary search in January 2017 for histological prediction using a Radiomics approach was combined with the results of the above in October 2018. For the final search, additional filters for "non small cell 
lung cancer" (MeSH major topic), and text (abstract/title) strings "Radiomics", "quantitative imaging”, "features” and “texture/textural analysis” were applied.

\section{Eligibility criteria}

We allowed studies specifically using radiomic features in a multivariable statistical model for either histology or treatment outcomes for NSCLC. Treatment of metastatic diseases from primary NSCLC was allowed, as were any interventions involving surgery, chemotherapy, radiotherapy and combinations thereof.

We restricted the date range of reviewed articles from January 2011 to October 2018, as clinical interventions for NSCLC and mathematical techniques had changed significantly since earlier investigations. Due to potential bias in very small sample sizes, we restricted our review to studies with more than 10 human subjects.

\section{Data Management}

Electronic full text articles were downloaded and a review-specific shared DropBox folder was set up to handle document collection, data extraction forms as well as disseminating the reviewer findings.

\section{Selection Process}

Two reviewers (RP and LW) worked jointly throughout all phases of the study selection process (abstract screening, eligibility and inclusion for full-text evaluation). The reviewers jointly compared the titles and the abstracts against the inclusion criteria. Discrepancies were resolved by discussion until unanimous agreement.

\section{Data Extraction}

From each reviewed paper, we extracted information about sample size, screening/treatment type and imaging modality. For histological prediction, we considered whether the study involved histology classification at the human subject level, or nodule classification at the level of each examined nodule. For interventional procedures, we looked at the overall staging of the disease prior to treatment. 
Among the intervention studies, we extracted which type of features were reported to be predictive. We also recorded what software was used to calculate radiomic features and what kind of feature selection (if any) had been used during model construction. We extracted the number of features used during model building, the number of events (for the outcomes of interest), the reported predictive performance of the model, and whether there had been an external validation of the model.

\section{Population}

The population reported in the study are subjects diagnosed with non-small cell lung cancer. Animal subjects, biological samples outside of the human body, and non-clinical imaging studies were specifically excluded. Eligible results may have come from either retrospective or prospective studies, and may have been either observational or experimental in nature. Both single and multi-institution studies were allowed.

\section{Outcomes for synthesis}

We specifically assessed the following outcomes: survival after treatment, histology classification, pathological response and treatment failure (such as progression, recurrence or metastases).

\subsection{Results}

\section{Radiomics for predicting histology or cancer stage}

Our literature survey located the same two studies Dhara et al, Dilger et al $[35,36]$ on NSCLC nodule classification, as found by Scrivener et al. in 2016 [61]. Both studies used nodules as the primary unit of analysis. Further, we located three new studies of Radiomics for either staging or nodule classification [3, 22, 31] after Parmar et al. [34]. Characteristics of the studies are summarized in Table 1. All six studies used CT as the imaging modality. Parmar et al [34] included a mixture of lung and head and neck 
cancers, $\mathrm{Wu}$ et al. [31] included other lung cancers and Andersen et al. [37] included cases with suspected but not confirmed lung cancer.

\begin{tabular}{|c|c|c|c|c|}
\hline $\begin{array}{c}\text { Study Ref. } \\
\text { (Author:Yea } \\
\text { r) }\end{array}$ & $\begin{array}{c}\text { Sample } \\
\text { Size }\end{array}$ & Screening Subjects & Classification & Imaging \\
\hline Dhara:2016 & $891^{*}$ & Lung cancer & $\begin{array}{c}\text { Nodule } \\
\text { classification }\end{array}$ & $\mathrm{CT}$ \\
\hline Dilger:2015 & $50^{*}$ & Lung cancer & $\begin{array}{c}\text { Nodule } \\
\text { classification }\end{array}$ & $\mathrm{CT}$ \\
\hline $\begin{array}{l}\text { Andersen:201 } \\
6\end{array}$ & 29 & $\begin{array}{c}\text { Suspected } \\
\text { malignancy in } \\
\text { mediastinal nodes }\end{array}$ & Histology type & $\mathrm{CT}$ \\
\hline $\begin{array}{l}\text { Desseroit:201 } \\
6\end{array}$ & 116 & $\begin{array}{l}\text { Non-small cell lung } \\
\text { cancer }\end{array}$ & Histology type & $\mathrm{PET} / \mathrm{CT}$ \\
\hline Parmar:2015 & 878 & $\begin{array}{l}\text { Lung and } \mathrm{H} \& \mathrm{~N} \\
\text { cancer }\end{array}$ & Histology type & $\mathrm{CT}$ \\
\hline Patil:2016 & 322 & $\begin{array}{c}\text { Non-small cell lung } \\
\text { cancer }\end{array}$ & Histology type & $\mathrm{CT}$ \\
\hline Wu:2016 & 350 & Lung cancer & Histology type & $\mathrm{CT}$ \\
\hline
\end{tabular}

Table 1. The asterisk $\left(^{*}\right)$ denotes the sample size in number of nodules. For all others, the sample size is the number of patients. Abbreviation for imaging modality: (CT) computed x-ray tomography, 
Dhara et al. [35] reported an AUC ranging from 0.841 - 0.951 for three different configurations used for binning the nodules based on different combinations of the rank of malignancy. The model was built using 891 unique nodules, however there was no explanation of how the subjects were selected for analysis, leading to a potential for selection bias. There was missing documentation about acquisition parameters and no external validation in an independent dataset, therefore we would expect that the reported AUCs would be over-optimistic.

Dilger et al. [36] introduced features of the lung parenchyma and showed a modest improvement of nodule classification (AUC increased from 0.918 to 0.938). This model is at risk of over-fitting, since only 50 nodules were considered in their analysis and they have selected in total 58 features for the model building. There was no external validation of model performance. Andersen et al. [37] used Radiomics to differentiate malignant and non-malignant mediastinal nodes in 29 patients with NSCLC with a reported AUC of 0.834 . To enhance the radiomic features of the lymph nodes, they applied a Laplacian of Gaussian filter within the ROI. The authors claimed that insample reproducibility was excellent, but did not demonstrate this in an external validation dataset with nominally similar image acquisition settings.

In contrast with the previous studies, Parmar et al. [34] performed a robust study using feature dimensionality reduction based on consensus clustering for a prognostic signature in 422 lung and 136 head/neck cancer patients, which were then externally validated in an additional 225 lung and 95 head/neck cases, respectively. For lung cancer staging and histology classification, they reported a final lung cancer radiomic signature with external AUCs of 0.64 and 0.64 , respectively. These AUCs are much lower than the previously cited works, but may be a more realistic estimation of the Radiomics model performance.

Some methodological deficiencies were also noted in new studies of histological classification. Patil et al. [22] demonstrated the extra value of textural radiomic features when classifying NSCLC histology, increasing the classification accuracy from $67 \%$ to 
$88 \%$ compared to shape and intensity features alone [22]. The results were based on balancing the dataset using selective resampling of the minority events (i.e. SMOTE ) with a 10-fold cross validation approach. Again, no external dataset was used to independently test accuracy. Separately, the results of $\mathrm{Wu}$ et al. [31] were consistent with Patil et al., in support of added value of radiomic features when classifying the histology of NSCLC, however Wu et al. also did not perform external validation of the algorithm.

Synthesis of Radiomics performance in predicting histology or cancer stage

Radiomic features appear to have potential for discrimination of malignant nodules and mediastinal lymph node involvement in NSCLC. The reported metrics initially look appealing (AUCs > o.83), but requires external validation to prove that such performance is generalizable. Radiomic features appear to have moderate utility for classification of NSCLC histology. One methodologically robust study by Parmar et al with large sample size and careful feature selection suggests an AUC of about 0.64 (externally validated) for staging and histology is the current limit of performance with hand-engineered Radiomics features. Two studies have been consistent in reporting that textural radiomic features could have added value beyond models that use only non-textural features.

\section{Radiomics for predicting treatment outcomes}

General characteristics of reviewed studies

Table 2 summarizes the general characteristics of the reviewed studies. The features extracted from a single modality were evenly split between CT [21,39, 46, 45,84,38,82,78,48] and PET [4 2,71, 43, 59, 71, 72, 44, 74, 75, 76, 77$]$. There was only one study [78] that used both ${ }_{3} \mathrm{D}$ and time-resolved $\left({ }_{4} \mathrm{D}\right) \mathrm{CT}$ features. A further four studies 
examined a potential combination of PET-CT features [49, 40, 79, 8o]. A single study [81] considered 4D hybrid PET-CT.

\begin{tabular}{|c|c|c|c|c|}
\hline $\begin{array}{l}\text { Study Ref. } \\
\text { (author:yea } \\
\text { r) }\end{array}$ & $\begin{array}{c}\text { Sample } \\
\text { size }\end{array}$ & Intervention & Overall stage & Imaging \\
\hline Aerts:2014 & 422 & $\begin{array}{l}\text { Curative chemo- } \\
\text { radiotherapy }\end{array}$ & I-IV & CT \\
\hline Ahn:2015 & 91 & $\begin{array}{l}\text { Definitive chemo- } \\
\text { radiotherapy }\end{array}$ & III & $\mathrm{CT}$ \\
\hline Coroller:2015 & 98 & $\begin{array}{l}\text { Unspecified } \\
\text { radiotherapy }\end{array}$ & II-III & CT \\
\hline Coroller:2016 & 127 & $\begin{array}{l}\text { Neoadjuvant chemo- } \\
\text { radiotherapy }\end{array}$ & II-III & CT \\
\hline Dong:2016 & 58 & $\begin{array}{l}\text { Concurrent chemo- } \\
\text { radiotherapy }\end{array}$ & III & CT \\
\hline $\begin{array}{l}\text { Ganeshan:20 } \\
12\end{array}$ & 54 & $\begin{array}{l}\text { Surgery or } \\
\text { chemotherapy }\end{array}$ & I-IV & CT \\
\hline Huang:2016 & 282 & Surgical resection & IA-IIB & CT \\
\hline Huynh:2016 & 113 & Stereotactic radiotherapy & I-II & $\mathrm{CT}$ \\
\hline $\begin{array}{l}\text { Mattonen:20 } \\
15\end{array}$ & 22 & Stereotactic radiotherapy & I & CT \\
\hline $\begin{array}{l}\text { Mattonen:20 } \\
16\end{array}$ & 45 & Stereotactic radiotherapy & I & CT \\
\hline Huynh:2017 & 112 & Stereotactic radiotherapy & I-IIA & $\begin{array}{c}\text { 3D \& 4D } \\
\text { CT }\end{array}$ \\
\hline $\begin{array}{l}\text { Carvalho:201 } \\
3\end{array}$ & 220 & Curative radiotherapy & I-IIIB & PET \\
\hline Cheng:2016 & 56 & $\begin{array}{l}\text { Several curative } \\
\text { treatments }\end{array}$ & I-IIIB & PET \\
\hline
\end{tabular}




\begin{tabular}{|c|c|c|c|c|}
\hline Cook:2013 & 53 & $\begin{array}{l}\text { Definitive chemo- } \\
\text { radiotherapy }\end{array}$ & I-IIIB & PET \\
\hline Cook:2015 & 47 & $\begin{array}{l}\text { Chemotherapy only } \\
\text { (erlonitib) }\end{array}$ & IIIB-IV & PET \\
\hline Fried:2014 & 91 & $\begin{array}{l}\text { Definitive photon } \mathrm{RT}+/- \\
\text { platinum }\end{array}$ & III & PET \\
\hline Fried:2016a & 225 & $\begin{array}{l}\text { Curative chemo- } \\
\text { radiotherapy }\end{array}$ & III & PET \\
\hline Fried:2016b & 195 & Definitive radiotherapy & III & PET \\
\hline $\begin{array}{l}\text { Lovinfosse:2 } \\
\text { o16 }\end{array}$ & 63 & Stereotactic radiotherapy & I & PET \\
\hline Ohri:2016 & 201 & $\begin{array}{l}\text { Concurrent chemo- } \\
\text { radiotherapy }\end{array}$ & III & PET \\
\hline Pyka:2015 & 45 & Stereotactic radiotherapy & $\mathrm{I}$ & PET \\
\hline Wu:2016 & 101 & Stereotactic radiotherapy & $\mathrm{I}$ & PET \\
\hline $\begin{array}{l}\text { Desseroit:201 } \\
6\end{array}$ & 116 & $\begin{array}{l}\text { Surgery +/- (chemo)- } \\
\text { radiotherapy }\end{array}$ & I-III & $\begin{array}{l}\text { PET \& } \\
\text { CT }\end{array}$ \\
\hline Hayano:2016 & 35 & $\begin{array}{l}\text { Anti-angiogenic } \\
\text { chemotherapy }\end{array}$ & IIIB-IV & $\begin{array}{l}\text { PET \& } \\
\text { CT }\end{array}$ \\
\hline Vaidya:2012 & 27 & $\begin{array}{l}\text { Radiotherapy incl. } \\
\text { stereotactic }\end{array}$ & I-IV & $\begin{array}{l}\text { PET \& } \\
\text { CT }\end{array}$ \\
\hline Win:2013 & 56 & $\begin{array}{l}\text { Palliative and radical } \\
\text { treatments }\end{array}$ & I-IV & $\begin{array}{l}\text { PET \& } \\
\text { CT }\end{array}$ \\
\hline Li:2015 & 25 & $\begin{array}{l}\text { Concurrent chemo- } \\
\text { radiotherapy }\end{array}$ & II-III & $\begin{array}{l}\text { 4D PET- } \\
\text { CT }\end{array}$ \\
\hline
\end{tabular}

Table 2. Case characteristics of included non-small cell lung cancer studies. Sample size refers to the model development cohort. Abbreviations for imaging modality: (CT) computed x-ray tomography, (PET) positron emission tomography, $(3 \mathrm{D})$ threedimensional and $(4 \mathrm{D})$ four-dimensional i.e. time-resolved. 
Sample sizes for the studies ranges from as few as 25, up to the largest study consisting of 422 individual subjects. This already indicates one of the major problems facing radiomic studies, where the number of features incorporated into optimal model selection typically exceeds the number of events by a factor of 10 or greater.

Disease stages (according to the American Joint Committee for Cancer - AJCC - scale) of NSCLC ranged from I up to IV. The vast majority of the stage II-IV studies involved patients treated with radiotherapy, with or without addition of chemotherapy. Stereotactic radiotherapy was used in seven studies for inoperable Stage I or Stage II. One study [49] used patients treated with combined surgery and chemo-radiotherapy. Only 3 studies $[38,40,82]$ involved a combination of surgery with or without chemotherapy. A single study [83] considered patients with advanced disease treated by erlonitib only.

Selection bias towards radiotherapy patients with either advanced or locally advanced lung cancer is most likely due to the dependence of radiomic analysis on pre-defined gross tumor volume annotations. These ROIs are most likely to have been manually created in clinical routine on CT images made for radiotherapy treatment planning purposes. The preponderance of radiotherapy cases also suggests that the patient population under review may, on average, have larger tumor volumes and may also be in poorer overall health than the general lung cancer population.

\section{Overall survival}

There were 12 original research articles that addressed overall survival (OS) as an outcome of interest $[39,84,43,44,72,73,75,40,21,49,85,41]$. The majority of these OS models did not report on performance in an independent external cohort except for Aerts et al. [21] that used multiple external cohorts from clinics different than the ones used to procure the training data. The studies by Fried et al. [44] and Ohri et al. [75] used a holdout validation set kept apart from the training data. Of the above, Aerts et al. [21] and Fried et al. [44] reported conformance indices; Ohri et al. [75] only confirmed 
statistical significance with a log-rank test. The conformance indices ranged from $0.65-$ o.69 for Aerts et al. to 0.62 for Fried et al. The remaining studies that did not test performance in an external or holdout validation set, reported hazard ratio (HR) ranging from 1 to 17 , with four of the studies [39, 84, 42, 43] reporting HRs less than 4. Desseroit et al. [49] estimated a HR of 4.9 and 17 for difference in overall survival in Stage II and Stage III disease, respectively, for a signature comprising one PET feature and one CT radiomic feature.

In the articles reviewed, the number of features per event of interest and the method of Radiomics feature selection was not uniformly well documented. Eight articles [39, 84, $43,44,72,73,75,40]$ did not provide the information to understand how many features were used for model tuning versus how many death (or alive, whichever was smaller) events were present in their cohort. Aerts et al. [21] did not specify the event rate either, but it was possible to infer this from the survival curves; there were 244 deaths and 440 Radiomics features, which were then reduced to a signature of four features through a well-documented process of feature selection through test-retest and inter observer agreement. However, the final choice of the top feature from each feature class was somewhat arbitrary, and the authors could not rule out association with gross tumour volume, which was subsequently shown to be potential confounder of the radiomic signature [85].

Carvalho et al. [42] placed 150 events and 47 features into an unsupervised clustering model, and returned a HR estimate of 1 ; there was no feature selection applied due to the unsupervised learning method. Desseroit et al. [49] had the most favorable ratio with 71 events for only 12 features, and then reported on a two-feature signature after applying test-retest repeatability, non-correlation and using a prior knowledge of useful features from another dataset.

The study by Ganeshan et al. [38] reported an odds ratio (OR) estimate of 56.4, in a dataset with 27 events and 8 Radiomics features, but the optimal feature set was selected 
as the features with the best AUC value for predicting the outcome and there was no external validation either in a holdout or independent fashion.

Ahn et al. [39] investigated the association of textural features with OS in 91 NSCLC patients undergoing $(n=91)$ definitive concomitant chemoradiotherapy. The study concluded that there was no significant difference in 3-year overall survival based on the tumor stage or histology and the mean 3-year OS was 24 months. However, this study has a potential selection bias by selecting only the subjects with good performance with concomitant chemoradiotherapy and the interobserver variability of tumor segmentation leading to different texture parameters was not evaluated.

Hyun Jung Yoon et al. [50] tried to establish a relation between Radiomics and gene expression in decoding the tumor phenotype. They showed that quantitative features help in discriminating fusion-positive tumors from fusion negative tumors in ALK/ROSI/RET without external data validation.

Another study by Hayano K et al. [40] evaluated different imaging bio markers including 18F-FDG PET, CT perfusion, and CT texture analysis to predict the survival of patients with advanced NSCLC treated with antiangiogenic chemotherapy. CT texture analysis was found to be the optimal imaging biomarker for predicting the survival of patients. In this study it is assumed that relative texture analysis allows the effect of variations in acquisition parameters on lung tumor texture to be minimized, therefore making this approach applicable across centers, however this was not validated.

A study by Balagurunathan et al. [41] took an approach to explore how stable the radiomic features are, by applying a test-retest concordance correlation coefficient metric and used the stable features to predict radiologist prognostic scores. In addition, the outcome of this study showed that one of the texture features, run-length gray-level nonuniformity, could separate the samples into survival groups with borderline statistical significance $(P \leq .046)$. A study by Fried et al $(n=195)$ evaluated if quantitative feature extraction from pretreatment positron emission tomography (PET) has the 
ability to enhance overall survival risk stratification compared to conventional prognostic factors in patients with stage III NSCLC [44]. It was concluded that the CI index improved marginally from 0.58 to 0.62 by considering the quantitative features. The disease solidity and primary tumor energy features from the co-occurrence matrix were found to be the strongest prognostic indicators.

\section{Disease and progression free survival}

We reviewed four original research publications relating to progression-free survival (PFS) $[84,43,44,40]$. Two on disease-free survival (DFS) $[82,74]$ and three that were related to disease-specific survival (DSS) [71, 74 and 76]. Of these, only Huang et al [82] set aside a hold-out set for validation; none of the abovementioned studies reported on validation in an independent external cohort. Huang et al estimated the conformance index for a Radiomics signature predicting DFS at $\mathbf{0 . 7 2}$ versus a clinical-only nomogram at o.69. But, given the lack of external validation, it is hard to measure the added value of the Radiomics signature. Lovinfosse et al estimated a similar HR for both DFS (o.83) and DSS (o.82) based on their own in-house Radiomics program in Python. In contrast, Cheng et al. [71] and Pyka et al. [76] reported much larger HR for DSS ranging from 2.5 to $7 \cdot 5$.

Upon inspection, both Cheng et al and Pyka et al. used around 15 image-derived metrics for a total of 47 and 12 events, in their respective cohorts. Huang started with a total of 136 explanatory variables including 4 clinical variables, for a total of 97 events. None of the other authors provided information about the number of events in their cohort, but did provide information about the number of features examined (see Table 3 and Table 4). Given the relatively low number of events in these studies, it is essential to consider the feature selection used. 


\begin{tabular}{|c|c|c|c|c|}
\hline $\begin{array}{l}\text { Study Ref. } \\
\text { (Author:Yea } \\
\text { r) }\end{array}$ & $\begin{array}{c}\text { Features Per } \\
\text { Patient }\end{array}$ & $\begin{array}{c}\text { Outcomes } \\
\text { (Event } \\
\text { Rates) }\end{array}$ & $\begin{array}{c}\text { Dimensionality } \\
\text { Reduction }\end{array}$ & $\begin{array}{c}\text { Type Of } \\
\text { Validatio } \\
\text { n }\end{array}$ \\
\hline Aerts:2014 & 440 features & $\begin{array}{l}\text { OS (244 } \\
\text { events) }\end{array}$ & $\begin{array}{l}\text { Stability ranks in } \\
\text { independent image } \\
\text { series }\end{array}$ & $\begin{array}{c}\text { Lung } \\
(\mathrm{n}=314) \\
\mathrm{H} \& \mathrm{~N} \\
(\mathrm{n}=184)\end{array}$ \\
\hline Ahn:2015 & Not specified & $\begin{array}{l}\text { OS (48 } \\
\text { events) }\end{array}$ & $\begin{array}{l}\text { ROC utility, } \\
\text { backwards Cox } \\
\text { regression }\end{array}$ & None \\
\hline Coroller:2015 & $\begin{array}{l}635 \text { Radiomics } \\
\text { incl } 6 \text { clinical } \\
\text { factors }\end{array}$ & $\begin{array}{l}\text { DM (64 } \\
\text { events), } \\
\text { OS (not } \\
\text { stated) }\end{array}$ & $\begin{array}{l}\text { Relevance and non- } \\
\text { redundancy }\end{array}$ & $\begin{array}{l}\text { Lung } \\
(\mathrm{n}=84)\end{array}$ \\
\hline Coroller:2016 & $\begin{array}{c}18 \text { incl } 3 \text { simple } \\
\text { features }\end{array}$ & $\begin{array}{l}\text { pCR (27 } \\
\text { events), } \\
\text { MRD (33 } \\
\text { events), } \\
\text { GRD (67 } \\
\text { events) }\end{array}$ & $\begin{array}{c}\text { Test-retest and } \\
\text { principal component } \\
\text { analysis }\end{array}$ & $\begin{array}{c}\text { Cross- } \\
\text { validation }\end{array}$ \\
\hline Dong:2016 & $\begin{array}{l}10 \text { incl } 2 \text { SUV } \\
\text { measures }\end{array}$ & $\begin{array}{l}\text { CR, PFS and } \\
\text { OS (not } \\
\text { stated) }\end{array}$ & $\begin{array}{c}\text { Features selected in } \\
\text { prior study }\end{array}$ & None \\
\hline
\end{tabular}




\begin{tabular}{|c|c|c|c|c|}
\hline $\begin{array}{l}\text { Ganeshan:201 } \\
2\end{array}$ & $\begin{array}{l}\text { *8 incl } \\
\text { SUVmax and } \\
\text { clinical stage }\end{array}$ & $\begin{array}{l}\text { OS (27 } \\
\text { events) }\end{array}$ & Selected by AUC & None \\
\hline Huang:2016 & $\begin{array}{c}136 \text { incl } 4 \\
\text { clinical factors }\end{array}$ & $\begin{array}{l}\text { DFS (97 } \\
\text { events) }\end{array}$ & $\begin{array}{c}\text { Penalized Cox multi- } \\
\text { regression }\end{array}$ & $\begin{array}{c}\text { Hold-out } \\
\text { set }\end{array}$ \\
\hline Huynh:2016 & $\begin{array}{l}1605 \text { incl } 4 \\
\text { clinical factors }\end{array}$ & $\begin{array}{l}\text { OS (59 } \\
\text { events), } \\
\text { DM ( } 23 \\
\text { events), } \\
\text { LRR (24 } \\
\text { events) }\end{array}$ & $\begin{array}{c}\text { Test-retest and } \\
\text { principal component } \\
\text { analysis }\end{array}$ & $\begin{array}{l}\text { Cross- } \\
\text { validation }\end{array}$ \\
\hline $\begin{array}{l}\text { Mattonen:201 } \\
5\end{array}$ & 5 features & $\begin{array}{l}\text { LR (24 } \\
\text { events) }\end{array}$ & $\begin{array}{l}\text { No selection } \\
\text { performed }\end{array}$ & $\begin{array}{l}\text { Cross- } \\
\text { validation }\end{array}$ \\
\hline $\begin{array}{l}\text { Mattonen:201 } \\
6\end{array}$ & 104 excl shape & $\begin{array}{c}\text { LR at } 6 \\
\text { months (15 } \\
\text { events) }\end{array}$ & $\begin{array}{l}\text { Forward stepwise } \\
\text { logistic regression }\end{array}$ & $\begin{array}{c}\text { Cross- } \\
\text { validation }\end{array}$ \\
\hline Huynh:2017 & $\begin{array}{c}646 \text { incl } 2 \\
\text { simple metrics }\end{array}$ & $\begin{array}{l}\text { OS (59 } \\
\text { events), } \\
\text { DM ( } 23 \\
\text { events), } \\
\text { LRR (24 } \\
\text { events) }\end{array}$ & $\begin{array}{c}\text { Test-retest and } \\
\text { principal component } \\
\text { analysis }\end{array}$ & $\begin{array}{c}\text { Cross- } \\
\text { validation }\end{array}$ \\
\hline Carvalho:2013 & 47 features & OS(150) & $\begin{array}{c}\text { Unsupervised } \\
\text { clustering }\end{array}$ & None \\
\hline
\end{tabular}

42 | Prognostic and Prediction Modelling with Radiomics for Non-Small Cell Lung Cancer 


\begin{tabular}{|c|c|c|c|c|}
\hline Cheng:2016 & $\begin{array}{l}15 \text { incl SUV } \\
\text { measures }\end{array}$ & $\begin{array}{l}\text { DSS (47 } \\
\text { events) }\end{array}$ & $\begin{array}{c}\text { ROC utility, stepwise } \\
\text { Cox regression }\end{array}$ & None \\
\hline Cook:2013 & $\begin{array}{l}13 \text { incl SUV } \\
\text { and clinical } \\
\text { factors }\end{array}$ & $\begin{array}{c}\text { RECIST, OS, } \\
\text { PFS and LPFS } \\
\text { (not stated) }\end{array}$ & $\begin{array}{l}\text { ROC utility, stepwise } \\
\text { Cox regression }\end{array}$ & None \\
\hline Cook:2015 & 14 incl SUV & OS & $\begin{array}{l}\text { backward } \\
\text { selection }\end{array}$ & None \\
\hline Fried:2014 & $\begin{array}{l}198 \text { Radiomics } \\
\text { incl } 13 \text { clinical } \\
\text { factors }\end{array}$ & $\begin{array}{l}\text { OS, LRF and } \\
\text { DM (not } \\
\text { stated) }\end{array}$ & $\begin{array}{l}\text { Test-retest, penalized } \\
\text { Cox regression }\end{array}$ & $\begin{array}{c}\text { Cross- } \\
\text { validation }\end{array}$ \\
\hline Fried:2016a & 2 features & $\begin{array}{l}\text { OS, PFS (not } \\
\text { stated) }\end{array}$ & $\begin{array}{c}\text { Features selected in } \\
\text { prior study }\end{array}$ & None \\
\hline Fried:2016b & $\begin{array}{l}38 \text { incl } 11 \\
\text { clinical factors }\end{array}$ & $\begin{array}{l}\text { OS (not } \\
\text { stated) }\end{array}$ & $\begin{array}{l}\text { Penalized Cox multi- } \\
\text { regression }\end{array}$ & $\begin{array}{c}\text { Cross- } \\
\text { validation }\end{array}$ \\
\hline $\begin{array}{l}\text { Lovinfosse:20 } \\
16\end{array}$ & $\begin{array}{l}20 \text { incl SUV } \\
\text { measures }\end{array}$ & $\begin{array}{l}\text { OS (27 } \\
\text { events), } \\
\text { DSS (12 } \\
\text { events), } \\
\text { DFS (25 } \\
\text { events) }\end{array}$ & Univariate selection & None \\
\hline Ohri:2016 & $\begin{array}{c}45 \text { incl } 2 \text { SUV } \\
\text { measures }\end{array}$ & $\begin{array}{l}\text { OS (not } \\
\text { stated) }\end{array}$ & $\begin{array}{l}\text { Penalized Cox } \\
\text { regression }\end{array}$ & $\begin{array}{l}\text { Cross- } \\
\text { validation }\end{array}$ \\
\hline Pyka:2015 & $\begin{array}{c}10 \text { incl } 4 \text { SUV, } 1 \\
\text { CT simple } \\
\text { metrics }\end{array}$ & $\begin{array}{l}\text { LR, NM, DM } \\
\text { (not } \\
\text { specified), }\end{array}$ & Not specified & None \\
\hline
\end{tabular}




\begin{tabular}{|c|c|c|c|c|}
\hline & & $\begin{array}{l}\text { DSS (12 } \\
\text { events), } \\
\text { OS (28 } \\
\text { events) }\end{array}$ & & \\
\hline Wu:2016 & 70 features & $\begin{array}{l}\text { DM (11 } \\
\text { events), } \\
\text { OS (32 } \\
\text { deaths) }\end{array}$ & $\begin{array}{l}\text { Reproducibility, non- } \\
\text { correlation, penalized } \\
\text { Cox }\end{array}$ & $\begin{array}{l}\text { Lung } \\
(\mathrm{n}=31)\end{array}$ \\
\hline $\begin{array}{l}\text { Desseroit:201 } \\
6\end{array}$ & $\begin{array}{l}12 \text { incl SUV } \\
\text { measures and } \\
\text { clinical stage }\end{array}$ & $\begin{array}{l}\text { OS (71 } \\
\text { events) }\end{array}$ & $\begin{array}{l}\text { Test-retest, previous } \\
\text { study and non- } \\
\text { correlation }\end{array}$ & None \\
\hline Hayano:2016 & $\begin{array}{l}7 \text { total (only } 2 \\
\text { were } \\
\text { Radiomics) }\end{array}$ & $\begin{array}{l}\text { OS, PFS (not } \\
\text { stated) }\end{array}$ & $\begin{array}{l}\text { Prior knowledge and } \\
\text { Cox regression }\end{array}$ & None \\
\hline Vaidya:2012 & $\begin{array}{l}17 \text { incl SUV } \\
\text { and CT } \\
\text { measures }\end{array}$ & $\begin{array}{l}\text { LRR and LF } \\
\text { (not stated) }\end{array}$ & Not specified & $\begin{array}{l}\text { Cross- } \\
\text { validation }\end{array}$ \\
\hline Win:2013 & $\begin{array}{l}{ }^{*} 6 \text { textural incl } \\
\text { CT perfusion }\end{array}$ & $\begin{array}{l}\text { OS (27 } \\
\text { events) }\end{array}$ & Not specified & $\begin{array}{l}\text { Lung } \\
(n=66)\end{array}$ \\
\hline Li:2015 & $\begin{array}{l}37 \text { in total for } \\
\text { nodes and } \\
\text { tumour }\end{array}$ & $\begin{array}{c}2 \text { yr LR (5 } \\
\text { events), } \\
\text { overall } \\
\text { recurrence } \\
\text { (15 events) }\end{array}$ & $\begin{array}{l}\text { Non-correlation and } \\
\text { stepwise (backwards) } \\
\text { elimination }\end{array}$ & $\begin{array}{c}\text { Cross- } \\
\text { validation }\end{array}$ \\
\hline
\end{tabular}

Table 3. Statistical analytic aspects of the included non-small cell lung cancer studies. The asterisk $\left(^{*}\right)$ indicated radiomic features extracted from a single axial slice only (2D analysis). Abbreviations for clinical outcomes : (OS) overall survival, (DSS) disease-specific survival, (PFS) progression-free survival, (LPFS) local progression free survival, (DM) distant metastasis, (pCR) pathological complete response, (MRD) microscopic residual disease, (GRD) 
gross residual disease, (CR) complete response, (LRF) locoregional failure, (LRR) locoregional recurrence, (LR) local recurrence, (DFS) disease free survival and (NM) nodal metastasis.

(AUC) Area Under Curve, (HR) Hazard Ratio, (MCC) Matthews correlation coefficient

Huang used a penalized Cox regression approach, but it is unclear how many features were initially allowed for tuning against a Cox regression. Without additional details about multiple bootstrap resampling within the cohort, there may be a high risk that the selected features themselves become too specific to the training cohort and holdout validation only very slightly mitigates this risk. Both Dong [84] and Fried [44] used features that had been tested in a previous study. The other studies variously used either ROC utility [71] or prior knowledge [40] to select features for stepwise Cox regression, or used stepwise regression outright [43]. Lovinfosse et al. [74] used only univariate Cox regression to select features, which has a high risk of an over-parameterized multivariate model, and Pyka et al. [76] did not report any feature selection approach.

\begin{tabular}{|c|c|c|c|}
\hline $\begin{array}{l}\text { Study Ref. } \\
\text { (Author:Year) }\end{array}$ & $\begin{array}{c}\text { Features } \\
\text { Extraction }\end{array}$ & Predictive Features & $\begin{array}{c}\text { Model } \\
\text { Performance }\end{array}$ \\
\hline Aerts:2014 & $\begin{array}{l}\text { In-house } \\
\text { MATLAB }\end{array}$ & $\begin{array}{c}\text { Energy, compactness, gray level } \\
\text { non-uniformity, wavelet-HLH } \\
\text { gray level non-uniformity }\end{array}$ & $\begin{array}{l}\text { OS }\left(\mathrm{CI}=0.69 \mathrm{H} \& \mathrm{~N}_{1}\right) \\
\text { OS }(\mathrm{CI}=0.69 \mathrm{H \& N} 2) \\
\text { OS }(\mathrm{CI}=0.65 \text { Lung2 })\end{array}$ \\
\hline Ahn:2015 & $\begin{array}{c}\text { In-house } \\
\mathrm{C}++\end{array}$ & Entropy, skewness and mean & $\begin{array}{c}\text { OS (Entropy, HR:2.31) } \\
\text { OS (Skewness, } \\
\text { HR:1.92) } \\
\text { OS (Mean, adj } \\
\text { HR:1.93) }\end{array}$ \\
\hline Coroller:2015 & $\begin{array}{l}\text { In-house } \\
\text { MATLAB }\end{array}$ & $\begin{array}{l}\text { Wavelet-HHL skewness, cluster } \\
\text { shade, LoG5mm2D skewness, } \\
\text { clinical stage and grade }\end{array}$ & $\mathrm{DM}(\mathrm{CI}=0.61)$ \\
\hline
\end{tabular}




\begin{tabular}{|c|c|c|c|}
\hline Coroller:2016 & $\begin{array}{l}\text { In-house } \\
\text { MATLAB }\end{array}$ & $\begin{array}{c}\text { Signature consisting of } 7 \text { features } \\
\text { and } 3 \text { image metrics }\end{array}$ & $\begin{array}{c}\text { pCR }(\mathrm{AUC}=0.63), \\
\text { GRD }(\mathrm{AUC}> \\
\text { o.6), Tumors that } \\
\text { didn't respond }( \\
\text { Entropy) }(\mathrm{AUC}=0.61)\end{array}$ \\
\hline Dong:2016 & $\begin{array}{l}\text { In-house } \\
\text { MATLAB }\end{array}$ & Percent change in contrast & $\begin{array}{c}\text { PFS }(\mathrm{HR}=0.476) \\
\text { OS }(\mathrm{HR}=0.519)\end{array}$ \\
\hline $\begin{array}{l}\text { Ganeshan:201 } \\
2\end{array}$ & $\begin{array}{l}\text { In-house } \\
\text { TexRAD }\end{array}$ & $\begin{array}{c}\text { Coarse LoG uniformity and PET } \\
\text { stage }\end{array}$ & $\begin{array}{c}\text { OS (Radiomics OR = } \\
56.4 \\
\text { vs PET-Stage OR = } \\
3.85)\end{array}$ \\
\hline Huang:2016 & $\begin{array}{l}\text { In-house } \\
\text { MATLAB }\end{array}$ & $\begin{array}{c}\text { Kurtosis, uniformity and } \\
\text { homogeneity }\end{array}$ & $\begin{array}{c}\text { DFS (CI = 0.72 vs } \\
\text { clinical nomogram CI } \\
=0.69)\end{array}$ \\
\hline Huynh:2016 & $\begin{array}{l}\text { In-house } \\
\text { MATLAB }\end{array}$ & $\begin{array}{c}\text { (DM) LLH range and LHL total } \\
\text { energy }\end{array}$ & $\mathrm{DM}(\mathrm{CI}=0.67)$ \\
\hline $\begin{array}{l}\text { Mattonen:201 } \\
5\end{array}$ & $\begin{array}{l}\text { In-house } \\
\text { MATLAB }\end{array}$ & $\begin{array}{c}\text { First order features ; four } \\
\text { second-order texture features } \\
\text { based on a GLCM: energy, } \\
\text { entropy, inertia, and correlation }\end{array}$ & $\begin{array}{l}\text { Recurrences (Semi- } \\
\text { automatic } \\
\text { segmentation AUC } \\
\text { o.7-0.73 vs Manual } \\
\text { Segmentaion AUC } \\
\text { :0.64 ) }\end{array}$ \\
\hline $\begin{array}{l}\text { Mattonen:201 } \\
6\end{array}$ & $\begin{array}{l}\text { In-house } \\
\text { MATLAB }\end{array}$ & $\begin{array}{l}4 \text { GLCM features, minimum, } \\
\text { gray level non-uniformity }\end{array}$ & $\mathrm{LR}(\mathrm{AUC}=0.85)$ \\
\hline
\end{tabular}




\begin{tabular}{|c|c|c|c|}
\hline Huynh:2017 & $\begin{array}{l}\text { In-house } \\
\text { MATLAB }\end{array}$ & $\begin{array}{c}\text { (DM) Signature from free- } \\
\text { breathing and intensity- } \\
\text { projection }\end{array}$ & $\begin{array}{l}\text { DM (median CI = } \\
\text { o.667, intensity } \\
\text { projection image) }\end{array}$ \\
\hline Carvalho:2013 & $\begin{array}{l}\text { In-house } \\
\text { MATLAB }\end{array}$ & $\begin{array}{l}\text { Relative volume above higher } \\
\qquad \text { SUV (80 \%) }\end{array}$ & OS (HR 1.02)? \\
\hline Cheng:2016 & $\begin{array}{l}\text { CGITA in } \\
\text { MATLAB }\end{array}$ & $\begin{array}{l}\text { Entropy, local entropy and } \\
\text { coarseness }\end{array}$ & $\begin{array}{c}\text { DSS (Entropy, HR = } \\
2.47) \\
\text { DSS (Local entropy, } \\
\text { HR = 3.4) DSS } \\
(\text { Coarseness, HR = } \\
0.28)\end{array}$ \\
\hline Cook:2013 & $\begin{array}{c}\text { Not } \\
\text { specified }\end{array}$ & Coarseness & $\begin{array}{c}\mathrm{pCR}(\mathrm{AUC}=0.8 \mathrm{o}, \mathrm{HR} \\
=0.91)\end{array}$ \\
\hline Cook:2015 & $\begin{array}{l}\text { In-house } \\
\text { MATLAB }\end{array}$ & $\begin{array}{l}\text { first-order entropy, high-order } \\
\text { contrast at } 6 \text { weeks }\end{array}$ & $\begin{array}{l}\text { OS(Entropy, } \mathrm{HR}=1.36 \\
\text { Contrast, } \mathrm{HR}=1.08 \text { ) }\end{array}$ \\
\hline Fried:2014 & $\begin{array}{l}\text { In-house } \\
\text { MATLAB }\end{array}$ & $\begin{array}{l}\text { Multiple different signatures for } \\
\text { each outcome }\end{array}$ & $\begin{array}{c}\mathrm{OS}(\mathrm{p}=0.046) \text {, local- } \\
\text { regional control (LRC), } \\
\text { and }(\mathrm{p}=\mathrm{o} .01) \text {, freedom } \\
\text { from distant } \\
\text { metastases }(\mathrm{FFDM}) \\
\quad(\mathrm{p}=0.005)\end{array}$ \\
\hline Fried:2016a & $\begin{array}{l}\text { IBEX in } \\
\text { MATLAB }\end{array}$ & $\begin{array}{l}\text { Co-occurrence matrix energy } \\
\text { and solidity }\end{array}$ & $\begin{array}{l}\text { OS ( } 74 \text { Gy vs } 60 \text { to } 70 \\
\text { Gy Log-rank p<0.02.) }\end{array}$ \\
\hline Fried:2016b & $\begin{array}{l}\text { IBEX in } \\
\text { MATLAB }\end{array}$ & $\begin{array}{l}\text { Co-occurrence matrix energy } \\
\text { and solidity }\end{array}$ & $\begin{array}{l}\mathrm{OS}(\mathrm{Cl}=0.62 \text { vs clinical } \\
\text { factors } \mathrm{Cl}=0.58)\end{array}$ \\
\hline
\end{tabular}




\begin{tabular}{|c|c|c|c|}
\hline $\begin{array}{l}\text { Lovinfosse:201 } \\
6\end{array}$ & $\begin{array}{l}\text { In-house } \\
\text { PYTHON }\end{array}$ & $\begin{array}{c}\text { Dissimilarity (DSS and DFS), } \\
\text { none associated with OS }\end{array}$ & $\begin{aligned} \text { DSS }(\mathrm{HR} & =0.822) \mathrm{DFS} \\
(\mathrm{HR}, & =0.834)\end{aligned}$ \\
\hline Ohri:2016 & $\begin{array}{l}\text { In-house } \\
\text { MATLAB }\end{array}$ & Sum mean and MTV & $\begin{array}{c}\text { OS (Log-rank p }< \\
\text { o.0o1) }\end{array}$ \\
\hline Pyka:2015 & $\begin{array}{c}\text { Not } \\
\text { specified }\end{array}$ & (DSS) Local entropy & $\begin{array}{c}\mathrm{LR}(\mathrm{AUC}=0.872(\mathrm{HR}= \\
7.48)\end{array}$ \\
\hline Wu:2016 & $\begin{array}{l}\text { In-house } \\
\text { MATLAB }\end{array}$ & $\begin{array}{l}\text { Filtered cluster shade and } \\
\qquad \text { SUVmax }(2 \mathrm{~mL})\end{array}$ & $\begin{array}{c}\mathrm{DM}(\mathrm{HR}=6.9, \mathrm{CI}= \\
0.8 \mathrm{o})\end{array}$ \\
\hline $\begin{array}{l}\text { Desseroit:201 } \\
6\end{array}$ & $\begin{array}{c}\text { Not } \\
\text { specified }\end{array}$ & $\begin{array}{c}\text { PET local entropy and CT zone } \\
\text { percentage }\end{array}$ & $\begin{array}{c}\text { OS, Stage II }(\mathrm{HR}=4.9) \\
\text { OS, Stage III }(\mathrm{HR}= \\
17.4)\end{array}$ \\
\hline Hayano:2016 & $\begin{array}{l}\text { TexRAD } \\
\text { (licensed) }\end{array}$ & $\begin{array}{l}\text { (PFS) Mean of positive pixels } \\
\text { and entropy, (OS) SUVmax }\end{array}$ & $\begin{array}{c}\text { PFS (Log-rank, p = } \\
\text { o.01) } \\
\text { OS (Log-rank, } \\
\text { p<0.04) }\end{array}$ \\
\hline Vaidya:2012 & $\begin{array}{c}\text { Not } \\
\text { specified }\end{array}$ & PET V8o\% and CT V70\% & $\begin{array}{l}\mathrm{LRR}(\mathrm{rs}=0.4854) \\
\operatorname{LF}(\mathrm{rs}=0.5908)\end{array}$ \\
\hline Win:2013 & $\begin{array}{c}\text { Not } \\
\text { specified }\end{array}$ & $\begin{array}{l}\text { Heterogeneity, clinical stage and } \\
\text { permeability }\end{array}$ & $\begin{array}{l}\text { (Heterogeneity: HR, } \\
\text { 412.241; 95\% CI; 1.45- } \\
\text { 103.05; } \\
\text { P=0.021; Clinical stage: } \\
\text { HR, 5.02;95\%CI: 1.89- } \\
\text { 13.32; P= }\end{array}$ \\
\hline
\end{tabular}




\begin{tabular}{|c|c|c|c|}
\hline & & & $\begin{array}{l}\text { o.001; Permeability: } \\
\text { HR, 6.01; 95\% CI; 2.34- } \\
15.41 ; \text { P <0.001) }\end{array}$ \\
\hline \multirow{2}{*}{ Li:2015 } & $\begin{array}{c}\text { In-house } \\
\text { MATLAB }\end{array}$ & $\begin{array}{r}\text { (LR) Signature of 8 features incl } \\
\text { SUVmax and volume, (OS) SUV } \\
\text { measure of node volume }\end{array}$ & $\begin{array}{c}\text { LR (MCC = 0.07) } \\
\text { Recurrence (MCC } \\
\text { o.29) }\end{array}$ \\
\hline
\end{tabular}

Table 4. Model performance.

List of abbreviations: (OS) Overall survival, (OR) Odds ratio, (pCR) Pathologic complete response, (PFS) Progression-free survival, LR (Local Recurrence), (DSS) Disease-specific survival 
A study by Carvalho et al. [42] measured the use of radiomic features for the early assessment of therapeutic response in NSCLC. They considered radiomic analysis on metabolic uptake patterns of repeated FDG-PET to assess the early response. The outcome of model suggested that early response metrics based on changes in metabolism measured with FDG-PET by radiomic analysis, had prognostic value with borderline significance $(\mathrm{P}=0.05)$ even before anatomic changes become noticeable, however there was no evidence that it is true for all the different tumor stages.

A 53 subjects based NSCLC study conducted by Cook et al. [43] showed a correlation between the radiomic features, response and survival after chemoradiotherapy. For disease-specific survival, texture Radiomics features showed a strong correlation with $\mathrm{P}$ $<0.007$. This study does not discuss the effect of the variation in scanner parameters on the texture features and variation of tumor segmentation on the output, which are important factors to consider, for ascertaining model efficacy across centers. Another similar study by Coroller et al. [45] investigated the role of Radiomics data to predict pathological response after neoadjuvant chemo radiation in patients with locally advanced NSCLC. It was observed that Radiomics performed better than conventional imaging features $(\mathrm{P}<0.05)$. But the AUC range lies around 0.6 , making this approach more like a base model rather than a prediction tool for pathological response.

Coroller et al. [46] conducted a study with 98 subjects to evaluate ComputedTomography (CT) radiomic features for their capability to predict distant metastasis (DM) for lung adenocarcinoma patients. It was observed that tumor volume was only moderately prognostic for DM, however their radiomic-signature had strong prognostic power in predicting DM which resulted in a significant improvement in the model output $\left(\mathrm{P}=1.56 \times 10^{\wedge}-11\right)$ with addition of radiomic features. Miles et al. [47] showed that patients with NSCLC frequently demonstrate differing clinical courses, even when they express the same tumor stage. They showed that a quantitative approach of generating information about tumor heterogeneity as reflected by the distribution of pixel values 
within the tumor could provide prognostic information for patients with NSCLC. However, both of the studies did not account for scanner variability in their approach.

The study by Mattonen SA et al. [48] in 2016 with 45 subjects, tried to assess the ability of physicians versus Radiomics to detect local recurrence after stereotactic ablative radiation therapy in early stage lung cancer. It was observed that Radiomics outperformed the physicians to detect the recurrence in terms of both sensitivity as well as specificity. Radiomics recorded an area under the receiver operating curve to be 0.85 . A study of Desseroit MC et al. ( $\mathrm{n}=116)$ developed a nomogram by exploiting intra tumor heterogeneity on $\mathrm{CT}$ and PET images acquisitions to identify patients with the poorest prognosis in stage I-III of NSCLC [49]. The patients with stage II and III showed improved nomogram-based stratification by considering the heterogeneity feature, which allowed identification of subjects with the poorest prognosis. This study clearly mentions the acquisition parameters and the protocol followed during the image acquisition, however it does not mention the reproducibility of the CT features. The study by Leijenaar RT et al. [51] studied the stability of the radiomic features by performing a test-retest study on 11 subjects before any treatment was provided. The results suggested that majority of the assessed features had high test-retest reproducibility and inter-observer agreement, which supports the hypothesis that radiomic features can be used as imaging biomarkers for treatment monitoring and outcome prediction. Parmar $\mathrm{C}$ et al. [52] $(\mathrm{n}=310)$ explored different machine learning concepts for their effect on the prognostics performance in Radiomics. They evaluated fourteen feature selection and twelve classification methods and concluded that choice of classification method is the most dominant source of performance variation. In this study a random forest algorithm along with a Wilcoxon test based feature selection method provided the best overall prognostic performance. This study used external datasets and a robust evaluation approach to arrive at the best choice for the feature selection and the classification algorithms. 


\section{Synthesis of Radiomics performance in treatment outcome prediction}

It is observed that in most of the studies, the number of events relative to features is low and there is not sufficient attention being paid to feature selection to ensure that "optimum features" are themselves not overly dependent on the particular cohort. There was no cohesive feature signature that establishes the best feature vector to determine treatment outcome. However, it seems that combinations of first order and texture features have better predictive power than first order features alone. The study by Mattonen SA et al. [48] highlights the Radiomics approach outperforming the physician to detect the recurrence, which paves a path to build diagnostic quality capable models. Moreover, only four studies have used the external validation approach to verify the efficacy of the models to predict the treatment outcome. Also, there are a few studies that provide a contrary view that tumor volume is only moderately prognostic and these claims need to be verified on large and multiple site data sets.

\section{Discussion of methodological issues encountered in reviewed studies}

Most of the models that are built using Radiomics features for predicting the treatment outcome or for clinical staging use a cross validation approach. Although, the approach of cross validation might seem valid and correct from a machine learning perspective, without external validation with data from other institutes there is a high risk of models being over fit. It is a better approach to perform cross validation on local data to tune the model parameters and finally validate the model with external data to the test the model robustness before reporting the results. It is also observed that there is no uniform approach for robust feature selection to identify the most significant features. It will also be useful to understand the contribution to the final signature by each of features, which is possible, if their weights in terms of predictive power are better reported in the feature selection approach. It is also quite possible that different feature selection approaches will highlight different features as most significant; hence, it is imperative that ensemble approaches are adapted among the feature selection methods to arrive at the subset of features that are most significant. Further, events in some studies are not clearly defined or not stated, making it difficult to trust the data points on which the models are built. It 
is good practice to be precise and transparent about the endpoints chosen and the event rate.

\section{Limitations of this review}

In this review, we have considered very specifically NSCLC as the disease under consideration and articles that are published in English language journals. In addition, we have used our discretion not to consider the articles where the number of subjects on which the results are reported were below 10.

\section{Conclusions}

Radiomics is currently used to predict overall survival, other tumor outcomes such as distant control and for identifying the histology and classification of the nodules found. It has shown a promising trend and potential in aiding both diagnosis and prognosis of NSCLC with the main challenges being reproducibility. It is observed that for NSCLC, the preferred modalities for Radiomics are CT and PET. Most of the studies have considered subjects belonging to all the four stages of NSCLC and the intervention planned is usually chemoradiotherapy with far fewer surgical resections. The number of features considered for predicting the endpoint varies from 7 to 1608 . Most of the studies have used a dimensionality reduction approach to arrive at the best subset of the features, with a test-retest approach being commonly used. Also, other algorithms such as Spearman rank, forward stepwise and Regularization/penalty are being used to identify the best features, from which it can be inferred that there is no popular choice or a strong motivation to select one dimensionality reduction technique over the other. It was also observed that the significant predictive features chosen are first order features (Energy, compactness, gray level non uniformity etc) and wavelets. The extractions of these features are carried out by custom implementations like MATLAB in most of the cases. Fewer studies have used commercial available tools such as TexRad and Medcalc and only one study has used an open source tool called IBEX. To perform segmentation of the region of interest, slicer $3 \mathrm{D}$ is being widely used across different studies if such annotation were not available from clinical routine. To arrive at the 
statistical metrics R and SPSS software are used. The metrics used to present the results vary based on the endpoint. For survival analysis c-index, Log Rank and hazard ratios are used and for the histology AUC is being reported. Most of the models built in the study are not validated on an external dataset, only four studies have performed external validation while others have used $\mathrm{k}$ - fold cross validation or a hold out approach. However, most of the studies have not mentioned explicitly how they have validated the model. The vast majority of studies did not share their training or test data, although utilities such as TCIA archive (https://www.cancerimagingarchive.net/) and BMIA (https://xnat.bmia.nl/) exist for such sharing and do have well annotated image datasets from large studies including in lung cancer.

In addition, investigations of feature repeatability and reproducibility are currently limited to a small number of cancer types. Reporting quality could be improved in regards to details of: feature extraction software, digital image manipulation (preprocessing) and cut-off value used to distinguish stable features. We recommend that authors publish image collections (including delineations) and feature extraction software as open access resources, to accelerate progress in clinical generalizability and independent validation of models. The positive aspects of all the studies points to a possible role of Radiomics features to improve the treatment outcome prediction in NSCLC, but a publication bias of only positive results likely exists. In due course, the research should focus on developing better explainable and robust models by combining multiple data sources to arrive at precision diagnosis in NSCLC leading to better health care outcomes.

Conflicts of Interest: Each author declares no potential conflict of interest. Grant funders have had no involvement in the preparation of this manuscript. 


\section{References}

[1] Malvezzi M, Bertuccio P, Rosso T, Rota M, Levi F, La Vecchia C, Negri E. "European cancer mortality predictions for the year 2015: does lung cancer have the highest death rate in EU women?", Ann Oncol. 2015 Apr;26(4):779-86. doi: 10.1093/annonc/mdvoo1. Epub 2015 Jan 26.

[2] A report on Lung Cancer (Non-Small Cell), American Cancer Society, 2016 (http://www.cancer.org/acs/groups/cid/documents/webcontent/oo3115-pdf.pdf).

[3] Hensing T, Chawla A, Batra R, Salgia R.”A personalized treatment for lung cancer: molecular pathways, targeted therapies, and genomic characterization". Adv Exp Med Biol. 2014;799:85-117. doi: 10.1007/978-1-4614-8778-4_5.

[4] Mok TS , "Personalized medicine in lung cancer: what we need to know".Nat Rev Clin Oncol. 2011 Aug 23;8(11):661-8. doi: 10.1038/nrclinonc.2011.126.

[5] 4.a Phlippe Lambin et.al Predicting outcomes in radiation oncology-multifactorial decision support systems," Nature Reviews Clinical Oncology volume 10, pages 27-40 (2013)"

[6] 4.b Emanuele Neri et.al Radiomics and liquid biopsy in oncology: the holons of systems medicine," Insights Imaging 2018

[7] Tomiyama N1, Yasuhara Y, Nakajima Y, Adachi S, Arai Y, Kusumoto M, Eguchi K, Kuriyama K, Sakai F, Noguchi M, Murata K, Murayama S, Mochizuki T, Mori K, Yamada, "CT-guided needle biopsy of lung lesions: a survey of severe complication based on 9783 biopsies in Japan”. K. Eur J Radiol. 2006 Jul;59(1):60-4. Epub 2006 Mar 10.

[8] Wu CC, Maher MM, Shepard JA. "Complications of CT-guided percutaneous needle biopsy of the chest: prevention and management”. AJR Am J Roentgenol. 2011 Jun;196(6):W678-82. doi: 10.2214/AJR.10.4659.

[9] Kristen M. CarrEmail author, Kevin Rosenblatt, Emanuel F. Petricoin and Lance A. Liotta ,"Genomic and proteomic approaches for studying human cancer: Prospects for true patient-tailored therapy", Human Genomics 20041:134 DOI: 10.1186/1479-7364-1-2134

[10] Institute of Medicine I. Evolution of Translational Omics. Lessons learned and the path forward. Washington, DC: National Academies Press, 2012.

[11] Clarke LP, Sriram RD, Schilling LB. "Imaging as a Biomarker: Standards for Change Measurements in Therapy workshop summary". Acad Radiol 2008;15(4):501-530.

[12] Shankar LK, Hoffman JM, Bacharach S, et al. "Consensus recommendations for the use of 18 F-FDG PET as an indicator of therapeutic response in patients in National Cancer Institute Trials". J Nucl Med 2006;47(6):1059- 1066.

[13] Robert A. Gatenby, MD,corresponding author Olya Grove,and Robert J. Gillies,"Quantitative Imaging in Cancer Evolution and Radiology". 2013 Oct; 269(1): 814 .

[14] Clarke LP, Nordstrom RJ, Zhang H, et al. “The Quantitative Imaging Network: NCI's Historical Perspective and Planned Goals". Transl Oncol 2014;7(1):1-4.

[15] Buckler AJ, Bresolin L, Dunnick NR, Sullivan DC; “. A collaborative enterprise for multistakeholder participation in the advancement of quantitative imaging”, Radiology 2011;258(3):906-914.

[16] Gatenby RA, Grove O, Gillies RJ. "Quantitative imaging in cancer evolution and ecology”. Radiology 2013;269(1):8-15.

[17] Parmar C, Rios Velazquez E, Leijenaar , Jermoumi M, Carvalho S, Mak RH, Mitra S, Shankar BU, Kikinis R, Haibe-Kains B , Lambin P, Aerts HJ." Robust Radiomics feature 
quantification using semiautomatic volumetric segmentation.” PLoS One. 2014 Jul 15;9(7):e102107. doi: 10.1371/journal.pone.0102107.

[18] Haralick RM, Shanmugam K, Dinstein I. “Textural features for image classification”. IEEE Trans Syst Man Cybern 1973;SMC- 3(6):610-621.

[19] Davnall F, Yip CS, Ljungqvist G, et al. "Assessment of tumor heterogeneity: an emerging imaging tool for clinical practice?" Insights Imaging 2012;3(6):573-589.

[20] O'Connor JP, Rose CJ, Waterton JC, Carano RA, Parker GJ, Jackson A. "Imaging intratumor heterogeneity: role in therapy response, resistance, and clinical outcome”. Clin Cancer Res 2015;21(2):249-257.

[21] Thompson, Anthony C. (1996). Minkowski Geometry. Encyclopedia of Mathematics and Its Applications. Cambridge University Press. ISBN 0-521-40472-X.

[22] Kumar V, Gu Y, Basu S, et al. "Radiomics:vthe process and the challenges", Magn ResonvImaging 2012;30(9):1234-1248.

[23] Aerts HJ, Velazquez ER, Leijenaar RT, et al. "Decoding tumour phenotype by noninvasive imaging using a quantitative Radiomics approach”, Nat Commun 2014;5:4006.

[24] Ravindra Patil, Geetha Mahadevaiah, and Andre Dekker. "An Approach Toward Automatic Classification of Tumor Histopathology of Non-Small Cell Lung Cancer Based on Radiomic Features". TOMOGRAPHY, December 2016, Volume 2, Issue 4:374377 DOI: 10.18383/j.tom.2016.00244

[25] Grossmann P, Grove O, El-Hachem N, et al. "Identification of molecular phenotypes in lung cancer by integrating Radiomics and genomics”. Sci Transl Med .

[26] Pickup L, Talwar A, Stalin S, et al. "Lung nodule classification using learnt texture features on a single patient population ", Radiological Society of North America Scientific Assembly and Annual Meeting Program. Oak Brook, Ill: Radiological Society of North America, 2015; SSMo6.

[27] Aerts, H. et al. Identification of residual metabolic-active areas within NSCLC tumours using a pre radiotherapy FDG-PET-CT scan: a prospective validation. Lung. Cancer. 75 , 73-76 (2012).

[28] Aerts, H. et al. Identification of residual metabolic-active areas within individual NSCLC tumours using a pre-radiotherapy (18)Fluorodeoxyglucose-PET-CT scan. Radiother. Oncol. 91, 386-92. (2009).

[29] Carrasco, J.L., King, T.S. \& Chinchilli, V.M. "The concordance correlation coefficient for repeated measures estimated by variance components." J Biopharm Stat 19, 90-105 (2009).

[30] Robert J. Gillies,Paul E. Kinahan, Hedvig Hricak, "Radiomics: Images Are More than Pictures, They Are Data.”, Radiology 2016,Volume 278, Issue 2

[31] Christopher M Florkowski, "Sensitivity, Specificity, Receiver-Operating Characteristic (ROC) Curves and Likelihood Ratios: Communicating the Performance of Diagnostic Tests" Clin Biochem Rev. 2008 Aug; 29(Suppl 1): S83-S87.

[32] Carrasco, J.L., Phillips, B.R., Puig-Martinez, J., King, T.S. \& Chinchilli, V.M. "Estimation of the concordance correlation coefficient for repeated measures using SAS and R". Comput Methods Programs Biomed 109, 293-304 (2013).

[33] Weimiao Wu, Chintan Parmar,Patrick Grossmann, John Quackenbush, Philippe Lambin,Johan Bussink,Raymond Mak and Hugo J. W. L. Aerts."Exploratory Study to Identify Radiomics Classifiers for Lung Cancer Histology" Front Oncol. 2016 Mar 30;6:71. doi: 10.3389/fonc.2016.00071. 
[34] Lin, L.I. "A concordance correlation coefficient to evaluate reproducibility". Biometrics 45, 255-68 (1989).

[35] Payam Refaeilzadeh, Lei Tang, Huan Liu. "Cross-Validation”. Encyclopedia of Database Systems, pp 532-538 (2009)

[36] Parmar C, Leijenaar RT, Grossmann P, et al. "Radiomic feature clusters and prognostic signatures specific for Lung and Head \& Neck cancer”. Sci Rep 2015;5:11044.

[37] Dhara AK, Mukhopadhyay S, Dutta A, et al. "A Combination of Shape and Texture Features for Classification of Pulmonary Nodules in Lung CT Images”. J Digit Imaging 2016;29:466-75.

[38] Dilger SK, Uthoff J, Judisch A, et al. "Improved pulmonary nodule classification utilizing quantitative lung parenchyma features.” J Med Imaging (Bellingham) 2015;2:041004.

[39] Andersen MB, Harders SW, Ganeshan B, Thygesen J, Torp Madsen HH, Rasmussen F, "CT texture analysis can help differentiate between malignant and benign lymph nodes in the mediastinum in patients suspected for lung cancer", Acta Radiol. 2016 Jun;57(6):669-76. doi: 10.1177/0284185115598808.

[40] Ganeshan B, Panayiotou E, Burnand K, et al. "Tumour heterogeneity in non-small cell lung carcinoma assessed by CT texture analysis: a potential marker of survival". Eur Radiol 2012;22:796-802.

[41] Ahn SY, Park CM, Park SJ, Kim HJ, Song C, Lee SM, McAdams HP, Goo JM. "Prognostic value of computed tomography texture features in non-small cell lung cancers treated with definitive concomitant chemoradiotherapy", Invest Radiol. 2015 Oct;50(10):719-25. doi: 10.1097/RLI.ooooooooooooo174.

[42] Hayano K, Kulkarni NM1, Duda DG, Heist RS, Sahani DV.” Exploration of Imaging Biomarkers for Predicting Survival of Patients With Advanced Non-Small Cell Lung Cancer Treated With Antiangiogenic Chemotherapy." AJR Am J Roentgenol. 2016 May;206(5):987-93. doi: 10.2214/AJR.15.15528.

[43] Balagurunathan Y, Gu Y, Wang H, et al. "Reproducibility and Prognosis of Quantitative Features Extracted from CT Images.”, Transl Oncol 2014;7:72-87.

[44] Carvalho S, Leijenaar RT, Velazquez ER, et al. "Prognostic value of metabolic metrics extracted from baseline positron emission tomography images in non-small cell lung cancer." Acta Oncol 2013;52:1398-404.

[45] Cook GJ, Yip C, Siddique M, et al. "Are pretreatment 18F-FDG PET tumor textural features in non-small cell lung cancer associated with response and survival after chemoradiotherapy?", J Nucl Med 2013;54:19-26.

[46] Fried DV, Mawlawi O, Zhang L, et al. "Stage III Non- Small Cell Lung Cancer: Prognostic Value of FDG PET Quantitative Imaging Features Combined with Clinical Prognostic Factors.", Radiology 2016;278:214-22.

[47] Coroller TP, Agrawal V, Narayan V, et al. "Radiomic phenotype features predict pathological response in nonsmall cell lung cancer.", Radiother Oncol 2016;119:480-6.

[48] Coroller TP, Grossmann P, Hou Y, et al. "CT-based radiomic signature predicts distant metastasis in lung adenocarcinoma." Radiother Oncol 2015;114:345-50.

[49] Miles KA, Cancer Imaging. 2016 Apr 11;16:10. doi: 10.1186/s40644-016-0065-5. How to use CT texture analysis for prognostication of non-small cell lung cancer.

[5o] Mattonen SA, Palma DA, Johnson C, et al. Detection of Local Cancer Recurrence After Stereotactic Ablative Radiation Therapy for Lung Cancer: Physician Performance Versus Radiomic Assessment. Int J Radiat Oncol Biol Phys 2016;94:1121-8. 
[51] Desseroit MC, Visvikis D, Tixier F, et al. "Development of a nomogram combining clinical staging with F-FDG PET/CT image features in non-small-cell lung cancer stage I-III.", Eur J Nucl Med Mol Imaging 2016;43:1477-85.

[52] Yoon HJ, Sohn I, Cho JH, et al. "Decoding Tumor Phenotypes for ALK, ROS1, and RET Fusions in Lung Adenocarcinoma Using a Radiomics Approach.”, Medicine (Baltimore) 2015;94:e1753.

[53] Leijenaar RT, Carvalho S, Velazquez ER, et al. "Stability of FDG-PET Radiomics features: an integrated analysis of test-retest and inter-observer variability",. Acta Oncol 2013;52:1391-7.

[54] Parmar C, Grossmann P, Bussink J, Lambin P, Aerts HJ."Machine Learning methods for Quantitative Radiomic Biomarkers.", Sci Rep. 2015 Aug 17;5:13087. doi: 10.1038/srep13087.

[55] Chalmers I, Glasziou P. "Avoidable waste in the production and reporting of research evidence”. Lancet 2009;374(9683):86-89.

[56] Simera I, Moher D, Hirst A, Hoey J, Schulz KF, Altman DG. “Transparent and accurate reporting increases reliability, utility, and impact of your research: reporting guidelines and the EQUATOR Network.", BMC Med 2010;8:24.

[57] Prinz F, Schlange T, Asadullah K. "Believe it or not: how much can we rely on published data on potential drug targets?", Nat Rev Drug Discov 2011;10(9):712.

[58] Gary S. Collins, Johannes B. Reitsma, Douglas G. Altman, Karel G.M. Moons."Transparent Reporting of a multivariable prediction model for Individual Prognosis Or Diagnosis (TRIPOD): The TRIPOD Statement, Annals of internal medicine “,Ann Intern Med. 2015;162(1):55-63. DOI: 10.7326/M14-0697

[59] Galavis PE, Hollensen C, Jallow N, et al. "Variability of textural features in FDG PET images due to different acquisition modes and reconstruction parameters.", Acta Oncol 2010;49:1012-6.

[6o] Yip S, McCall K, Aristophanous M, Chen AB, Aerts HJ, Berbeco R. "Comparison of texture features derived from static and respiratory-gated PET images in non-small cell lung cancer.", PLoS One. 2014 Dec 17;9(12):e115510. doi: 10.1371/journal.pone.0115510. eCollection 2014.

[61] Fave X, Cook M, Frederick A, Zhang L, Yang J, Fried D, Stingo F, Court L. "Preliminary investigation into sources of uncertainty in quantitative imaging features.", Comput Med Imaging Graph. 2015 Sep;44:54-61. doi: 10.1016/j.compmedimag.2015.04.oo6. Epub 2015 May 5.

[62] Fave X, Mackin D, Yang J, et al. "Can Radiomics features be reproducibly measured from CBCT images for patients with non-small cell lung cancer?”, Med Phys 2015;42:6784-97.

[63] Scrivener M, de Jong EEC, van Timmeren JE, Pieters T, Ghaye B, Geets X. Radiomics applied to lung cancer: a review. Translational Cancer Research 2016;5: 398-409

[64] Yip SS, Aerts HJ. Applications and limitations of Radiomics. Phys Med Biol 2016;61: R15 o-166.

[65] Larue RT, Defraene G, De Ruysscher D, Lambin P, van Elmpt W. Quantitative Radiomi cs studies for tissue

characterization: a review of technology and methodological procedures. Br J Radiol 20 17;90: 20160665 .

[66] Alberto Traverso et.al, Repeatability and Reproducibility of Radiomic Features: A Systematic Review, International journal of Radiation Oncology, International of Radiation Oncology 2018, vol 102 , issue 4 
[67] Cusumano D et.al, Fractal-based radiomic approach to predict complete pathological response after chemo-radiotherapy in rectal cancer, Radiol Med. 2018 Apr; 123(4):286295. doi: 10.1007/s11547-017-0838-3. Epub 2017 Dec 11.

[68] Deist TM,Machine learning algorithms for outcome prediction in (chemo)radiotherapy: An empirical comparison of classifiers, Med Phys. 2018 Jul;45(7):3449-3459. doi: 10.1002/mp.12967. Epub 2018 Jun 13.

[69] Jake Lever, Martin Krzywinski \& Naomi Altman, Model selection and overfitting, Nature Methods volume13, pages703-704 (2016)

[7o] Haynes, R. B. et al. Optimal search strategies for retrieving scientifically strong studies of treatment from Medline: analytical survey. BMJ 330, 1179 (2005).

[71] Ingui, B. J. \& Rogers, M. A. Searching for clinical prediction rules in MEDLINE. J. Am. Med. Inform. Assoc. JAMIA 8, 391-397 (2001).

[72] Geersing, G.-J. et al. Search filters for finding prognostic and diagnostic prediction studies in Medline to enhance systematic reviews. PloS One 7, e32844 (2012).

[73] Cheng N-M, Fang Y-HD, Tsan D-L, Hsu CH, Yen T-C (2016) Respiration-Averaged CT for Attenuation Correction of PET Images - Impact on PET Texture Features in NonSmall Cell Lung Cancer Patients. PLoS ONE 11(3): e0150509. doi:10.1371/ journal.pone.0150509

[74] David V. Fried, BS, Susan L. Tucker, Shouhao Zhou, Zhongxing Liao, Prognostic Value and Reproducibility of Pretreatment CT Texture Features in Stage III Non-Small Cell Lung Cancer, Int J Radiat Oncol Biol Phys. 2014 November 15; 9o(4): 834-842. doi:10.1016/j.ijrobp.2014.07.020

[75] David V. Fried, BS,Osama Mawlawi, Lifei Zhang, Xenia Fave, BS, Shouhao Zhou, Geoffrey Ibbott, Zhongxing Liao and Laurence E. Court ,Potential Use of 18Ffluorodeoxyglucose Positron Emission Tomography Based Quantitative Imaging Features for Guiding Dose Escalation in Stage III Non-Small Cell Lung Cancer, Int J Radiation Oncol Biol Phys, Vol. 94, No. 2, pp. 368e376, 2016 0360-3016/\$

[76] Pierre Lovinfosse, Zsolt Levente Janvary, Philippe Coucke, FDG PET/CT texture analysis for predicting the outcome of lung cancer treated by stereotactic body radiation therapy, Eur J Nucl Med Mol Imaging (2016) 43:1453-1460

[77] Nitin Ohri, Fenghai Duan, Bradley S. Snyder, Bo Wei, Mitchell Machtay, Abass Alavi, Barry A. Siegel, Douglas W. Johnson, Jeffrey D. Bradley, Albert DeNittis, Maria WernerWasik, and Issam El Naqa Pretreatment 18F-FDG PET Textural Features in Locally Advanced Non-Small Cell Lung Cancer: Secondary Analysis of ACRIN 6668/RTOG 0235, Nucl Med. 2016 June ; 57(6): 842-848. doi:10.2967/jnumed.115.166934.

[78] Pyka et al. , Textural features in pre-treatment [F18]-FDG-PET/CT are correlated with risk of local recurrence and disease-specific survival in early stage NSCLC patients receiving primary stereotactic radiation therapy; Radiation Oncology (2015) 10:100 DOI 10.1186/s13014-015-0407-7

[79] Jia Wu et.al Early-Stage Non-Small Cell Lung Cancer: Quantitative Imaging Characteristics of $18 \mathrm{~F}$ Fluorodeoxyglucose PET/CT Allow Prediction of Distant Metastasis1, Radiology: Volume 281: Number 1-October 2016

[8o] Elizabeth Huynh, Thibaud P. Coroller, Vivek Narayan, CT-based radiomic analysis of stereotactic body radiation therapy patients with lung cancer; Radiotherapy and Oncology 120 (2016) 258-266 
[81] Manushka Vaidya, Kimberly M. Creach , Jennifer Frye, Farrokh Dehdashti, Jeffrey D. Bradley, Issam El Naqa; Combined PET/CT image characteristics for radiotherapy tumor response in lung cancer, Radiotherapy and Oncology 102 (2012) 239-245

[82] Thida Win, Tumor Heterogeneity and Permeability as Measured on the CT Component of PET/CT Predict Survival in Patients with Non-Small Cell Lung Cancer, Imaging, Diagnosis, Prognosis, DOI: 10.1158/1078-0432.CCR-12-1307

[83] Heyse Li, The value of nodal information in predicting lung cancer relapse using 4DPET/4DCT, American Association of Physicists in Medicine. [http://dx.doi.org/10.1118/1.4926755]

[84] Yanqi Huang et al, Radiomics Signature: A Potential Biomarker for the Prediction of Disease-Free Survival in Early-Stage (I or II) Non-Small Cell Lung Cancerı, Radiology: Volume 281: Number 3-December 2016

[85] Gary J. R. Cook et al, Non-Small Cell Lung Cancer Treated with Erlotinib: Heterogeneity of 18F-FDG Uptake at PET-Association with Treatment Response and Prognosis, Radiology: Volume 276: Number 3-September 2015

[86] Dong X, Sun X, Sun L, Maxim PG, Xing L,Huang Y, et al. (2016) Early Change in Metabolic Tumor Heterogeneity during Chemoradiotherapy and Its Prognostic Value for Patients with Locally Advanced Non-Small Cell Lung Cancer. PLoS ONE 11(6): eo157836. doi:10.1371/journal.pone.0157836

[87] Mattea L.Welch et.al, Vulnerabilities of radiomic signature development: The need for safeguards panel , Radiotherapy and Oncology Volume 130, January 2019, Pages 2-9

[88] C Parmar, ER Velazquez, R Leijenaar, M Jermoumi, S Carvalho, RH Mak Robust Radiomics feature quantification using semiautomatic volumetric segmentation, ...PloS one 9 ( 7$)$, e102107 


\section{Chapter 3}

\section{Deep Learning based Auto Contouring of the Lung}

Ravindra Patil, Leonard Wee and Andre Dekker

Auto Segmentation of Lung in Non-small Cell Lung Cancer Using Deep Convolution Neural Network. Advances in Computing and Data Sciences. ICACDS. Communications in Computer and Information Science, 2020, vol 1244. Springer, DOI: $\mathrm{https}$ ://doi.org/10.1007/978981-15-6634-9-31 


\begin{abstract}
Segmentation of the lung is the vital first step in the radiologic diagnosis of lung cancer. In this work, we present a deep learning based automated technique that overcomes various shortcomings of traditional lung segmentation and explores the role of adding "explainability" to deep learning models for enhanced trust in these models. Our approach shows better generalization across different scanner settings, vendors and slice thickness. In addition, there is no initialization of the seed point needed, making it completely automated without manual intervention. The dice score of 0.98 is achieved for lung segmentation on an independent data set of non-small cell lung cancer patients.
\end{abstract}

Keywords : Segmentation, Deep Learning, Lung 


\section{Introduction}

Lung cancer is the leading cause of cancer related deaths and carries a dismal prognosis with a 5-year survival rate at only $18 \%$ [1]. Out of all lung cancers, Non-Small Cell Lung Cancer (NSCLC) accounts for $85 \%$ of the cases. Treatment monitoring and analysis [2] using computed tomography (CT) images are important strategies for early lung cancer diagnosis and survival time improvement. In these approaches, accurate lung anatomy and pathology region segmentation is necessary as it directly relates to the treatment plan. After decades of development in imaging techniques, volumes of high-resolution images with low distortions are now more easily available. Despite the development of approaches for lung segmentation in recent years [3-6], achieving accurate segmentation performance continues to require attention because of specific challenges. One such example is tumors have an intensity similar to that of the lung wall; thus, they are difficult to distinguish using intensity values alone and also the structure of the lung changes based on the disease pathology such as consolidation, masses, atelectasis, pneumothorax or effusions.

There is considerable progress in development of lung segmentation algorithms which have the ability to perform accurate delineations under different diseases conditions [7]. In general, lung segmentation algorithms can be classified into one of the following five sub categories (1) Intensity based (2) Shape based or Model based (3) Neighboring anatomy guided (4) Region based (5) Artificial Intelligence based [7]. The intensity based approaches are fast, intuitive and computationally efficient however it fails during the pathological condition where there are limited attenuation variations. Shape or model based approaches provide a very good accuracy due to anatomical modelling and template mapping, however these algorithms are computationally inefficient and it has proven difficult to create representative training features. Neighboring anatomy based approach exploits the information of the spatial context of the neighboring organ of the lung such as the rib cage, heart, spine for extracting the contours of the lung region, this approach is computationally expensive but provides good results when the intensity 
variation is mild to moderate. However, in case of more extreme disease conditions such as opacification of the entire hemithorax, this approach fails. Region growing approaches such as watershed transforms, graph cuts and random walks are efficient but they tend to over segment. In recent times, AI based (Machine Leaning and Deep Learning ) approaches have become popular and in particular Deep Learning (DL) approaches due to the better accuracy that these algorithms achieve in varying pathologic conditions [8].

Recently, Convolutional Neural Networks (CNN) have been seen as a powerful tool for learning features from network layers [9]. CNN's act as a tool for learning discriminative features, which are useful in different image processing and computer vision tasks. CNN's need relatively less pre-processing compared to other known algorithms, which means the network learns the filters that in traditional algorithms are hand engineered [9]. The independence from prior knowledge and effort required in feature selection design is a major advantage. Moreover, CNN's include multi-layer processing, which ensures that the model learns the features at the granular level. In recent time, there has been progressive usage of CNN's for various medical segmentation tasks. Dou et.al [10] uses a $3 \mathrm{D} C N N$, which focuses on the task of automatic nodule detection. DIAG Convnet by Setio et.al [11] provides automatic pulmonary nodule detection in CT images. The review article by Zhou et.al [12] lists various deep learning based medical segmentation approaches developed for different modalities and anatomies. Although, CNN's are explored for medical image segmentation, we did not come across a study with large-scale validation of for lung segmentation with deep learning in diseased conditions.

The current work has several objectives. The first is to test the efficacy of the deep learning models on a large-scale lung cancer dataset to automatically segment the lung region. Second, to compare the deep learning approach with the traditional segmentation methods and comment on the complexity and efficiency. Third, adding "explainability" to deep learning models so that trust can be enhanced for these models. 


\section{Methodology}

In the traditional approach of lung segmentation, the canonical steps are employed as shown in Figure 1. However, there might be a slight variation in each of the blocks based on the algorithm that is considered for implementation. Each of these blocks needs tuning based on the image type and the acquisition parameters of the scanner. Marker generation is used to define the region that is present inside and outside the lung region; this is performed manually by marking the region using a seed point. Further, the image is preprocessed by applying the filters as well as Hounsfield unit (HU) thresholding to eliminate unwanted region. This is followed by running a segmenter algorithm (such as watershed, active contour etc.. ) and performing the morphological and post processing operations to correct the contours. These traditional approaches need manual and empirical tuning and are quite difficult to generalize on large and varied datasets with different acquisitions and threshold values.

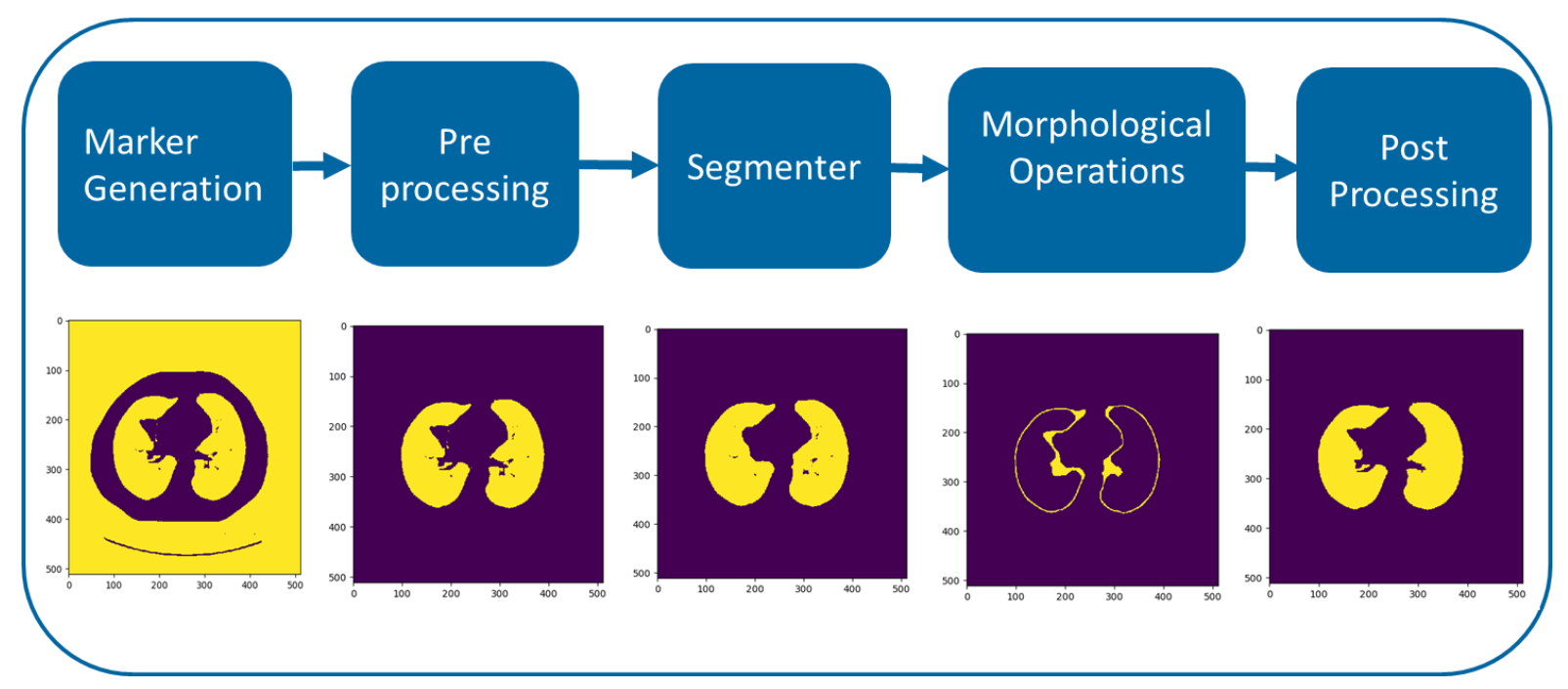

Figure 1. Traditional lung segmentation Approach

The approach adapted in this work is described in the section below, which is represented by the block diagram schematic shown in the Figure 2. There are two phases in creation of the deep learning model. First is the training phase, data is fed after preprocessing to the model and the model is trained with the annotated ground 
truth (region of the lung) defined by RT Structure (RTSTRUCT) as reference. Further, the learnt model layers are analyzed using visualization to ascertain, what region of the image the model looked into to arrive at the delineation of the lung region. In the scoring phase, the trained model is used to delineate the region of the lung on unseen lung cancer CT scans.

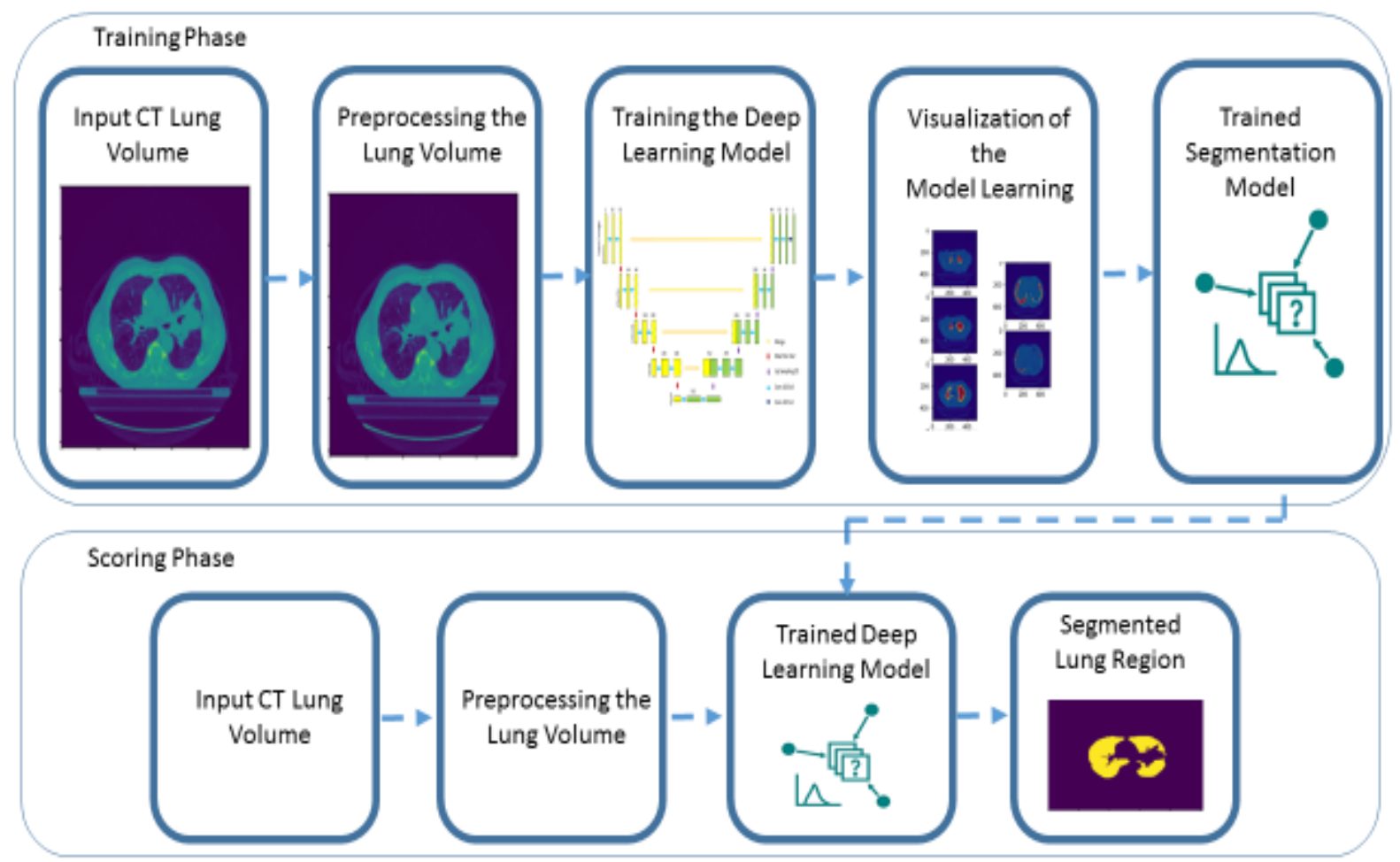

Figure 2. Deep Learning workflow of Lung segmentation

\section{Data:}

The data set used for model training which was obtained from a The Cancer Imaging Archive (TCIA) repository of NSCLC patients [13]. This dataset contained pretreatment CT scans with manually delineated lung regions by the radiation oncologist. This data set will be referred to as Lung 1. In total 422 subjects were used for training, which maps to $\sim 42$, 019 lung CT slices on which the algorithm was trained. Out of 422 subjects, 300 subjects were randomly chosen for model training and the remaining 122 for model validation. The training data had the following sub categories of NSCLC: adenocarcinoma, large cell carcinoma and squamous cell carcinoma. The CT scanners used for imaging the subjects were from different vendors (Siemens, CNS Inc, Philips and GE) and the slice thickness varied from 0.65 to $5 \mathrm{~mm}$ with 512 * 512 
resolution. Furthermore, for testing the model an independent data set of Lung 2 was used which is marked as the NSCLC Radiogenomics dataset in a TCIA repository with 211 subject's [14]. The testing data had the following different sub categories of NSCLC: Adenocarcinoma, Squamous cell carcinoma and NOS. The demographics of each of the subgroups of training and testing are mentioned in the Figure 3.

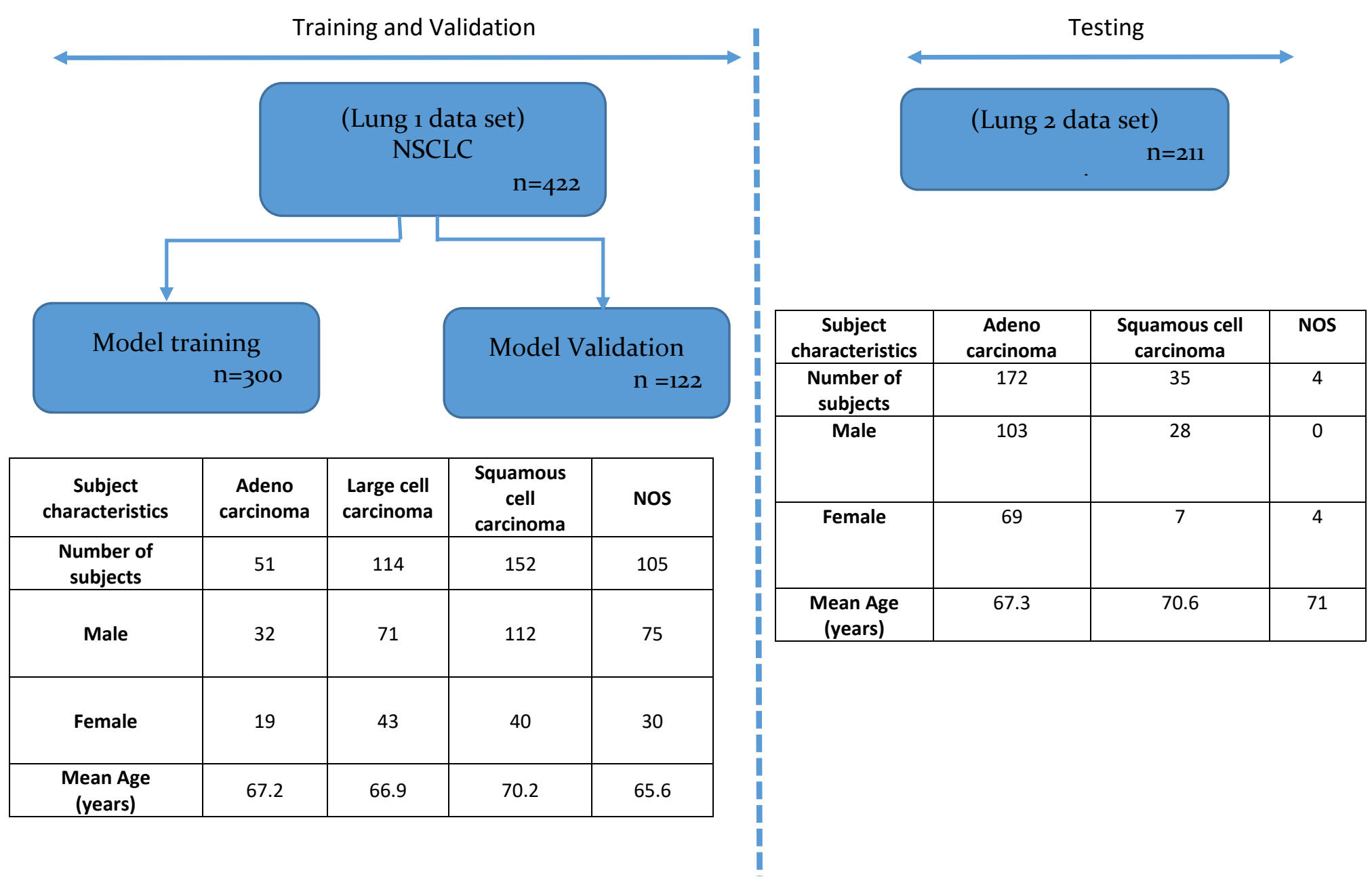

Figure 3. Data Demographics and Data Split for model training

\section{Pre processing}

The input CT volumes are processed to set non-anatomical regions such as air (with HU value below -100o) to o, so that the number of the pixel computations are reduced. Data augmentation was performed in terms of translation and rotation (o to 30 degrees) to make the model more robust against data variations. No specific preprocessing steps 
such as de-noising, artifact corrections, system-based calibration were employed, which are typical in conventional approaches of lung segmentation. The preprocessing approach was specifically designed to have a minimum number of steps, to test the efficacy of convolution neural networks.

\section{Training Deep Learning Model}

The target of the current approach is to segment the lung given a CT slice which contains a (diseased or non-diseased) lung. We employ a modified U-Net [15] inspired CNN architecture to arrive at a lung segmentation. We follow a pixel based classification mechanism that aims at classifying whether a pixel belongs to the lung or not. The model architecture that is built was inspired from U-Net Convolution Neural Network (CNN), which consists of 18 convolutional layers, 4 central pooling layers with the convolutional kernel size of $3 \mathrm{X}_{3}$ in each convolutional layer. The schematic representation of the model architecture is shown in Figure 4.

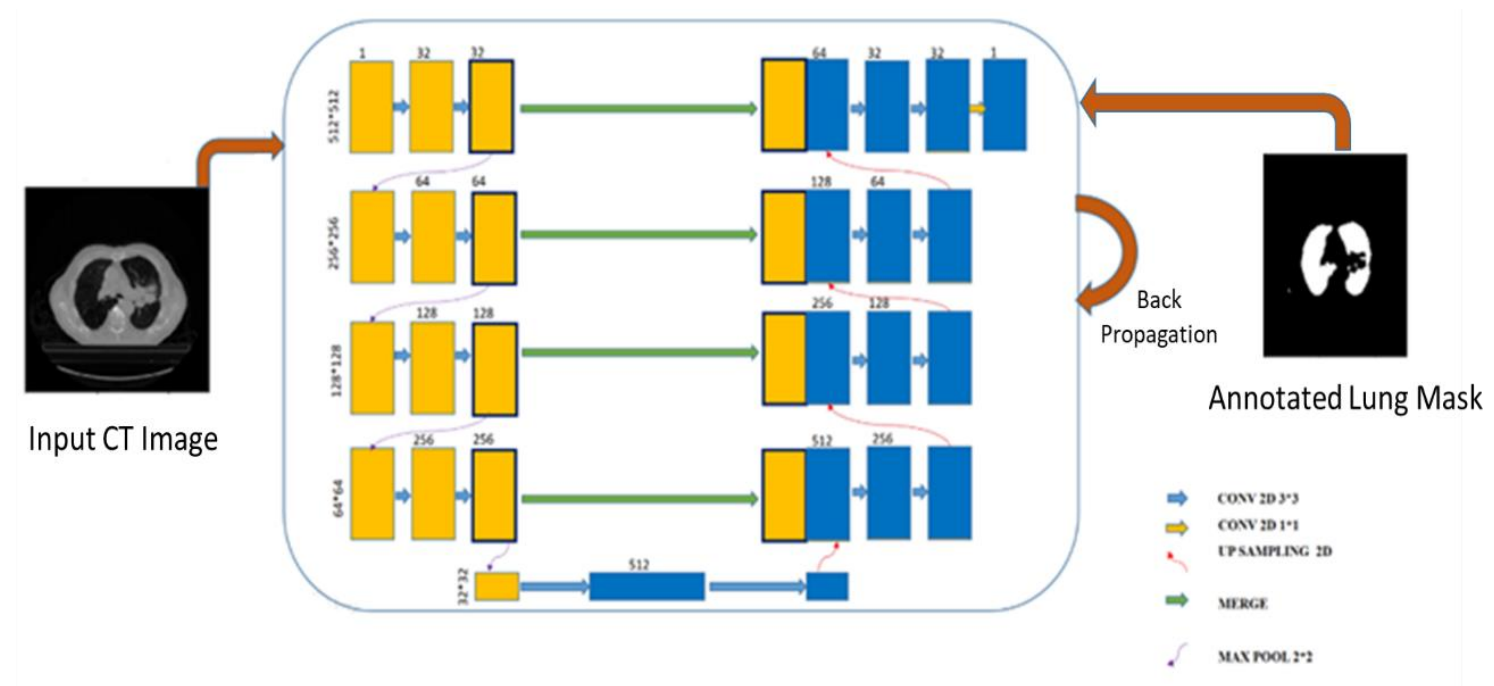

Figure 4. Model Architecture

The convolutional layers perform a convolutional operation on all input feature maps to obtain output features defined by the Rectified Linear Units (ReLU) activation function [16]. The feature map layer combination is defined by the equation (1) 


$$
f^{j}=\operatorname{ReLU}\left(\sum_{i=1}^{m} C^{i j} * f^{i}+b^{j}\right)
$$

Where $f^{i}$ and $f^{j}$ are the $i^{\text {th }}$ input feature map and $j^{\text {th }}$ output feature map, respectively. We define $C^{i j}$ as the convolutional kernel between $f^{i}$ and $f^{j} \quad{ }^{*}$ denotes the 2-D convolutional operation), $\mathrm{b}^{\mathrm{j}}$ is the bias of the $j^{\text {th }}$ output feature map.

After each convolutional layer, a rectified linear unit (ReLU) is used as a non-linear activation function, this is added to bring non linearity to the model and is expressed as:

$$
\operatorname{ReLU}(z)=\max (0, z)
$$

Further to the last convolutional layer, a fully connected layer is applied where each output unit connects to all inputs. This layer can capture correlations between different features produced by the convolutional layer. For achieving non-linearity and a twoclass output classifier, the sigmoid function was used. Since the sigmoid function ranges from zero to one, it can be directly related to class probabilities making it an ideal activation function for a classification task.

$$
\operatorname{sigmoid}(z)=\frac{1}{1+e^{-z}}
$$

The goal of network training is to maximize the probability of the correct class. This is achieved by minimizing the dice coefficient loss function. The loss function is minimized during the model's training process. The weight update was performed using Adaptive Moment Estimation (ADAM) algorithm [17]. Instead of adapting the parameter learning rates based on the average first moment as in RMSProp [18], ADAM makes use of the average of second moments of the gradients. Specifically, the algorithm calculates an exponential moving average of the gradient and the squared gradient, and the parameters $\beta_{1}, \beta_{2}$ control the decay rates of these moving averages. The initial value of the moving averages and $\beta_{1}, \beta_{2}$ values close to 1.0 (recommended) results in a bias of moment estimates towards zero. This bias is overcome by calculating the biased estimates and then calculating bias-corrected estimates. ADAM is an extension to stochastic gradient descent and converges faster than other stochastic optimization 
methods [18]. It also rectifies problems such as a vanishing learning rate and slow convergence that other optimization approaches face which leads to a fluctuating loss function. The weights are updated based on the below equation

$$
w^{(t+1)} \leftarrow w^{(t)}-\eta \frac{\widehat{w_{w}}}{\sqrt{v_{w}}+\varepsilon}
$$

Where $\varepsilon$ is a small number used to prevent division by zero.

And

$$
\begin{gathered}
\widehat{m_{w}}=\frac{m_{w}^{(t+1)}}{1-\beta_{1}^{t}} \\
\widehat{v_{w}}=\frac{v_{w}^{(t+1)}}{1-\beta_{2}^{t}}
\end{gathered}
$$

Where $m_{w}$ and $v_{w}$ are estimates of the first moment and second moment of the gradients respectively. $\beta_{1}$ and $\beta_{2}$ are the forgetting factors for gradients and second moments of gradients, respectively.

The layer parameters used in the above model is as below

\begin{tabular}{|l|l|l|l|}
\hline \multicolumn{1}{|c|}{ Layer } & \multicolumn{1}{|c|}{ Filters } & \multicolumn{1}{c|}{ Kernel Size } & \multicolumn{1}{c|}{$\begin{array}{c}\text { Activation } \\
\text { Function }\end{array}$} \\
\hline \multicolumn{1}{|c|}{ Step I } & & & \\
\hline $\begin{array}{l}\text { Convolution Layer } \\
1\end{array}$ & 32 & $3^{*} 3$ & ReLu \\
\hline $\begin{array}{l}\text { Convolution Layer } \\
2\end{array}$ & 32 & $3^{*} 3$ & ReLu \\
\hline Maxpooling Layer 3 & & $2^{*} 2$ & \\
\hline Step II & & & ReLu \\
\hline $\begin{array}{l}\text { Convolution Layer } \\
4\end{array}$ & 64 & $3^{*} 3$ & ReLu \\
\hline $\begin{array}{l}\text { Convolution Layer } \\
5\end{array}$ & 64 & $3^{*} 3$ & \\
\hline Maxpooling Layer 6 & & $2^{*} 2$ & \\
\hline & & & \\
\hline
\end{tabular}

70 | Prognostic and Prediction Modelling with Radiomics for Non-Small Cell Lung Cancer 


\begin{tabular}{|c|c|c|c|}
\hline Step III & & & \\
\hline $\begin{array}{l}\text { Convolution Layer } \\
7\end{array}$ & 128 & $3^{*} 3$ & ReLu \\
\hline $\begin{array}{l}\text { Convolution Layer } \\
8\end{array}$ & 128 & $3^{*} 3$ & ReLu \\
\hline Maxpooling Layer 9 & & $2 * 2$ & \\
\hline \multicolumn{4}{|l|}{ Step IV } \\
\hline $\begin{array}{l}\text { Convolution Layer } \\
10\end{array}$ & 256 & $3^{*} 3$ & ReLu \\
\hline $\begin{array}{l}\text { Convolution Layer } \\
11\end{array}$ & 256 & $3^{*} 3$ & ReLu \\
\hline $\begin{array}{l}\text { Maxpooling Layer } \\
12\end{array}$ & & $2^{*} 2$ & \\
\hline \multicolumn{4}{|l|}{ Step V } \\
\hline $\begin{array}{l}\text { Convolution Layer } \\
13\end{array}$ & 512 & $3^{*} 3$ & ReLu \\
\hline $\begin{array}{l}\text { Convolution Layer } \\
14\end{array}$ & 512 & $3^{*} 3$ & ReLu \\
\hline \multicolumn{4}{|l|}{ Step VI } \\
\hline $\begin{array}{l}\text { Upsampling Layer } \\
15\end{array}$ & & $2 * 2$ & \\
\hline $\begin{array}{l}\text { Convolution Layer } \\
16\end{array}$ & 256 & $3^{*} 3$ & ReLu \\
\hline $\begin{array}{l}\text { Convolution Layer } \\
17\end{array}$ & 256 & $3^{*} 3$ & ReLu \\
\hline \multicolumn{4}{|l|}{ Step VII } \\
\hline $\begin{array}{l}\text { Upsampling Layer } \\
18\end{array}$ & & $2^{*} 2$ & \\
\hline $\begin{array}{l}\text { Convolution Layer } \\
19\end{array}$ & 128 & $3^{*} 3$ & ReLu \\
\hline $\begin{array}{l}\text { Convolution Layer } \\
20\end{array}$ & 128 & $3^{*} 3$ & ReLu \\
\hline \multicolumn{4}{|l|}{ Step VIII } \\
\hline $\begin{array}{l}\text { Upsampling Layer } \\
21\end{array}$ & & $2^{*} 2$ & \\
\hline
\end{tabular}

Prognostic and Prediction Modelling with Radiomics for Non-Small Cell Lung Cancer $\mid 71$ 


\begin{tabular}{|c|c|c|c|}
\hline $\begin{array}{l}\text { Convolution Layer } \\
22\end{array}$ & 64 & $3 * 3$ & ReLu \\
\hline $\begin{array}{l}\text { Convolution Layer } \\
23\end{array}$ & 64 & $3 * 3$ & ReLu \\
\hline \multicolumn{4}{|l|}{ Step IX } \\
\hline $\begin{array}{l}\text { Upsampling Layer } \\
24\end{array}$ & & $2^{*} 2$ & \\
\hline $\begin{array}{l}\text { Convolution Layer } \\
25\end{array}$ & 32 & $3^{*} 3$ & ReLu \\
\hline $\begin{array}{l}\text { Convolution Layer } \\
26\end{array}$ & 32 & $3^{*} 3$ & ReLu \\
\hline Output Layer & 1 & $1^{*} 1$ & Sigmoid \\
\hline
\end{tabular}

The hyperparameters used for the model training are Optimizer = ADAM, Learning Rate=1.oe-6, Metric $=$ Dice score, Number of Epochs $=50$, Batch Size $=2$, Weight Initialization Method = Xavier initialization. The model was built using Keras (2.1.1) and Tensor flow (1.2.1) as backend in python 3.o. The machine configuration used for training the model had Nvidia GPU (Titan X 1080Ti) with the mentioned model hyper parameters. The dice similarity coefficient (DSC) is used as the primary evaluation criteria for assessing the automatic segmentation accuracy; also, this is used as a loss function for the backward propagation in the proposed model. The DSC expressed as in equation (7) provides an amount of overlap between two segmentation results [19], wherein $G_{t}$ is the ground truth segmentation and Auto is the automated segmentation performed by the trained model.

$$
D S C=\frac{2 * V\left(G_{t} \cap \text { Auto }\right)}{V\left(G_{t}\right)+V(\text { Auto })}
$$

In the implementation, the RTSTRUCT containing the lung segmentation by the radiologist is considered as the ground truth and the region predicted by the model is overlaid on the RTSTRUCT mask to arrive at the DSC.

\section{Model Visualization}


Interpreting the deep learning model and understanding the model's rationale behind the decision process to arrive at the prediction is challenging. In order to ensure that the model is indeed looking into the relevant regions in the input image to arrive at the decision, a visualization engine was added on top of the built model. The approach used to build the model visualization was Gradient-weighted Class Activation Mapping (Grad-CAM) [20]. The Grad-CAM works on the class discriminative localization approach wherein it uses the gradients of the target of the final convolutional layer to produce a coarse localization map, highlighting the regions considered in the image for predicting the concept. This is not only useful in order to know about the regions responsible for the prediction but also aids in debugging the decision process in the networks. In essence, Grad-CAM takes into account the penultimate layer (layer before softmax) to interpret the decision of the $\mathrm{CNN}$ and identifies the respective filter activation for every spatial location $(\mathrm{i}, \mathrm{j})$ in the given image. Then this is converted into the heat map based on the weights indicating the prominent regions. The mathematical aspect is depicted in the below equations 8 and 9 .

$$
\begin{aligned}
& W_{k}^{c}=\frac{1}{z} \sum_{i} \sum_{j} \frac{\partial Y^{c}}{\partial A_{i j}^{k}} \\
& S^{c}=\frac{1}{z} \sum_{i} \sum_{j} \sum_{k} W_{k}^{c} A_{i j}^{k}
\end{aligned}
$$

The spatial score of a specific class $S^{c}$ is the global average pooling over spatial location $(i, j)$ for the gradient of respective class output $Y^{c}$ with respect to the feature map $A_{i j}^{k}$. The spatial score is obtained by multiplying the resulting value with the feature map along with its channel axis $k$. The $\sum$ describes the pooling and average operation and $z$ is constant. The output of the sample GradCAM results can be seen in Figure 5. It can be observed from the figure that some locations in the lungs are highlighted with a red color indicating the maximum activation of the filters in that region, leading to the understanding that the model has indeed focused on the correct region of the image for lung segmentation. 

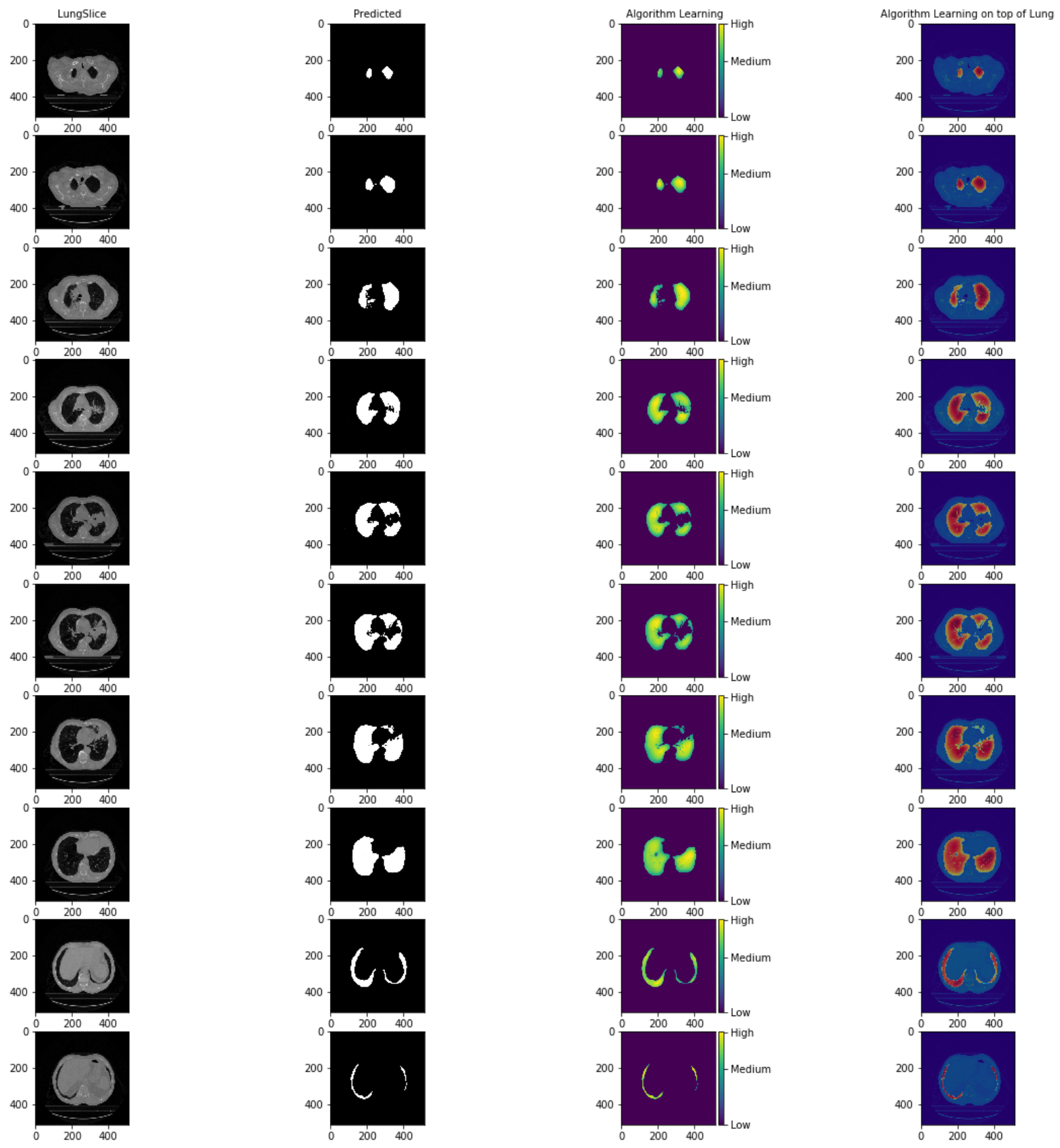

Figure 5. GradCAM visualization on the Lung region.

\section{Results and Discussion:}

A training accuracy of 0.99 dice score was obtained after 50 epochs of training. On the independent test data of Lung 2 dataset an average dice score of 0.98 was obtained. Also, the box plot in the Figure 6 on the test data shows the similar results indicating the average predicted dice score in the range of 1 to 0.98 without outliers being seen, thus suggesting the robustness of the model. The scoring time on the same GPU configuration took one millisecond per lung slice. 


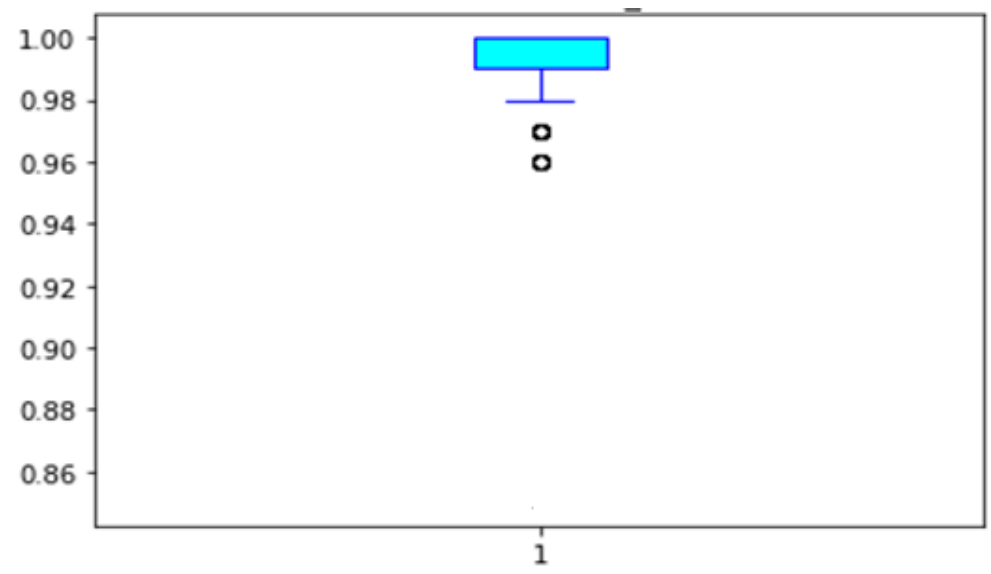

Figure 6. Box Plot of Dice Score of Lung anatomy on the Test dataset 

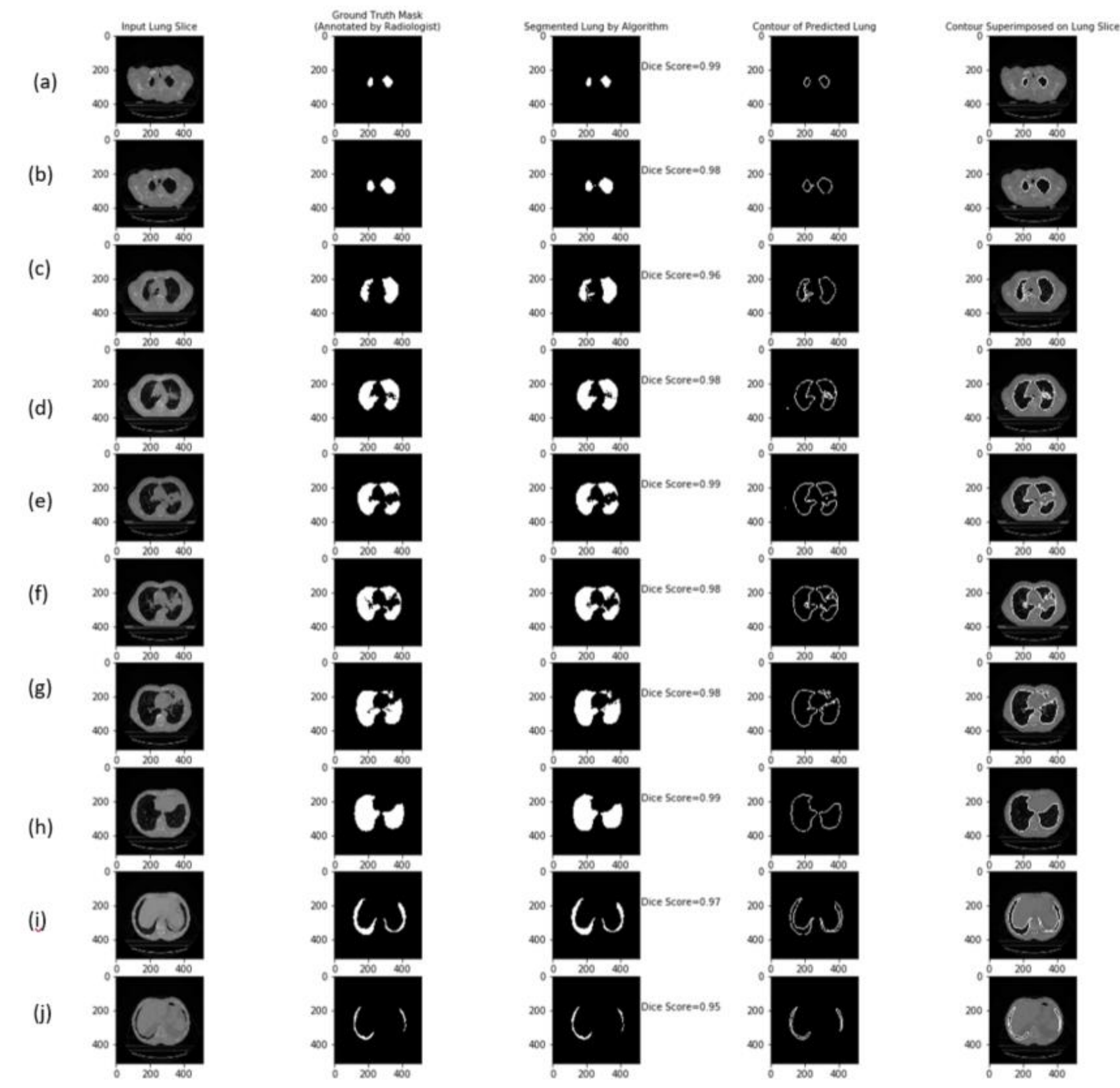

Figure 7. Segmented Lung region and the associated ground truth masks

The sample results of segmented output can be observed in Figure 7. The first column is input CT slice, the second column depicts annotation of the lung marked by the radiation oncologist, the third column is the segmented output by our model, and the fourth is the manual lung contour subtracted from the model based segmentation and fifth column is this subtraction overlaid on the original lung slice. It can be observed that the lung segmentation model is robust to the anatomical structures of the lung ranging from symmetric lungs (rows d-f) to only partially visible lung regions in the bottom two rows of the figure. 


\section{Conclusion and Comparison with traditional approaches}

Multiple algorithms in the literature have attempted segmentation of the lung with algorithms varying from simple thresholding to machine learning approaches. Table 3 provides a comparison of recently published lung segmentation approaches.

\begin{tabular}{|c|c|c|c|c|}
\hline Title & Approach & $\begin{array}{c}\text { Number of } \\
\text { dataset (n) }\end{array}$ & $\begin{array}{c}\text { Average } \\
\text { Reported } \\
\text { Dice Score }\end{array}$ & $\begin{array}{c}\text { External } \\
\text { Dataset }\end{array}$ \\
\hline $\begin{array}{c}\text { Y.Wei,G et.al } \\
\text { [21] }\end{array}$ & $\begin{array}{c}\text { Bresenham } \\
\text { algorithm }\end{array}$ & 97 subjects & 0.95 & $\begin{array}{c}\text { Externally } \\
\text { validated on } \\
25 \text { Subjects }\end{array}$ \\
\hline Dai et.al [22] & $\begin{array}{c}\text { Gaussian Mixture } \\
\text { based model }\end{array}$ & Not mentioned & 0.98 & $\begin{array}{c}\text { No external } \\
\text { Validation }\end{array}$ \\
\hline Noor et.al [23] & $\begin{array}{c}\text { Thresholding and } \\
\text { Morphological } \\
\text { operation }\end{array}$ & 96 subjects & 0.98 & $\begin{array}{c}\text { No external } \\
\text { validation }\end{array}$ \\
\hline Zhang et al [24] & $\begin{array}{c}\text { Active Contour } \\
\text { based approach }\end{array}$ & 60 subjects & 0.97 & $\begin{array}{c}\text { Externally } \\
\text { validated on } \\
6 \text { subjects }\end{array}$ \\
\hline Our Approach & Deep Learning & 422 Subjects & 0.98 & $\begin{array}{c}\text { Externally } \\
\text { validated on } \\
\text { 211 subjects }\end{array}$ \\
\hline
\end{tabular}

Table 3. Comparison with other Lung Segmentation Approaches

Prior work by Y.Wei et.al [21] performs both preprocessing as well as post processing of the scans to arrive at the segmentation. It was validated on a limited external data of 25 subjects leading to a DSC of 0.95 . However, as there are preprocessing steps used in this study, where each of these steps needs to be optimized based on the scan type, slice thickness and acquisition parameters the generalization of the approach is limited. Another study by Dai et.al [22] used a Gaussian mixture model based approach. In this, there is a manual seed point is needed as well as initialization and custom preprocessing for noise elimination. The study does not mention the data characteristics or the diseases under consideration or the number of subjects used for building the model, limiting the evaluation of the claims of the study. Noor et.al [23] used a thresholding approach and claimed a DSC of 0.98 without an external validation data set. In addition, the study focused only on a single scanner type as well as a single slice thickness of 10 mm making the approach difficult to generalize across all scanner types . Another study by Zhang et.al [24] showed the robustness of an active contour model for delineation of the lung with pathological conditions. Also the model took into account the variability of scanner setting 
and the data. Furthermore, the model was validated on an external dataset of 60 subjects resulting in a dice score of 0.97 .. However, in this study there is a need to initialize the threshold value and perform preprocessing steps such as Gaussian smoothing. Compared to existing approaches, our model is exhaustively validated on a external, diseased data set and there is no preprocessing or post processing performed andno domain-based adaptation is performed to arrive at the delineation. Our approach is expected to generalize well across different scanner settings, vendors and the slice thickness. In addition, there is no initialization or seed point needed making it a completely automated solution needing no manual intervention. 


\section{Reference}

[1] Siegel, R. , Miller, K. , Jemal, A. , 2016. Cancer statistics, 2016. CA-Cancer J. Clin. 66, 7-30 .

[2] Aerts, H. , Velazquez, E. , Leijenaar, R. , Parmar, C. , Grossmann, P. , Carvalho, S. , et al. , 2014. Decoding tumour phenotype by noninvasive imaging using a quantitative Radiomics approach. Nat Commun. 2014 Jun 3;5:4006. doi: 10.1038/ncomms50o6.

[3] Y.Wei,G.Shen,andJ.J.Li,"A fully automatic method for lung parenchyma segmentation and repairing,"J. Digit. Imaging, vol.26, no.3, pp. 483-495, 2013.

[4] S.Dai,K.Lu,J.Dong,Y.Zhang, and Y.Chen, "A novel approach of lung segmentation on chest CT images using graph cuts,"Neurocomputing,vol. 168, pp. 799-807, 2015.

[5] N.M.Noor,J.C.Than, O.M.Rijal, R.M.Kassim,A.Yunus, A.A.Zeki, M.Anzidei, L.Saba,and J.S.Suri,“Automatic lung segmentation using control feedback system: morphology and texture paradigm,”J. Med. Syst.,vol. 39, no.3, 22, 2015.

[6] A.R.Pulagam,G.B.Kande, V.K.R.Ede,andR.B.Inampudi,“Automated lung segmentation from HRCT scans with diffuse parenchymal lung diseases,”J. Digit. Imaging, vol. 29, no.4,pp. 507-519, 2016.

[7] Awais Mansoor, Ulas Bagci, Brent Foster, Ziyue Xu, Georgios Z. Papadakis, Les R. Folio, Jayaram K. Udupa, Daniel J. Mollura, "Segmentation and Image Analysis of Abnormal Lungs at CT: Current Approaches, Challenges, and Future Trends" Radiographics ,DOI:10.1148/rg.2015140232

[8] S.H.Chae,H.M.Moon,Y.Chung, J.Shin, and S.B.Pan,"Automatic lung segmentation for large-scale medical image management,”Multimed. Tools Appl., vol.75,no.23,pp. 15347-15363, 2016.

[9] Yamashita, R., Nishio, M., Do, R.K.G. et al. Convolutional neural networks: an overview and application in radiology. Insights Imaging 9, 611-629 (2018). https://doi.org/10.1007/s13244-018o639-

[10] Dou, Q., Chen, H., Yu, L., Qin, J., Heng, P.A., 2016. Multi-level contextual 3D CNNs for false positive reduction in pulmonary nodule detection. IEEE Trans. Biomed. Eng. doi: $10.1109 /$ tbme.2016.2613502

[11] Setio, A .A .A ., Ciompi, F., Litjens, G., Gerke, P., Jacobs, C., van Riel, S., Wille, M.W., Naqibullah, M., Sanchez, C., van Ginneken, B., 2016. Pulmonary nodule detection in CT images: false positive reduction using multi-view convolutional networks. IEEE Trans. Med. Imag. doi: 10.1109/TMI.2016.2536809.

[12] Tongxue Zhou et.al, A review: Deep learning for medical image segmentation using multimodality fusion, Array 3-4 (2019), https://doi.org/10.1016/j.array.2019.100004

[13] Aerts, H. J. W. L., Wee, L., Rios Velazquez, E., Leijenaar, R. T. H., Parmar, C., Grossmann, P., ... Lambin, P. (2019). Data From NSCLC-Radiomics [Data set]. The Cancer Imaging Archive. https://doi.org/10.7937/K9/TCIA.2015.PFoM9REI 
[14] Bakr, Shaimaa; Gevaert, Olivier; Echegaray, Sebastian; Ayers, Kelsey; Zhou, Mu; Shafiq, Majid; Zheng, Hong; Zhang, Weiruo; Leung, Ann; Kadoch, Michael; Shrager, Joseph; Quon, Andrew; Rubin, Daniel; Plevritis, Sylvia; Napel, Sandy.(2017). Data for NSCLC Radiogenomics Collection. The Cancer Imaging Archive. http://doi.org/10.7937/K9/TCIA.2017.7hs46erv

[15] Ronneberger O., Fischer P., Brox T. (2015) U-Net: Convolutional Networks for Biomedical Image Segmentation. In: Navab N., Hornegger J., Wells W., Frangi A. (eds) Medical Image Computing and Computer-Assisted Intervention - MICCAI 2015. MICCAI 2015. Lecture Notes in Computer Science, vol 9351. Springer, Cham

[16] Xu, B., Wang, N., Chen, T. and Li, M., 2015. Empirical evaluation of rectified activations in convolutional network. arXiv preprint arXiv:1505.00853.

[17] Goodfellow, I., Bengio, Y., Courville, A. and Bengio, Y., 2016. Deep learning (Vol. 1). Cambridge: MIT press.

[18] Sebastian Ruder 2016. An overview of gradient descent optimization algorithms. arXiv preprint arXiv:1609.04747

[19] Havaei M., Guizard N., Chapados N., Bengio Y. International Conference on Medical Image Computing and Computer-Assisted Intervention. Springer; 2016. HeMIS: Hetero-modal image segmentation; pp. 469-477.

[20] Selvaraju, R. R., Cogswell, M., Das, A., Vedantam, R., Parikh, D., \& Batra, D. (2016). GradCAM: Visual Explanations from Deep Networks via Gradient-based Localization. arXiv:1610.02391

[21 Wei Y, Shen G, Juan-juan L. A Fully Automatic Method for Lung Parenchyma Segmentation and Repairing, Springer. J Digit Imaging. 2013;26:483-495

[22] Shuangfeng Dai, Ke Lu, Jiyang Dong, Yifei Zhang, and Yong Chen. 2015. A novel approach of lung segmentation on chest CT images using graph cuts. Neurocomput. 168, C (November 2015), 799-807. DOI:https://doi.org/10.1016/j.neucom.2015.05.044

[23] Noor NM, Than JC, Rijal OM, et al. Automatic lung segmentation using control feedback system: morphology and texture paradigm. J Med Syst. 2015;39(3):22. doi:10.1007/s10916-0150214-6

[24 ] Zhang W, Wang X, Zhang P, Chen J. Global optimal hybrid geometric active contour for automated lung segmentation on CT images. Comput Biol Med. 2017;91:168-180. doi:10.1016/j.compbiomed.2017.10.005

[25] H. Koyuncu, "Lupsix: A Cascade Framework for Lung Parenchyma Segmentation in Axial CT Images”, IJISAE, vol. 6, no. 4, pp. 322-328, Dec. 2018. 


\section{Chapter 4}

\section{Auto Delineation of Gross Tumor Volume in NSCLC}

Ravindra Patil, Leonard Wee and Andre Dekker

Proceedings of Machine Learning Research part of arXiv ${ }^{\circledast}$

Under consideration for publication with Springer in Communications in Computer and Information Science (CCIS). 


\begin{abstract}
Lung Cancer is a leading cause of death among different cancers. Identification of the tumor volume is one of the vital steps in diagnosis as well as treatment planning. Ideally, the clinician identifies the region of Gross Tumor Volume (GTV) and delineates manually the region of GTV for treatment planning. This approach involves considerable effort, in terms of time as each slice of the volume needs to be manually contoured and there is variability that exists with respect to different clinicians. In this work, we present a deep learning based automated technique that overcomes various shortcomings of traditional GTV delineation and we were able to achieve a 0.85 dice score in segmentation of Gross Tumor Volume (GTV) for specifically Non-Small Cell Lung Cancer subjects.
\end{abstract}

Keywords: Deep Learning, NSCLC, Gross Tumor Volume (GTV) 


\section{Introduction}

Lung Cancer (LC) is by far the leading cause of death by any cancer. The American Cancer Society estimates the number of cancer deaths in US in 2019 to be 142,670. Being the second most common cancer in both genders, the chances of developing it is 1:15 and 1:17 for men and women respectively. With 228,150 new cases of lung cancer expected to be diagnosed in 2019 in US [1] there is a great need for automating LC diagnosis for screening and treatment planning. Non-small cell lung cancer (NSCLC) is the most common type of LC [2] and radiation therapy (RT) is one of the predominant approach used to treat NSCLC. However, this approach relies on accurate delineation of the Gross Tumor Volume (GTV) on a CT scan which is a manual task performed by clinicians. The approach of manual contouring is time consuming and ranges from 20 minutes to 4 hours per subject of NSCLC based on the disease condition and the institution requirements [3]. In addition, manual contouring is dependent on the experience of the clinician and there is inter clinician variability for the same subject [3]. If auto segmentation is possible, it would aid in significant reduction in the time for contouring and would help in eliminating the inter-observer variability.

Multiple studies have tried to address auto segmentation in the cancer domain using traditional machine learning approaches. Markov random fields were used for target delineation in head and neck radiotherapy [4]. Other studies used active contour $[5]$ and decision tree [6] and $\mathrm{KNN}[7,8]$ approaches in contouring the region of GTV in head and neck cancer. Further, in NSCLC multiple approaches have been used to auto segment the tumor region using atlas-based approaches, active contour, as well mixture based models [9-12]. However, with the advent of Deep Learning techniques, which has the capability to self-learn the pattern rather than requiring hand engineered features, has shown tremendous potential in cancer care [13]. In addition, deep learning techniques have better generalization capabilities and are better suited to handle subtle variation in the subject size, scanner settings and protocol differences compare to the traditional machine learning approaches [14]. 
The objective of this study is to auto segment GTV in CT images of NSCLC subjects using deep learning model with different variations in the histology, scanner settings and the slice thickness and compare that with the existing approaches.

\section{Methodology}

\section{Data Set}

The dataset used for model training of Lung GTV segmentation was obtained from TCIA repository of 422 NSCLC patients [15] (www.cancerimagingarchive.net). This dataset contained pretreatment CT scans where in the GTV regions were manually delineated by a radiation oncologist as a $3 \mathrm{D}$ volume. In total 422 subjects were used for training, which maps to $\sim 42,019$ Lung CT slices on which the algorithm was trained. Out of 422 subjects, randomly 337 subjects were used for model training and remaining 85 for model validation. The split of data can be seen in the infographic of Figure 1. The training data contained the following sub categories of NSCLC: Adenocarcinoma, Large cell carcinoma and Squamous cell carcinoma. The CT scanners used for imaging the subjects were from different vendors (Siemens, CNS Inc, Philips and GE) and the slice thickness varied from 0.65 to $5 \mathrm{~mm}$ with $512{ }^{*} 512$ resolution.

For independent testing the NSCLC-Radiomics-Interobserver [16] dataset, containing 21 subjects of NSCLC was used. These pre-treatment CT scans, had blinded manual delineations by five different radiation oncologists of the $3 \mathrm{D}$ volume of the gross tumor volume on $\mathrm{CT}$. 

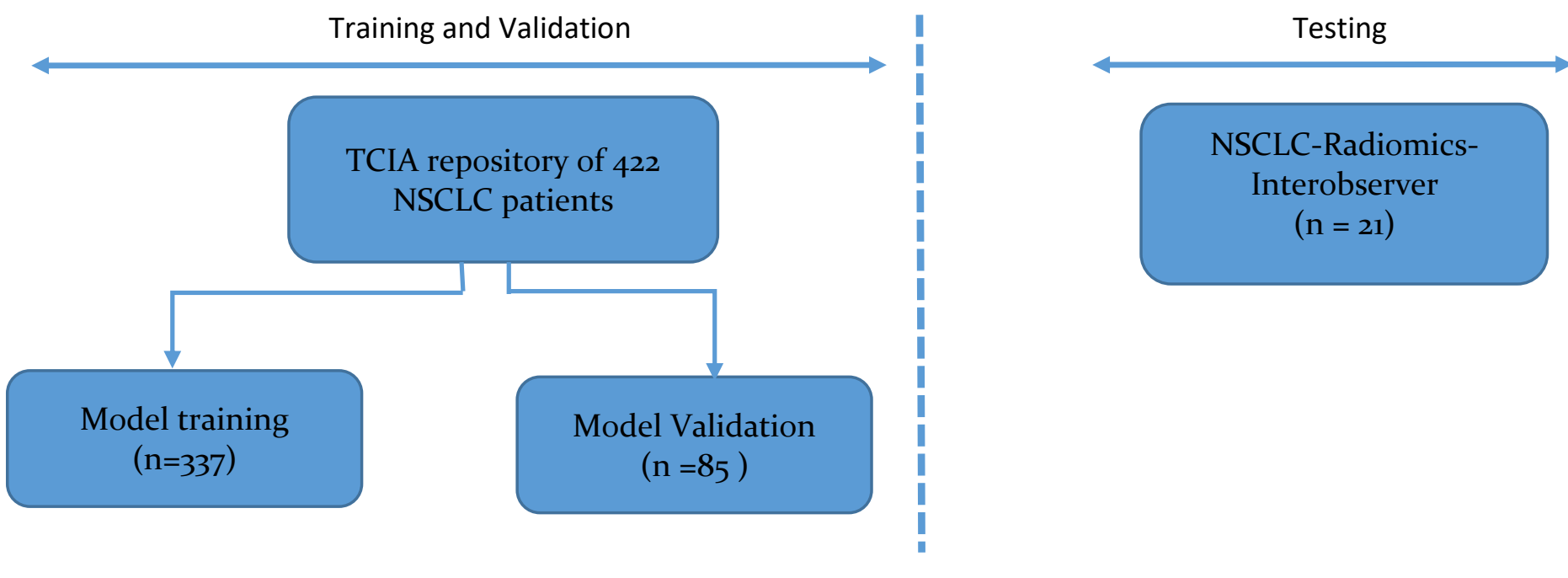

Figure 1. Data Distribution

Our approach of GTV segmentation is described in this section and the overall pipeline is shown in Figure 2. The NSCLC subject's CT volumes were considered and processed in $2 \mathrm{D}$ with each slice as input.

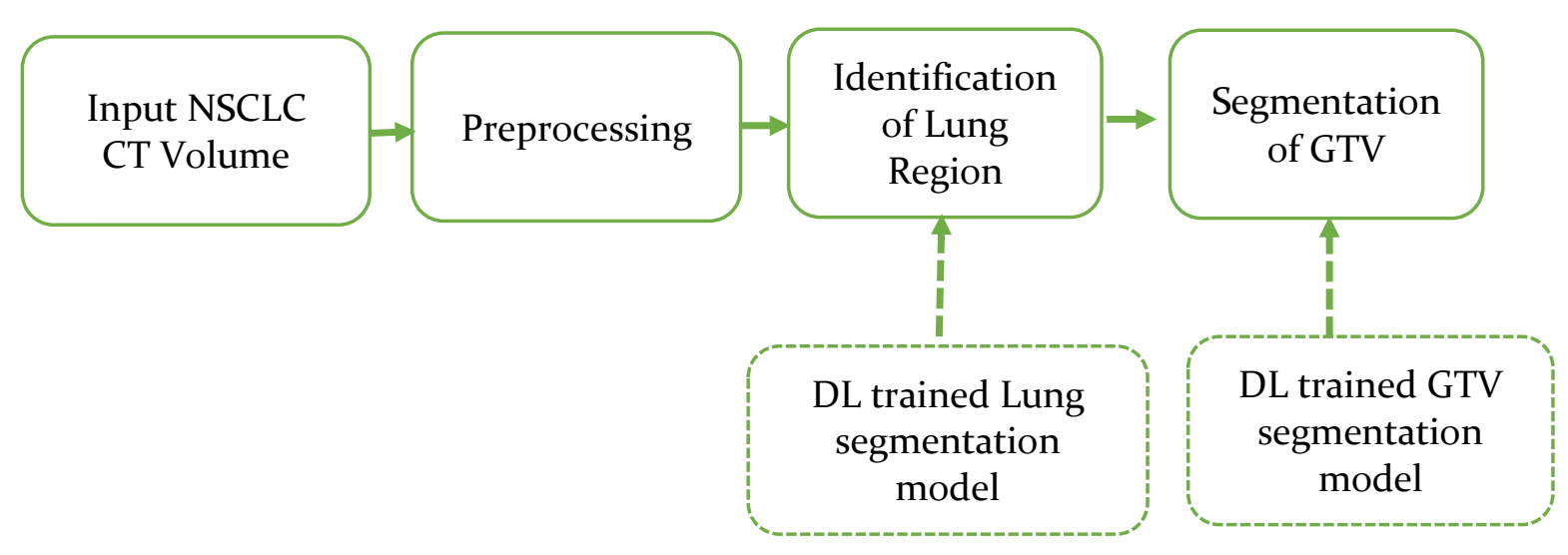

Figure 2. Process flow of GTV segmentation 


\section{Pre processing}

The input CT volumes are processed to set non-anatomical regions such as air (with HU value below -1000) to $\mathrm{o}$, so that the number of the pixels in the computations are reduced. Data augmentation was performed in terms of translation and rotation (o to 30 degrees) to make the model robust against data variations. No specific preprocessing steps such as de-noising, artifact corrections, system-based calibration were employed. The preprocessing approach was specifically designed to have minimum steps, to test the efficacy of convolution neural networks.

\section{Identification of the Lung Region}

In order to reduce the computation effort and to find the relevant scans which has lung region, the DL based lung segmentation module described in chapter 3, is used for lung slice identification. Only slices with a lung region were considered for further model building as well as during the scoring approach. This approach helps in reducing the false positive detection of GTV in non-lung regions, when a full body scan is passed through the model.

\section{Model Training}

The main objective is to segment the GTV given a CT slice of a lung . We employ a modified U-Net [17] inspired CNN architecture to arrive at the GTV segmentation. The initialization of the weights for this network was performed based on a transfer learning approach, wherein the weights of the model of the lung segmentation model (described in chapter 3) are used as initial weights for the GTV model.

We employ a pixel based classification mechanism that aims at classifying whether a pixel belongs GTV region. The model architecture that is built was inspired from U-Net Convolution Neural Network (CNN), which consists of 18 convolutional layers, 4 central pooling layers with the convolutional kernel size of ${ }_{3} \mathrm{X}_{3}$ in each convolutional layer. The schematic representation of the model architecture is as shown in the Figure 3. 


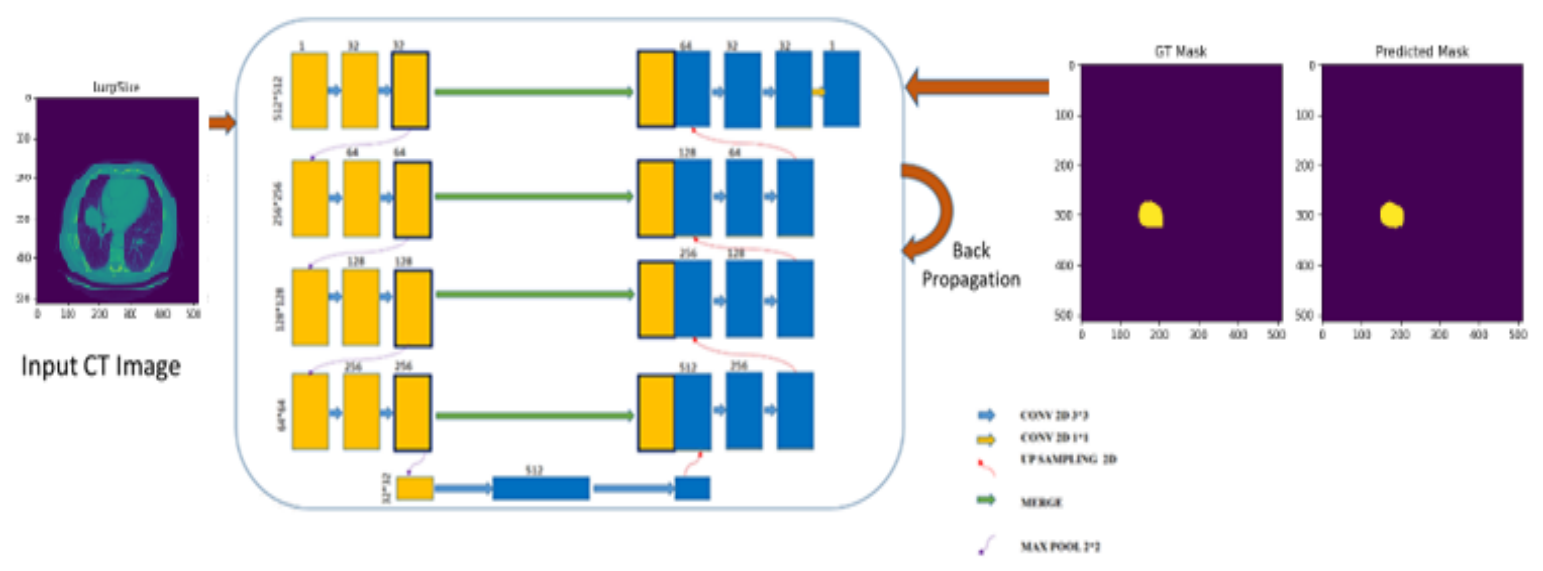

Figure 1: GTV Model Architecture

The convolutional layers perform convolutional operation on all input feature maps to obtain output features defined by the Rectified Linear Units (ReLU) [18] activation function [16]. The feature map layer combination is defined by the equation $(1)$

$$
f^{j}=\operatorname{ReLU}\left(\sum_{i=1}^{m} C^{i j} * f^{i}+b^{j}\right.
$$

Where $f^{i}$ and $f^{j}$ are the ith input feature map and jth output feature map, respectively. We define $C^{i j}$ as the convolutional kernel between $f^{i}$ and $f^{j}\left({ }^{*}\right.$ denotes the 2-D convolutional operation), bj is the bias of the jth output feature map.

After each convolutional layer, a rectified linear unit (ReLU) is used as a non-linear activation function, this is added to bring non linearity to the model and is expressed as:

$$
\operatorname{ReLU}(z)=\max (0, z)
$$

Further to the last convolutional layer, a fully connected layer is applied where each output unit connects to all inputs. This layer can capture correlations between different features produced by the convolutional layer. For achieving non-linearity and a two-class classifier, the sigmoid function was used. Since the sigmoid function ranges from zero to one, it can be directly related to class probabilities making it an ideal activation function for this classification task.

$$
\operatorname{sigmoid}(z)=\frac{1}{1+e^{-z}}
$$


The goal of network training is to maximize the probability of the correct class. This is achieved by minimizing the dice coefficient loss function. The loss function is minimized during the model's training process. The weight update was performed using an Adaptive Moment Estimation (ADAM) algorithm [19]. Instead of adapting the parameter learning rates based on the average first moment as in RMSProp, ADAM makes use of the average of second moments of the gradients. Specifically, the algorithm calculates an exponential moving average of the gradient and the squared gradient, and the parameters $\beta_{1}, \beta_{2}$ control the decay rates of these moving averages. The initial value of the moving averages and $\beta_{1}, \beta_{2}$ values close to 1.0 (recommended) results in a bias of moment estimates towards zero. This bias is overcome by calculating the biased estimates and then calculating bias-corrected estimates. ADAM is an extension to stochastic gradient descent and converges faster than other stochastic optimization methods [19]. It also rectifies problem such as vanishing learning rate, slow convergence that other optimization problems face which leads to fluctuating loss function. The weights are updated based on the below equation

$$
w^{(t+1)} \leftarrow w^{(t)}-\eta \frac{\widehat{m_{w}}}{\sqrt{\widehat{v_{w}}}+\varepsilon}
$$

Where $\varepsilon$ is a small number used to prevent division by zero.

And

$$
\begin{gathered}
\widehat{m_{w}}=\frac{m_{w}^{(t+1)}}{1-\beta_{1}^{t}} \\
\widehat{v_{w}}=\frac{v_{w}^{(t+1)}}{1-\beta_{2}^{t}}
\end{gathered}
$$

Where $m_{w}$ and $v_{w}$ are estimates of the first moment and second moment of the gradients respectively. $\beta_{1}$ and $\beta_{2}$ are the forgetting factors for gradients and second moments of gradients, respectively.

The hyperparameters used for the model training are Optimizer = ADAM, Learning Rate=1.oe-6, Metric $=$ Dice score, Number of Epochs $=50$, Batch Size $=\mathbf{2}$. 


\section{Results and Discussion}

The algorithm was built using a training dataset of 337, a validation dataset of 85 subjects and a testing dataset of 21 CT Subjects. For analyzing the performance of the algorithm, dice similarity coefficient (Dice Score) is used as the primary evaluation criteria to assess the automatic segmentation accuracy; also, this is used as a loss function for the backward propagation in the proposed model. The DSC expressed as in equation (7) provides the amount of overlap between two segmentation results [20], wherein $G_{t}$ is the ground truth segmentation and Auto is the automated segmentation performed by the trained model.

$$
\text { Dice Score }=\frac{2 * V\left(G_{t} \cap \text { Auto }\right)}{V\left(G_{t}\right)+V(\text { Auto })}
$$

In the implementation, RT STRUCT of the delineated GTV on each slice by the radiologist is considered as the ground truth and the region predicted by the model is overlaid on the RTSTRUCT mask to arrive at the DSC. The sample output of the algorithm delineating GTV over different variations of size and the location is shown in the figure 4. In the fig 4 the first image in a row indicates the input CT lung slice, the middle image is the contouring performed by the clinician and the last image in a row is predicted contour of GTV by the algorithm. It can be observed that the algorithm was able to detect the GTV, irrespective of position in the lung region and with different variation in size and the texture. 

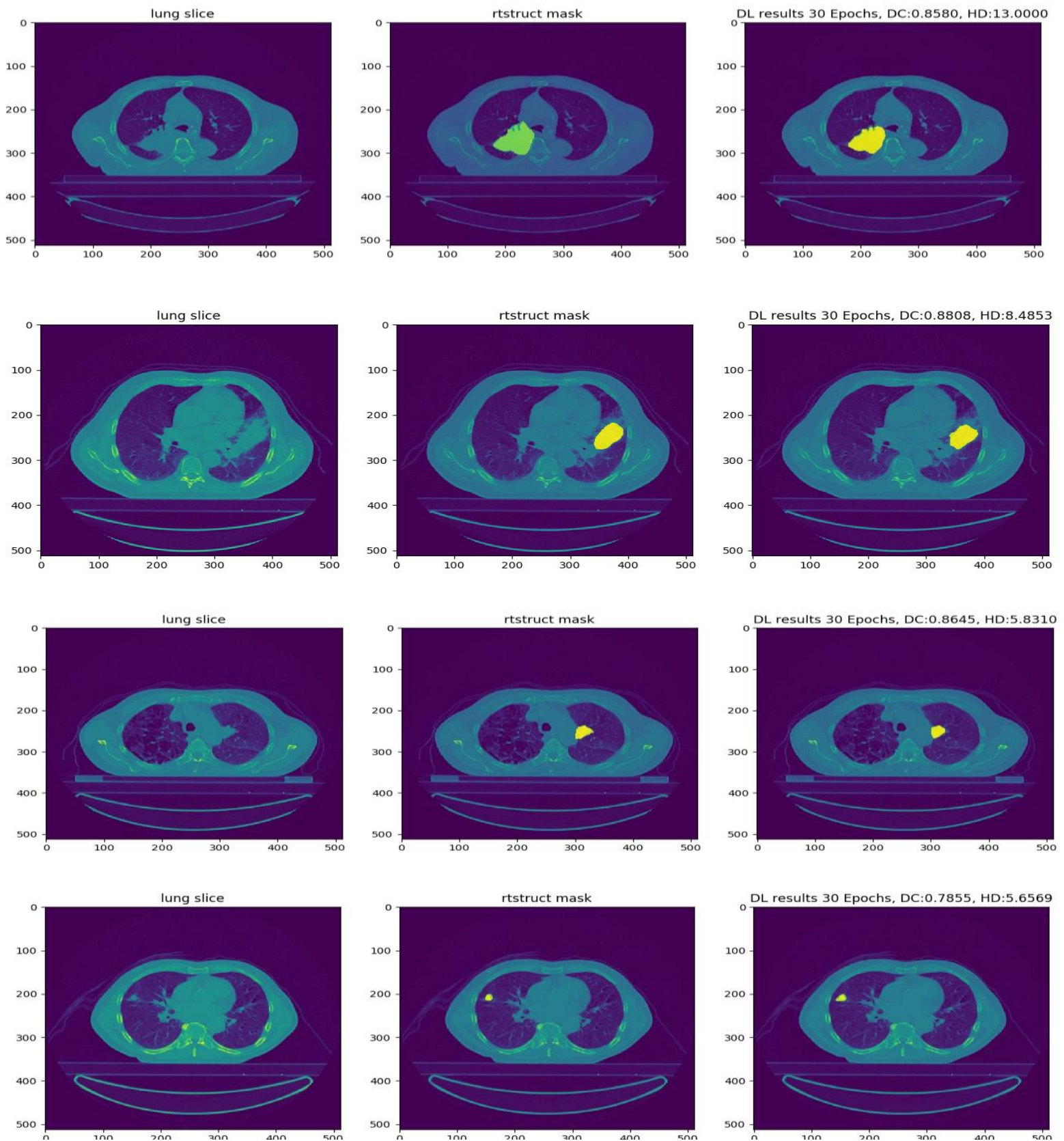

Figure 4. Segmented GTV by the algorithm

Further, the objective analysis was performed based on the dice score on the validation data $(n=85)$ to assesses the role of size of tumor. It can be observed from the figure 5 that the larger the size of the tumor region, the better is the accuracy of the model's GTV region. 


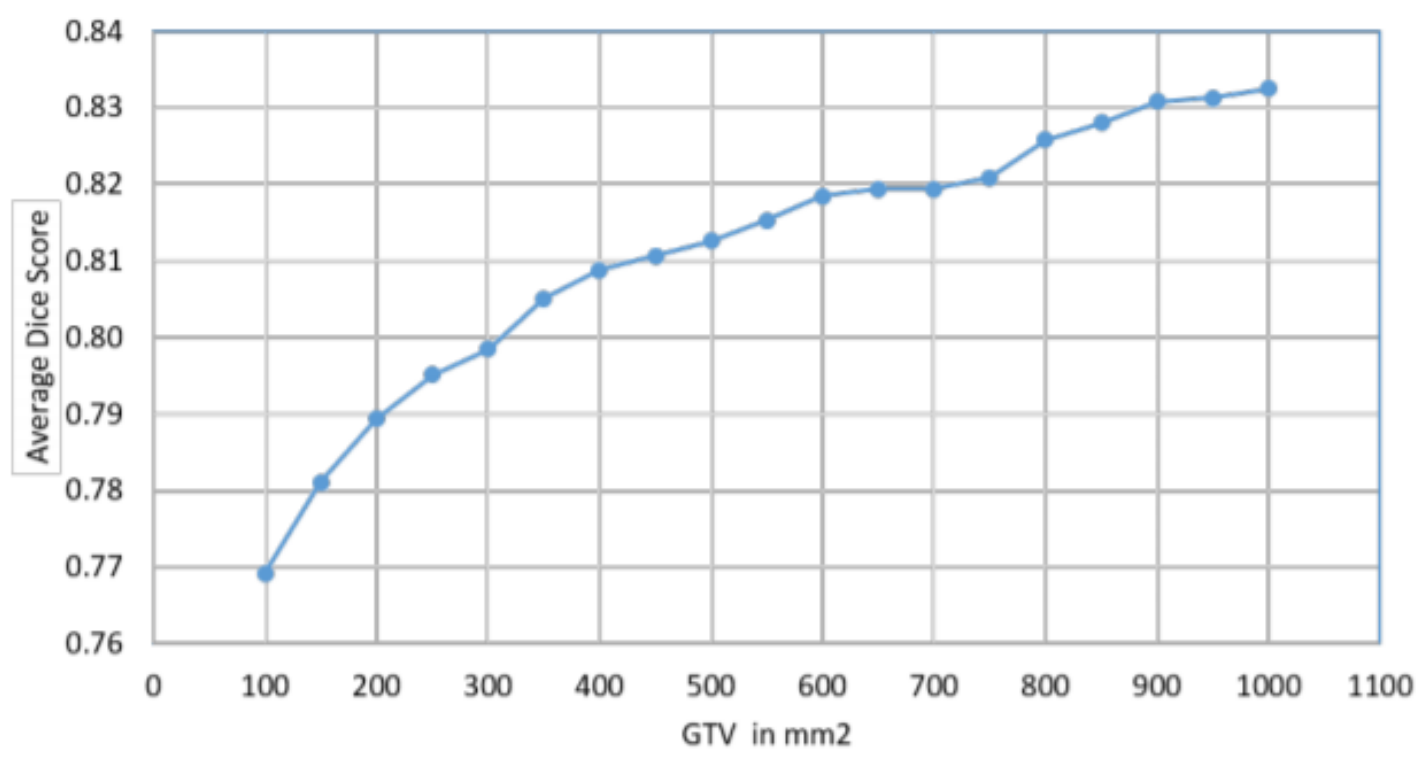

Figure 5. Variation of detection Dice Score to the size of the GTV

In order to bench mark the model on the unseen data, independent data set of 21 subjects were used to test the model efficacy. A mean accuracy of 0.73 DSC was observed with median being 0.74 and maximum dice score being 0.88 on the test data.

It was also observed that the model is biased towards the larger tumor volume, in one of the subject as seen in Figure 6, the algorithm was able to detect the larger tumor size however, and it missed to identify the smaller nodule present in the same slice.
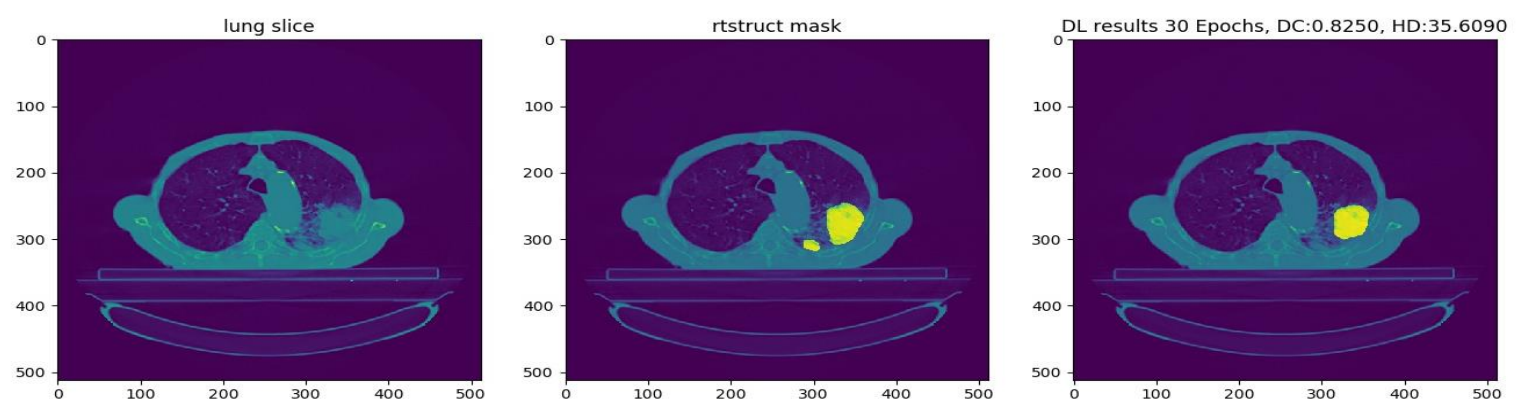

Figure 6. Missed smaller nodule

Further, analysis suggested the train data had larger tumor sizes compared to the smaller nodules leading to this bias in the model. However, the model was able to detect the GTV on CT slices with varied scanner settings and the slice thickness. 
The GTV segmentation was performed using NVIDIA GTX 1080 GPU in 15 seconds per scan on average; this is significant improvement from manual delineation that generally takes 20 minutes -4 hours per scan. This automated approach without manual involvement of radiologist leads to saving in diagnosis/treatment time.

\section{Conclusion}

The results show the robust nature of the DL algorithm to segment the tumor regions in the NSCLC subjects. This approach demonstrate the automation in the segmentation process, without using any seed point input. The same algorithm might also be extended to other anatomical structures such as Liver, Head and Neck. This would further aid in improving the efficiency in the workflow of the cancer care. 


\section{References}

[1] https://www.cancer.org/cancer/lung-cancer/about/key-statistics.html, last accessed 25-02-2020.

[2] Zappa C, Mousa SA. Non-small cell lung cancer: current treatment and future advances. Transl Lung Cancer Res. 2016 Jun;5(3):288-300. doi: 10.21037/tlcr.2016.o6.o7. PMID: 27413711; PMCID: PMC4931124.

[3] Bi N, Wang J, Zhang T, Chen X, Xia W, Miao J, Xu K, Wu L, Fan Q, Wang L, Li Y, Zhou Z, Dai J. Deep Learning Improved Clinical Target Volume Contouring Quality and Efficiency for Postoperative Radiation Therapy in Non-small Cell Lung Cancer. Front Oncol. 2019 Nov 13;9:1192. doi: 10.3389/fonc.2019.01192. PMID: 31799181; PMCID: PMC6863957.

[4] Yang J, Beadle BM, Garden AS, Schwartz DL, Aristophanous M, A multimodality segmentation framework for automatic target delineation in head and neck radiotherapy, Med Phys. 2015 Sep; 42(9):5310-20

[5] Zeng Z, Wang J, Tiddeman B, Zwiggelaar R, Unsupervised tumour segmentation in PET using local and global intensity-fitting active surface and alpha matting. Comput Biol Med. 2013 Oct; 43(10):1530-44.

[6] Berthon B, Evans M, Marshall C, Palaniappan N, Cole N, Jayaprakasam V, Rackley T, Spezi E, Head and neck target delineation using a novel PET automatic segmentation algorithm. Radiother Oncol. 2017 Feb; 122(2):242-247.

[7] Coregistered FDG PET/CT-based textural characterization of head and neck cancer for radiation treatment planning.Yu H, Caldwell C, Mah K, Mozeg D IEEE Trans Med Imaging. 2009 Mar; 28(3):374-83.

[8] Comelli A., Stefano A., Benfante V., Russo G. Normal and abnormal tissue classification in positron emission tomography oncological studies. Pattern Recognition and Image Analysis. 2018;28(1):106-113. doi: 10.1134/s1054661818010054

[9] Lustberg T, van Soest J, Gooding M, Peressutti D, Aljabar P, van der Stoep J, et al. Clinical evaluation of atlas and deep learning based automatic contouring for lung cancer. Radiother Oncol. (2018) 126:312-7. doi: 10.1016/j.radonc.2017.11.012

[10] Comelli A, Stefano A, Bignardi S, Russo G, Sabini MG, Ippolito M, et al. Active contour algorithm with discriminant analysis for delineating tumors in positron emission tomography. Artif Intell Med. (2019) 94:67-78. doi: 10.1016/j.artmed.2019.01.002 
[11] Zhuang M, Dierckx RA, Zaidi H. Generic and robust method for automatic segmentation of PET images using an active contour model. Med Phys. (2016) 43:4483. doi: $10.1118 / 1.4954844$

[12] Giri MG, Cavedon C, Mazzarotto R, Ferdeghini M. A Dirichlet process mixture model for automatic. (18)F-FDG PET image segmentation: validation study on phantoms and on lung and esophageal lesions. Med Phys. (2016) 43:2491. doi: $10.1118 / 1.4947123$

[13] AdrianB, Levine Colin, Schlosser, Jasleen Grewal, Robin Coope, et.al Rise of the Machines: Advances in Deep Learning for Cancer Diagnosis, Trends in Cancer, Volume 5, Issue 3, March 2019, Pages 157-169

[14] Behnam Neyshabur, Srinadh Bhojanapalli, David McAllester, Nathan Srebro, Exploring Generalization in Deep Learning, 31st Conference on Neural Information Processing Systems (NIPS 2017), Long Beach, CA, USA.

[15] Aerts, H. J. W. L., Wee, L., Rios Velazquez, E., Leijenaar, R. T. H., Parmar, C., Grossmann, P., Lambin, P. (2019). Data From NSCLC-Radiomics [Data set]. The Cancer Imaging Archive. https://doi.org/10.7937/K9/TCIA.2015.PFoMgREI

[16] Wee, L., Aerts, H. J.L., Kalendralis, P., \& Dekker, A. (2019). Data from NSCLCRadiomics-Interobserver. The Cancer Imaging Archive. https://doi.org/10.7937/tcia.2019.cwvlpd26.

[17] Ronneberger O., Fischer P., Brox T. (2015) U-Net: Convolutional Networks for Biomedical Image Segmentation. In: Navab N., Hornegger J., Wells W., Frangi A. (eds) Medical Image Computing and Computer-Assisted Intervention - MICCAI 2015. MICCAI 2015. Lecture Notes in Computer Science, vol 9351. Springer, Cham

[18] Xu, B., Wang, N., Chen, T. and Li, M., 2015. Empirical evaluation of rectified activations in convolutional network. arXiv preprint arXiv:1505.00853.

[19] Goodfellow, I., Bengio, Y., Courville, A. and Bengio, Y., 2016. Deep learning (Vol. 1). Cambridge: MIT press.

[20] Havaei M., Guizard N., Chapados N., Bengio Y. International Conference on Medical Image Computing and Computer-Assisted Intervention. Springer; 2016. HeMIS: Hetero-modal image segmentation; pp. 469-477. 


\section{Chapter 5}

\section{Does Radiomics Improves Survival Prediction of NSCLC?}

Ravindra Patil, Geetha M, Andre Dekker

Journal of Thoracic Oncology, November 2017 Volume 12, Issue 11, Supplement 2, Page S2089

DOI: https://doi.org/10.1016/j.jtho.2017.09.1154. 


\begin{abstract}
Non-small cell lung cancer (NSCLC) accounts for $85 \%$ of all the lung cancers worldwide. An accurate survival time prediction is important so that the patient can plan her/his activities and it might aid physicians in recommending the best care plan. There have been multiple studies that reported building survival analysis models for lung cancer. The factors that are considered in most of these models include age, gender, tumor size, weight loss, smoking history and TNM staging to arrive at the survival prediction. However, the current focus is to make the prediction of the survival analysis more personalized and accurate using Radiomics. This work highlights different models that were built by considering clinical features, Radiomics features and combination of Radiomics and clinical features to predict the survival analysis. Further, concordance index of 0.69 was achieved when the clinical and Radiomics features were combined to predict the survival analysis. In addition, separate models were built based on the histology of NSCLC to understand the role of histology in survival prediction.
\end{abstract}

Keywords : Survival Model, Lung Cancer, NSCLC 


\section{Introduction}

Non small cell lung cancer accounts for $85 \%$ of all lung cancers and also significant decision making process is required in each stage of the disease [1]. In practice, NSCLC is characterized into one of the following sub categories viz. Large cell carcinoma, Adenocarcinoma and Squamous cell carcinoma. In most of the cases, these carcinomas are diagnosed at advanced stages leading to poor prognosis [2]. Early stage detection of NSCLC leads to better prognosis as the ability to modify the outcome is then quite high. In current best practice, approximately $20-25 \%$ of the cases are detected at the early stage ((IA-IIB) [3, 4]. Surgical intervention is the most followed approach for patients with stage I and II NSCLC [5]. In stage III patients there is no clear consensus on the role of surgery and stage IV are commonly not considered for surgical procedures [6]. The study by Cerfolio et al [7] revealed that about $14 \%$ of the NSCLC patients were clinically over-staged and close to $32 \%$ were under-staged clinically. The correlation between the pathological and clinical staging in stage I NSCLC was observed to be just 56\% [7]. Although, Tumor Node Metastasis (TNM) staging system is considered as the gold standard to arrive at the staging, however there are still discrepancies as staging based on TNM is not personalized, leading to incorrect staging with respect the severity of the disease [8].

An accurate survival time prediction is important so that a patient can plan her/his activities and it might aid physicians to recommend the best care plan [9]. Multiple studies have built survival analysis models for lung cancer [10, 11]. The factors that are considered in most of these models include Age, gender, tumor size, weight loss, smoking history, TNM staging to arrive at the survival prediction [12]. The current focus is to make the prediction of the survival analysis (even) more personalized and accurate [13]. Radiomics, which deals with extraction of quantifiable features from the CT images, promises to aid in personalized medicine [14]. In this work, we validate the use of Radiomic features and arrive at a Radiomic signature, which is hypothesized to have better prediction power than clinical parameters. In addition, we analyze the role of radiomic features in improving the accuracy of survival prediction in NSCLC. Further, 
the role of histology was ascertained by building individual models for each of the histology's, considering Radiomic signature.

\section{METHODOLOGY}

The pipeline of processes adapted in this work is depicted in Figure 1. It involves CT image acquisition, segmentation, feature selection, feature extraction and predictive survival analysis using a Cox's proportional hazard model.

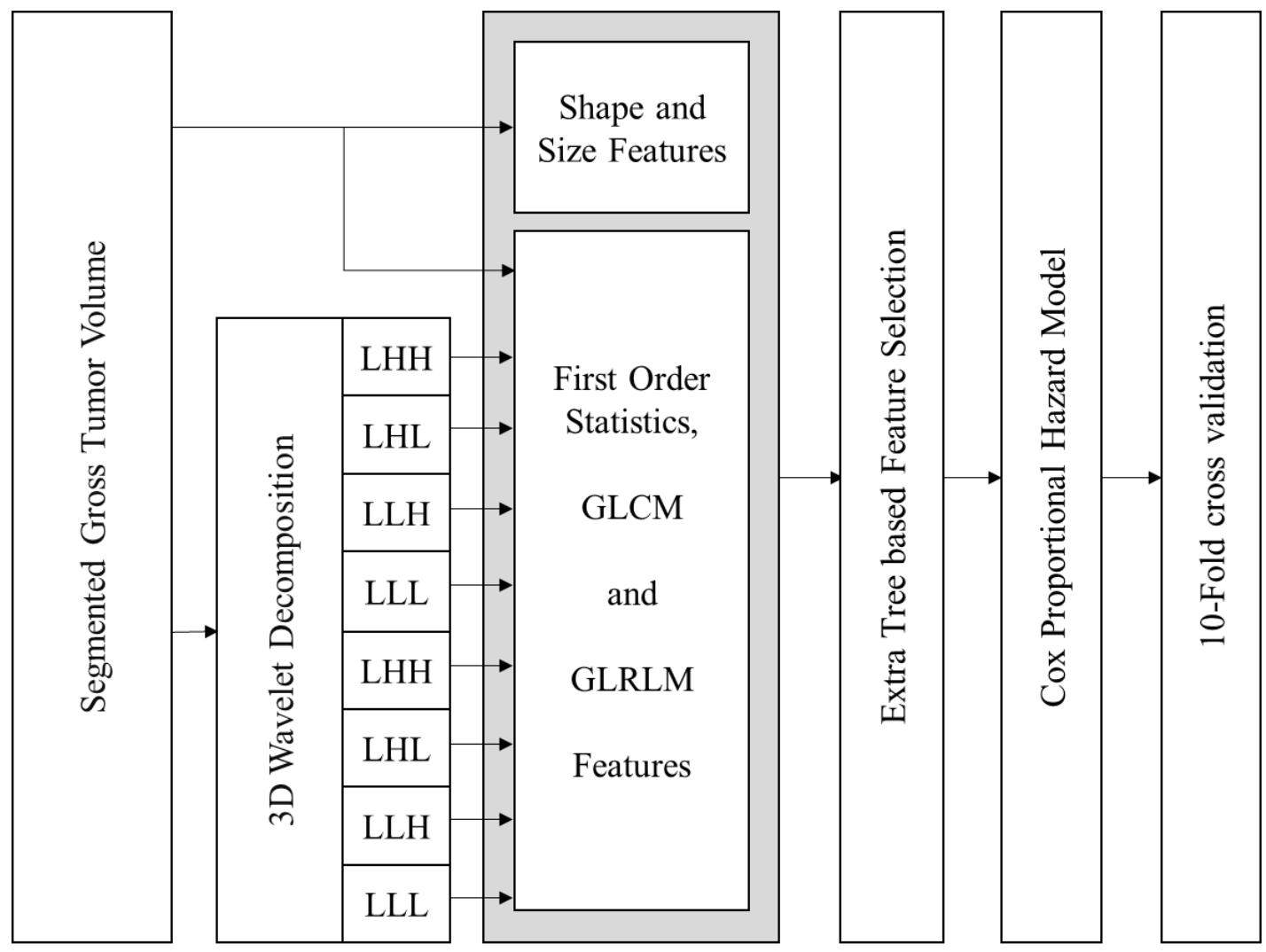

Figure 1. Proposed Methodology and Workflow

The approach followed in this work involves extraction of radiomic features from segmented gross tumor volume of the CT images. The significant features are selected based on feature selection approach from the exhaustive list of radiomic features $\mathrm{s}$ and a cox proportional hazard analysis is performed to predict the survival of the subjects with different histology's of NSCLC. 


\section{Data}

The NSCLC-Radiomics dataset (http://www.cancerimagingarchive.net) [15] containing CT data in DICOM format has been used for this study. The subjects belonging to three different categories of histology i.e., adenocarcinoma, large cell carcinoma and squamous cell carcinoma have been considered. The demographic distribution of the subjects of the data set mentioned in Table 1. In addition, the clinical information associated with each of the subject is captured which includes age, gender, smoking history, histology, T stage, N Stage and overall TNM stage. The demographic and clinical characteristics of the subjects are provided in Table 1.

\begin{tabular}{|l|l|}
\hline Age $(\mathrm{y})$ & $68.21 \pm 9 \cdot 58$ \\
\hline Gender & \\
Male & $164(69.2 \%)$ \\
Female & $73(30.8 \%)$ \\
\hline Histology & \\
Adenocarcinoma & $39(16.4 \%)$ \\
Large cell & $102(43.0 \%)$ \\
Squamous cell carcinoma & $96(40.5 \%)$ \\
\hline Deadstatus & \\
Alive (o) & $102(43.0 \%)$ \\
Dead (1) & $135(57.0 \%)$ \\
\hline T stage & $37(15.6 \%)$ \\
T1 & $92(38.8 \%)$ \\
T2 & $32(13.5 \%)$ \\
T3 & $76(32.06 \%)$ \\
T4 & \\
\hline N stage & \\
No & $81(34.1 \%)$ \\
N1 & $15(6.3 \%)$ \\
N2 & $83(35.0 \%)$ \\
N3 & $55(23.2 \%)$ \\
N4 & $3(1.3 \%)$ \\
\hline M Stage & \\
Mo & $234(98.7 \%)$ \\
M3 & $3(1.2 \%)$ \\
\hline & \\
\hline
\end{tabular}




\begin{tabular}{|l|l|}
\hline Overall.Stage & \\
I & $37(15.6 \%)$ \\
II & $22(9.28 \%)$ \\
IIIa & $65(27.42 \%)$ \\
IIIb & $113(47.67 \%)$ \\
\hline
\end{tabular}

Table 1. The demographic and clinical characteristics of the subjects

\section{Segmentation}

Segmentation plays a crucial role in performing a reliable analysis on the region of interest. In the dataset adopted for this study, an experienced radiation oncologist performed the segmentation of gross tumor volume (GTV) of the primary tumor. In order to determine the ceiling of the predictor performance, we have used these manually generated masks instead of those which are automatically generated. Subsequently registration was performed on to the CT image with respect to that of the RTSTRUCT provided by the oncologist to extract the GTV. Furthermore, preprocessing and normalization of the data has been avoided to prevent loss of any clinical information that is present.

\section{Feature Extraction}

The radiomic features were extracted from the delineated region of GTV and a total of 431 radiomic features were computed and were divided into Shape and Size features, Texture (GLCM and GLRLM), wavelets and First order statistics. All the features were extracted using custom-built software in MATLAB 2014a. The features belonging to each category are tabulated in Table 2. These features have been used in medical image discrimination and a detailed description can be found in [14]. 


\begin{tabular}{|c|c|c|c|}
\hline Shape and Size & First Order Statistics & GLCM & GLRLM \\
\hline Compactness 1 & Energy & Autocorrelation & SRE \\
\hline Compactness 2: & Entropy & $\begin{array}{l}\text { Cluster } \\
\text { Prominence }\end{array}$ & LRE \\
\hline $\begin{array}{l}\text { Maximum } \\
\text { diameter }\end{array}$ & Kurtosis & Cluster Shade & GLN \\
\hline Spherical & Maximum & $\begin{array}{l}\text { Cluster } \\
\text { Tendency }\end{array}$ & RLN \\
\hline & & 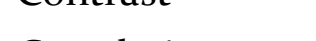 & \\
\hline Surface Area & Mean absolute deviation & Correlation & LGRE \\
\hline $\begin{array}{l}\text { Surface to volume } \\
\text { ratio } \\
\text { Volume }\end{array}$ & $\begin{array}{l}\text { Median } \\
\text { Minimum } \\
\text { Range } \\
\text { RMS value } \\
\text { Skewness } \\
\text { Standard Deviation } \\
\text { Uniformity } \\
\text { Variance }\end{array}$ & $\begin{array}{l}\text { Difference of } \\
\text { Entropy } \\
\text { Dissimilarity } \\
\text { Energy } \\
\text { Entropy } \\
\text { Homogeneity } 1 \\
\text { Homogeneity } 2 \\
\text { Information } \\
\text { measure } \\
\text { correlation } 1 \\
\text { Informational } \\
\text { measure } \\
\text { correlation } 2 \\
\text { Inverse } \\
\text { Difference } \\
\text { Moment } \\
\text { Normalized } \\
\text { Inverse } \\
\text { Difference } \\
\text { Normalized } \\
\text { Inverse variance } \\
\text { Maximum } \\
\text { Probability } \\
\text { Sum average } \\
\text { Sum Entropy } \\
\text { Sum variance } \\
\text { Variance }\end{array}$ & $\begin{array}{l}\text { HGRE } \\
\text { SRLGE } \\
\text { SRHGE } \\
\text { LRLGE } \\
\text { LRHGE }\end{array}$ \\
\hline
\end{tabular}

Table 2: Description of the different categories of features extracted in this work 


\section{Feature Selection}

The feature dimension of the extracted features is almost twice as large as the number of data. This might result in overfitting and would fail to generalize the predictor model. Therefore, a feature selection stage is implemented. In this, an extra tree regressor [16] is trained using the data and the features contributing to the largest Gini importance measure are selected. The Gini importance is described in equation (1)

$$
I_{G}(\theta)=\sum_{T} \sum_{\tau} \Delta i_{\theta}(\tau, T)
$$

Here, $I_{G}$, the genie importance of the feature $\theta$ is calculated by the summation of the Gini impurity loss at each node $\tau$ of each tree T as described in [17].

\section{Model Building}

The Cox Proportional Hazard Model ( $\mathrm{CPH})$ [18] is a form of survival modelling that helps in formulating the survival of the subjects across time. It consists of a baseline part that is dependent on time and an exponential part that is dependent on the covariates (features). The $\mathrm{CPH}$ models the interaction of features and the time taken for occurrence of an event. The Cox survival function denotes the probability of survival of a subject until time $t$ and is formulated as shown in equation (2).

$$
C P H(t, \theta)=h_{0}(t) \exp \left(b_{0}+b_{1} \theta_{1}+b_{2} \theta_{2}+. .\right)
$$

where, $h_{0}(t)$ is the baseline and $b_{0}, b_{1}, .$. are the feature weights fitted on the data using the method of partial-likelihood. The $\mathrm{CPH}$ is considered semi-parametric as $h_{0}(t)$ is not explicitly defined and can take any form. However, once the model is fit, an estimate of the baseline component can be derived. This baseline component can also be separately derived for stratified data and interesting interactions could be studied. In this, each stratum is modelled to have different baseline components and the same parametric coefficients. Therefore, it prevents any dependencies between stratifying covariates and time. 
The cumulative hazard $H(t, \theta)$ is another metric that is used to analyze the prognosis of the histology. It is derived from the Cox survival function as shown in equation (3). It assists in studying interactions within the stratified covariates in finer detail.

$$
H(t, \theta)=-\ln S(t, \theta)
$$

In this study, an analysis is performed on the survival days of subjects diagnosed with three histologies by stratification of the data. The number of days of survival until the event death is modelled using the $\mathrm{CPH}$. The model is created on randomly chosen $60 \%$ of the subjects to eliminate bias due to subject data. Subsequently, the validation of the model is performed using 10 fold cross validation. 


\section{Results and Discussion}

Experiments were performed to determine to the role of clinical parameters of the subjects (such as Age, Gender, TNM stage ), Radiomics features and the combination of clinical parameters and Radiomics features to predict the survival of the subjects for the different histology of NSCLC. The base line Kaplan-Meier (KM) curves considering clinical parameters alone is shown in the figure 2. The concordance index on the validation data was 0.561 .
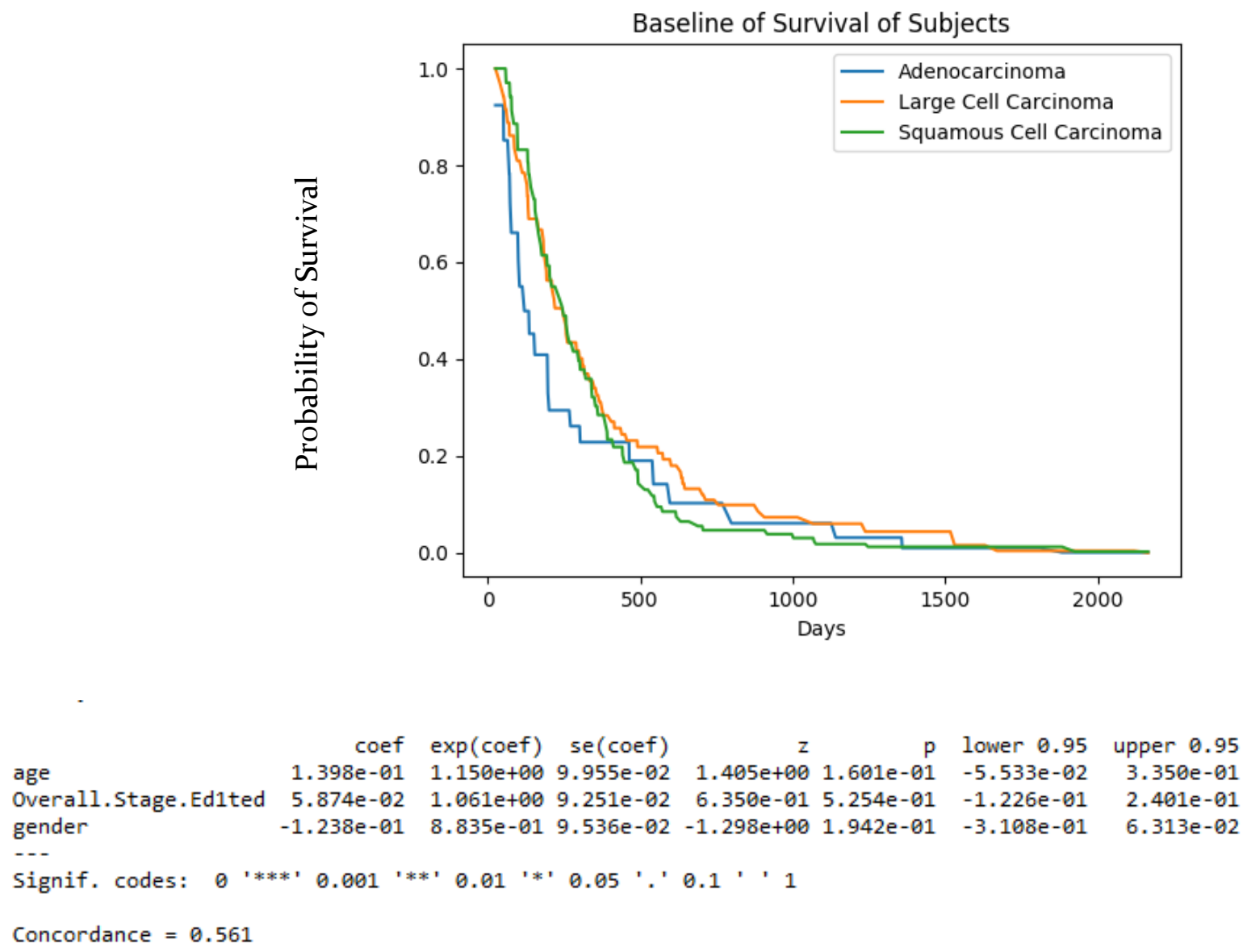

Figure 2. Baseline KM curves of subjects survival considering subject metadata and the associated model features

Another experiment, wherein only the Radiomics features extracted from the GTV were considered, resulted in the concordance index of 0.619 and the baseline curve as seen in Figure 3. 

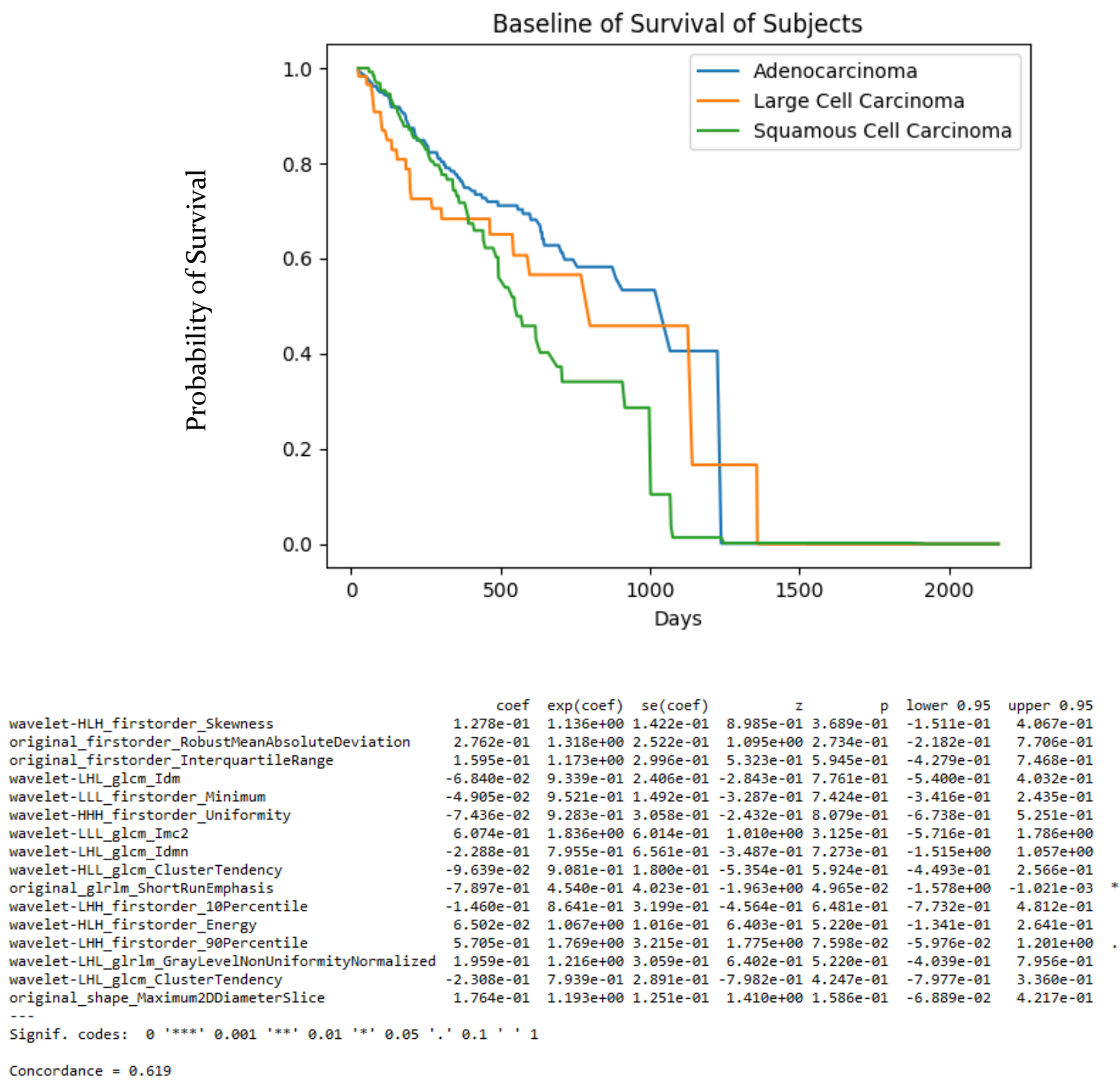

Figure 3. Baseline KM curves of subjects survival considering Radiomics features and the associated model features

The features based on first order, GLRLM and wavelets are significant contributor towards the outcome prediction.

Furthermore, another model was built with the combination of clinical parameters as well as the Radiomic features to ascertain the prognostic value, it 
resulted into a concordance index of 0.69 with significant features being GLRM and GLCM based, wavelets based, age and gender as shown in figure 4 .
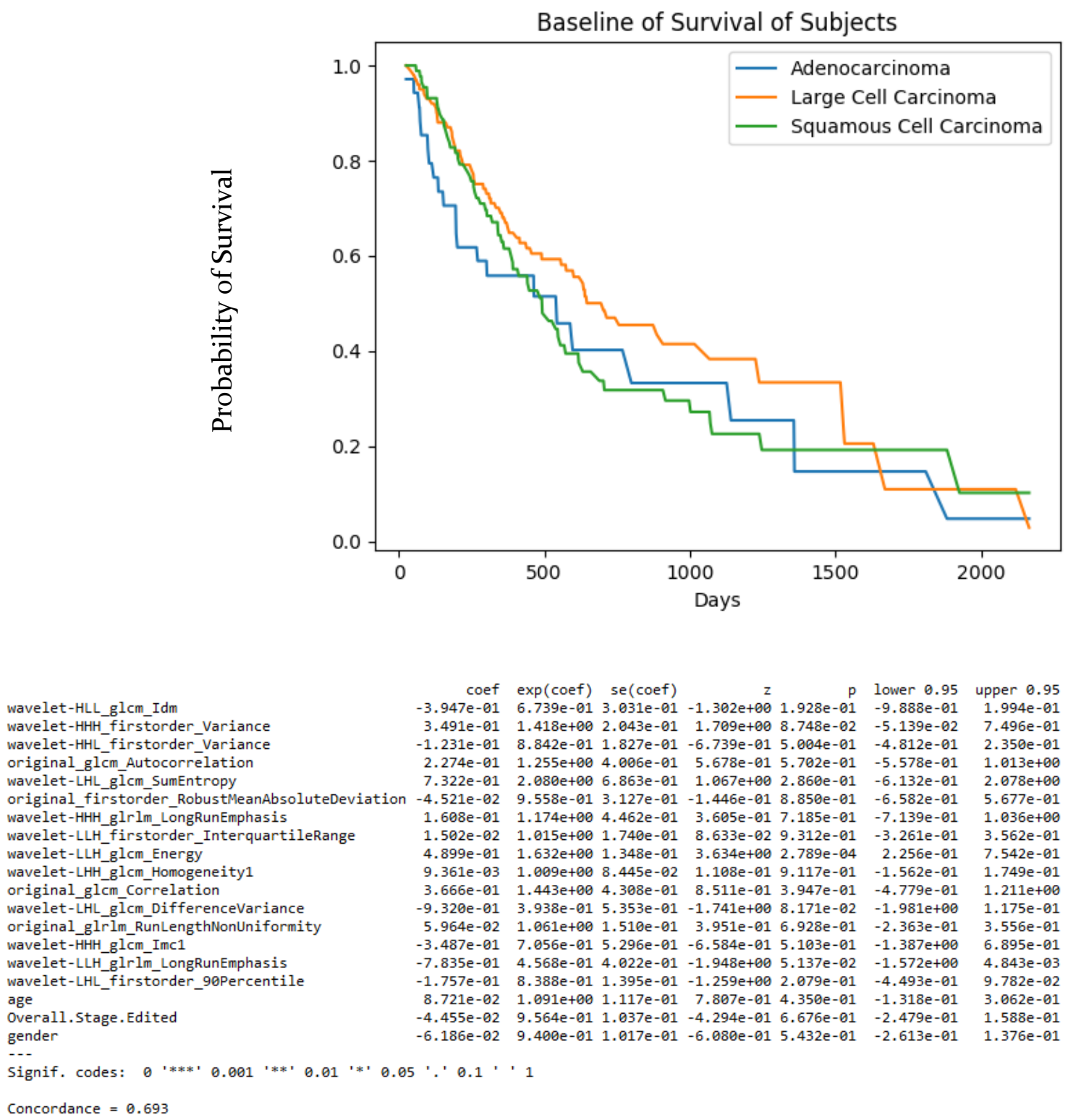

Concordance $=0.693$

Figure 4. Baseline of KM curves subjects survival considering Radiomics and metadata and the associated model features

The hypothesis of Radiomics improving the survival prediction could be observed from the improved concordance index from 0.56 considering only clinical parameters to that of 0.69 when Radiomics features were combined with clinical parameters of the 
subject. These experiments suggests that imaging features could aid in improving the prediction capability of the survival estimation models.

\section{Conclusion}

In this work, it was demonstrated that Radiomics features combined with the clinical parameters helps in improving the survival estimation in NSCLC. Further, in future, integrating this model with auto GTV segmentation would help in automated computation of the survival estimation without manual intervention. In addition, this solution would help in personalized survival prediction leading to better planning in cancer care. 


\section{References}

[1] Molina JR, Yang P, Cassivi SD, Schild SE, Adjei AA. Non-small cell lung cancer: epidemiology, risk factors, treatment, and survivorship. Mayo Clin Proc. 2008 May;83(5):584-94. doi: 10.4065/83.5.584. PMID: 18452692; PMCID: PMC2718421.

[2] Blandin Knight S, Crosbie PA, Balata H, Chudziak J, Hussell T, Dive C. Progress and prospects of early detection in lung cancer. Open Biol. 2017 Sep;7(9):170070. doi: 10.1098/rsob.170070. PMID: 28878044; PMCID: PMC5627048.

[3] Chansky K, Sculier JP, Crowley JJ, et al. The International Association for the Study of Lung Cancer Staging Project: prognostic factors and pathologic TNM stage in surgically managed non-small cell lung cancer. J Thorac Oncol 2009;4(7):792-801.

[4] Scott WJ, Howington J, Feigenberg S, Movsas B, Pisters K; American College of Chest Physicians. Treatment of non-small cell lung cancer stage I and stage II: ACCP evidencebased clinical practice guidelines (2nd edition). Chest. 2007;132(3 Suppl):234S-242S. doi:10.1378/chest.07-1378

[5] NCCN Clinical Practice Guidelines in Oncology. Non-Small Cell Lung Cancer (v.2.2013). 2013. http://www.nccn.org/professionals/physician_gls/pdf/nscl. pdf. Accessed July 2, 2013.

[6] Majem, M., Hernández-Hernández, J., Hernando-Trancho, F. et al. Multidisciplinary consensus statement on the clinical management of patients with stage III non-small cell lung cancer. Clin Transl Oncol 22, 21-36 (2020). https://doi.org/10.1007/s12094-01902134-7

[7] Cerfolio RJ, Bryant AS. Survival of patients with true pathologic stage I non-small cell lung cancer. Ann Thorac Surg. 2009;88:917-922. doi: 10.1016/j.athoracsur.2009.05.040. discussion 922-923.

[8] Zappa C, Mousa SA. Non-small cell lung cancer: current treatment and future advances. Transl Lung Cancer Res. 2016 Jun;5(3):288-300. doi: 10.21037/tlcr.2016.06.o7. PMID: 27413711; PMCID: PMC4931124.

[9] Singh R, Mukhopadhyay K. Survival analysis in clinical trials: Basics and must know areas. Perspect Clin Res. 2011 Oct;2(4):145-8. doi: 10.4103/2229-3485.86872. PMID: 22145125; PMCID: PMC3227332.

[10] Chansky K, Subotic D, Foster NR, Blum T. Survival analyses in lung cancer. J Thorac Dis. 2016 Nov;8(11):3457-3463. doi: 10.21037/jtd.2016.11.28. PMID: 28066627; PMCID: $\mathrm{PMC}_{5179386 .}$

[11] Abedi S, Janbabaei G, Afshari M, et al. Estimating the Survival of Patients With Lung Cancer: What Is the Best Statistical Model?. J Prev Med Public Health. 2019;52(2):140144. doi:10.3961/jpmph.17.090 
[12] Alexander M, Wolfe R, Ball D, Conron M, Stirling RG, Solomon B, MacManus M, Officer A, Karnam S, Burbury K, Evans SM. Lung cancer prognostic index: a risk score to predict overall survival after the diagnosis of non-small-cell lung cancer. $\mathrm{Br} J$ Cancer. 2017 Aug 22;117(5):744-751. doi: 10.1038/bjc.2017.232. Epub 2017 Jul 20. PMID: 28728168; PMCID: PMC5572183.

[13] Yoon J, Zame WR, Banerjee A, Cadeiras M, Alaa AM, et al. (2018) Personalized survival predictions via Trees of Predictors: An application to cardiac transplantation. PLOS ONE 13(3): eo194985. https://doi.org/10.1371/journal.pone.0194985

[14] Hugo J. W. L. Aerts, Emmanuel Rios Velazquez, Ralph T. H. Leijenaar, Chintan Parmar, Patrick Grossmann, Sara Carvalho, Johan Bussink, René Monshouwer, Benjamin Haibe-Kains, Derek Rietveld, Frank Hoebers, Michelle M. Rietbergen, C. René Leemans, Andre Dekker, John Quackenbush, Robert J. Gillies \& Philippe Lambin. Decoding tumour phenotype by noninvasive imaging using a quantitative Radiomics approach. Nature Communications 5, Article number: 4006 (2014) doi:10.1038/ncomms50o6.

[15] Aerts, H. J. W. L., Wee, L., Rios Velazquez, E., Leijenaar, R. T. H., Parmar, C., Grossmann, P., Lambin, P. (2019). Data From NSCLC-Radiomics [Data set]. The Cancer Imaging Archive. https://doi.org/10.7937/K9/TCIA.2015.PFoM9REI

[16] P. Geurts, D. Ernst., and L. Wehenkel, "Extremely randomized trees", Machine Learning, 63(1), 3-42, 2006

[17] Menze, B.H., Kelm, B.M., Masuch, R. et al. A comparison of random forest and its Gini importance with standard chemometric methods for the feature selection and classification of spectral data. BMC Bioinformatics 10, 213 (2009).

https://doi.org/10.1186/1471-2105-10-213

[18] In J, Lee DK. Survival analysis: part II - applied clinical data analysis. Korean J Anesthesiol. 2019 Oct;72(5):441-457. doi: 10.4097/kja.19183. Epub 2019 May 17. PMID: 31096731; PMCID: PMC6781220. 


\section{Chapter 6}

An Approach toward Automatic Classification of Tumor Histopathology of Non-Small Cell Lung Cancer Based on Radiomic Features

Ravindra Patil, Geetha Mahadevaiah and Andre Dekker Jr.Tomography. 2016;2(4):374-377. doi:10.18383/j.tom.2016.00244 


\begin{abstract}
Non-small cell lung cancer (NSCLC) contributes to $85 \%$ of the lung cancer burden. The histology of the tumor (squamous cell carcinoma, large cell carcinoma, and adenocarcinoma and "not otherwise specified") has prognostic significance and it is therefore imperative to identify tumor histology for personalized medicine, but biopsies are not always possible and carry significant risk of complications. In this study we have used Radiomics, which provides an exhaustive number of informative features, to aid in diagnosis of tumor types in a noninvasive manner. Specifically, this study evaluates radiomic features of NSCLC to identify the histopathology of the tumor. We have included 317 subjects and classified the underlying tumor histopathology into its four main sub types. The performance of the current approach is $20 \%$ more accurate than an approach only considering volumetric and shape based features.
\end{abstract}

Key words: Lung cancer; Histology; Radiomics; Non-small cell lung cancer (NSCLC) 


\section{Introduction}

Non-small cell lung cancer accounts for $85 \%$ of all the lung cancers and it is the second most common cause of cancer in both men and women. Estimates by the American Cancer Society for 2016 are an incidence in the United States of 224,390 new cases of lung cancer (117,920 in men and 106,470 in women) and 158,080 deaths from lung cancer $(85,920$ in men and 72,160 in women). Every year, more people die of lung cancer than of colon, breast and prostate cancer combined. Two out of three people diagnosed with lung cancer are 65 or older, while less than $2 \%$ are younger than 45 years (1). The different factors that can affect survival include genetic factors, clinical factors such as age, and overall health, the size and stage of the tumor and the histological subtype of NSCLC. With regards to the subtype, many studies have identified a link between the subtype and survival. For example, Ma et al (2) recently showed that adenocarcinoma patients have a worse prognosis. Similarly, Yano et al (3) showed recently that squamous cell carcinoma have worse outcomes and concluded that the surgical management should be different for different subtypes. Given this knowledge, it is imperative to have histological classification of non-small cell-lung carcinoma. Currently, a biopsy is performed to identify the subtype which involves an invasive procedure and frequent sample extraction from the site of interest. Also, it is painful, costly and not without risk (4).

In recent years more emphasis has been given to non-invasive diagnosis and screening of lung cancer $(5,6)$. An advanced technique named "Radiomics" involving the extraction of large quantitative features, which results in conversion of images into higher dimensional minable data, can be used for building decision support systems. This is in contrast to the traditional practice of treating medical images as pictures used solely for visual interpretation. The output from a Radiomics analysis contains first, second, and higher-order statistics data derived from the entire image or a particular Region of Interest (ROI). It can be performed with tomographic images from CT, MR imaging, and PET studies [14]. The seminal work performed by Aerts et.al (7) showed radiomic features having prognostic power in lung and head-and-neck cancer patients. 
Also, there exist several studies in NSCLC by Al-Kadi et.al , Cook et.al and Ganeshan et.al (8-10) in which texture analysis is applied to predict lung cancer outcomes based on factors associated with tumor texture. In this work we hypothesize that radiomic texture features can classify the different subtypes of NSCLC in a noninvasive approach.

The aim of this study is to perform a radiomic analysis to identify tumor histopathology in NSCLC and classify into squamous cell carcinoma, adenocarcinoma, large cell carcinoma and not otherwise specified (NOS) non-small cell lung cancer. We extracted radiomic features and evaluated their ability to classify the tumor histopathology and also evaluated how these features compare with non-radiomic, "normal" features (e.g., volume, diameter).

\section{Methodology}

The applied methodology involves image acquisition, segmentation, feature extraction and model validation to identify the histology of the underlying tumor. Figure 1 summarizes the above mentioned approach. The approach involved was to characterize the tumor region through extraction of Radiomics features which earlier were thought to be redundant or non-useful for clinical outcome. The obtained CT image is segmented to define the tumor region and in this study Gross Tumor volume (GTV) is used. The segmented region is used to extract features based on tumor intensity, shape and texture. The extracted features are analyzed to build a decision support system to identify histopathology of the tumor.

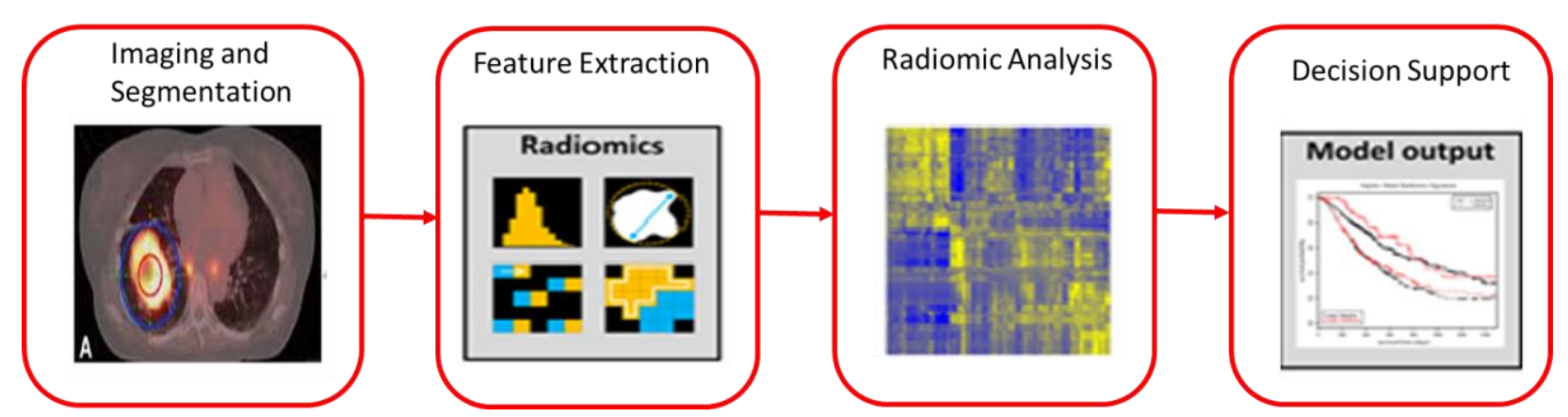

Fig. 1. The Radiomics workflow, images partly from (7) with permission 


\section{Data}

The data used in this study were obtained from The Cancer Imaging Archive (TCIA) (http://www.cancerimagingarchive.net/) from the collection of NSCLC-Radiomics. In all 317 patient CT data having NSCLC cancer were considered out of which 40 were Adenocarcinoma, Large cell carcinoma - 108, Squamous cell carcinoma - 110 and "not otherwise specified" were 59. The demographic distribution is shown in Table 1. The ground truth of the tumor region was provided by the radiation oncologist in the DICOM-RTSTRUCT file and hence no explicit segmentation algorithm was used for the tumor region identification. The data was not preprocessed or normalized so as not to devalue any of the clinical information present in the images.

\begin{tabular}{|l|c|c|c|c|}
\hline $\begin{array}{l}\text { Subject } \\
\text { Characteristics }\end{array}$ & Adenocarcinoma & $\begin{array}{c}\text { Large cell } \\
\text { carcinoma }\end{array}$ & $\begin{array}{c}\text { Squamous } \\
\text { cell } \\
\text { carcinoma }\end{array}$ & NOS \\
\hline $\begin{array}{l}\text { Number of } \\
\text { subjects }\end{array}$ & 40 & 108 & 110 & 59 \\
\hline Male & 20 & 65 & 70 & 41 \\
\hline Female & 20 & 43 & 40 & 18 \\
\hline $\begin{array}{l}\text { Mean Age } \\
\text { (years) }\end{array}$ & 67.2 & 66.9 & 70.2 & 65.6 \\
\hline
\end{tabular}

Table 1. Distribution of the subject characteristics

\section{Feature extraction}

An important sub step of Radiomics is the high throughput extraction of quantitative image features. In total 431 imaging features were extracted from the site of the tumor identified by the manual delineation performed by the radiation oncologist. The feature extraction algorithm was custom implemented in Matlab. The quantitative imaging features were divided into four sub groups namely (i) First order statistics (ii) Shape and size based features (III) Textural features (IV) Multiscale wavelet; which describe tumor 
phenotypes. First-order statistics provides a distribution of voxel intensities within the CT image through commonly used and basic metrics (e.g. energy, entropy, kurtosis).

The shape and size based features provide tumor compactness, volume, area metrics along with how spherical, rounded or elongated the tumor is.

The first order statistics represent only the information related to the gray level distribution and do not provide pertinent information on the relative position of various gray level across images. To capture this information, we computed textural features using gray level co-occurrence (GLCM) and gray level run-length (GLRLM) texture matrices. All the voxel intensities were resampled into equally spaced bins using a width of 25 Hounsfield Units. The former step reduces image noise as well as normalizes the intensities and will allow direct comparison of all calculated textural between patients. The matrices were determined considering 26 - connected voxels in all 13 directions in 3D.

The wavelets features help to decouple original images into high and low frequencies. In this analysis, we applied one level un-decimated 3-D wavelet transform on the GTV. The original image is decomposed into 8 level of wavelet decomposition $\mathrm{X}_{\mathrm{LLL}}, \mathrm{X}_{\mathrm{LLH}}$, $\mathrm{X}_{\mathrm{LHL}}, \mathrm{X}_{\mathrm{LHH}}, \mathrm{X}_{\mathrm{HLL}}, \mathrm{X}_{\mathrm{HLH}}, \mathrm{X}_{\mathrm{HHL}}$ and $\mathrm{X}_{\mathrm{HHH}}$ ), where $\mathrm{L}$ and $\mathrm{H}$ are low pass and high pass. For example, $\mathrm{X}_{\mathrm{HLH}}$ is interpreted as the high-pass sub band resulting from directional filtering in $\mathrm{x}$ with high pass, a low pass along $\mathrm{y}$ - direction and high pass in $\mathrm{z}$-direction.

$$
X_{L L H}(i, j, k)=\sum_{p=1}^{N_{H}} \sum_{q=1}^{N_{L}} \sum_{r=1}^{N_{H}} H(p) L(q) H(r) X(i+p, j+q, k+r)
$$

Where $N_{H}$ the length of filter $\mathrm{H}$ and $N_{L}$ is the length of filter $\mathrm{L}$.

For each of the decompositions obtained above we computed first order statistics and textural features as mentioned earlier. The feature vector length for each of the subjects is 431 . 
The first order statistics accounts to 14 features, shape and size based features were 8 , textural features accounted to 33 and remaining 376 features were derived from wavelet decomposition.

\section{Classification}

As the classes of data were skewed and in order to eliminate the bias in learning due to unequal samples in each class, the SMOTE algorithm (11) was applied on the dataset. Two data models were built in this experiment, the first being called radiomic model contained all the radiomic features extracted in the above step and the second being the normal model consisting of typical normal features which were subset of radiomic features consisting of (Energy, Entropy, Kurtosis, Maximum, Mean, Mean absolute deviation, Median, Minimum, Range, RMSvalue, Skewness, Standard Deviation, Uniformity, Variance, Compactness, Maximum 3d diameter, Spherical disproportion, Sphericity, Surface Area, Surface to volume ratio, Volume). Following which, a multicategory support vector machine using $\mathrm{R}$ with package (e1071) [13] was used for classifying the data into predefined classes.

The results obtained were based on 10 fold cross validation on the dataset. The experimental parameters set for SVM were kernel type: rbf, gamma: 1.o, C: o.1, eplison: $10^{\wedge}(-4)$. The selection of the above mentioned hyper parameters were performed based on a grid search to yield the best accuracy value. Furthermore, the ranking of features based on the built model was performed to identify the most important predictive features that aided in the classification using the Caret package in R. 


\section{Results}

The demographic of the dataset consist of both male (6o\%) and female (40\%) subjects. The average age of the pool was 68 years. The metrics of the histology classification obtained by considering Radiomics features and that of normal features are shown in the Figure 2. It can be observed that there is a 20 percent improvement $(p<1.2 e-4)$ over the accuracy in identification of the tumor histopathology using radiomic features compared to normal features.

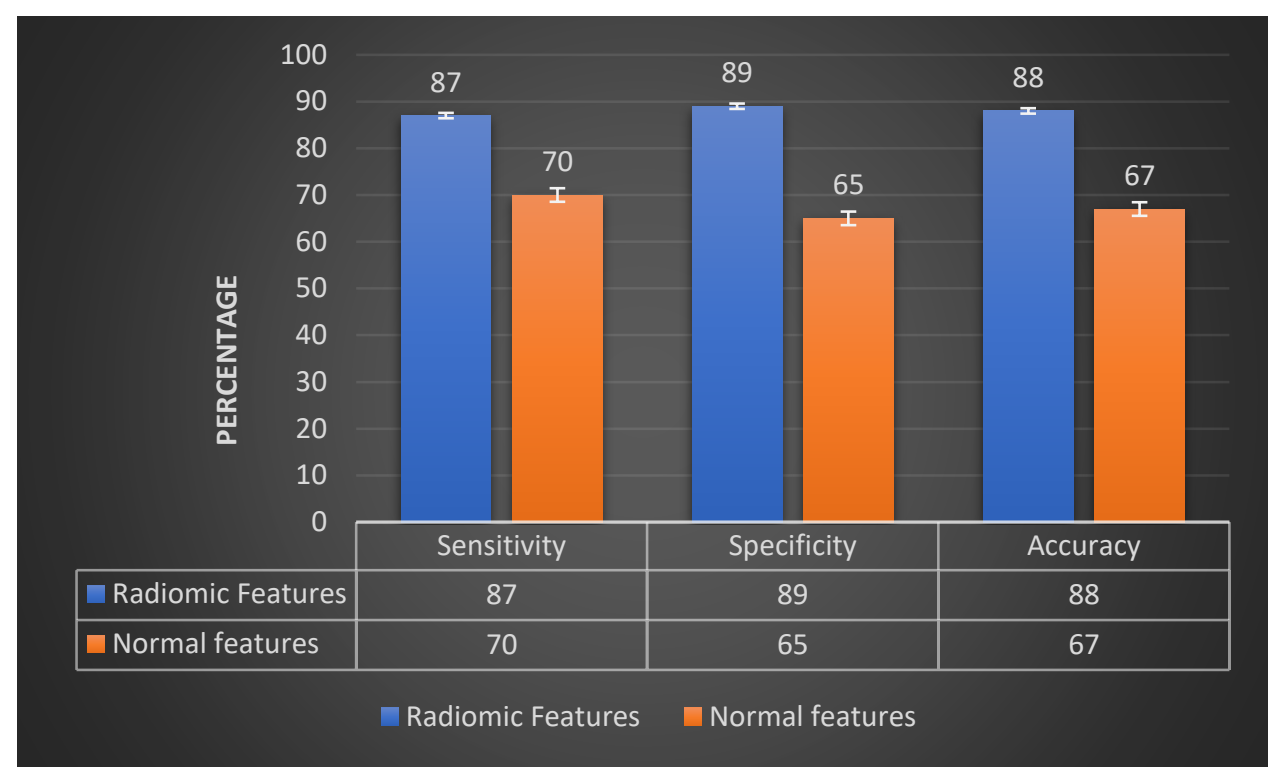

Fig.2. Classification metrics using Radiomic and Normal features

Also, feature ranking was employed on the radiomic model to rank the features based on the importance value. The top 40 radiomic features which aid in the classification of the tumor histopathology are shown in Figure 3. It is interesting to note that volume as an independent feature doesn't rank as the top contributor for the classification. Also, wavelet based features dominate the top contributing features for the classification, strengthening the claim that additional features aid in better information extraction. Hence, Radiomics is able to quantify phenotypical differences from medical images by using a large set of imaging features and providing more hidden information compared to normal features in classification of tumor histopathology. 


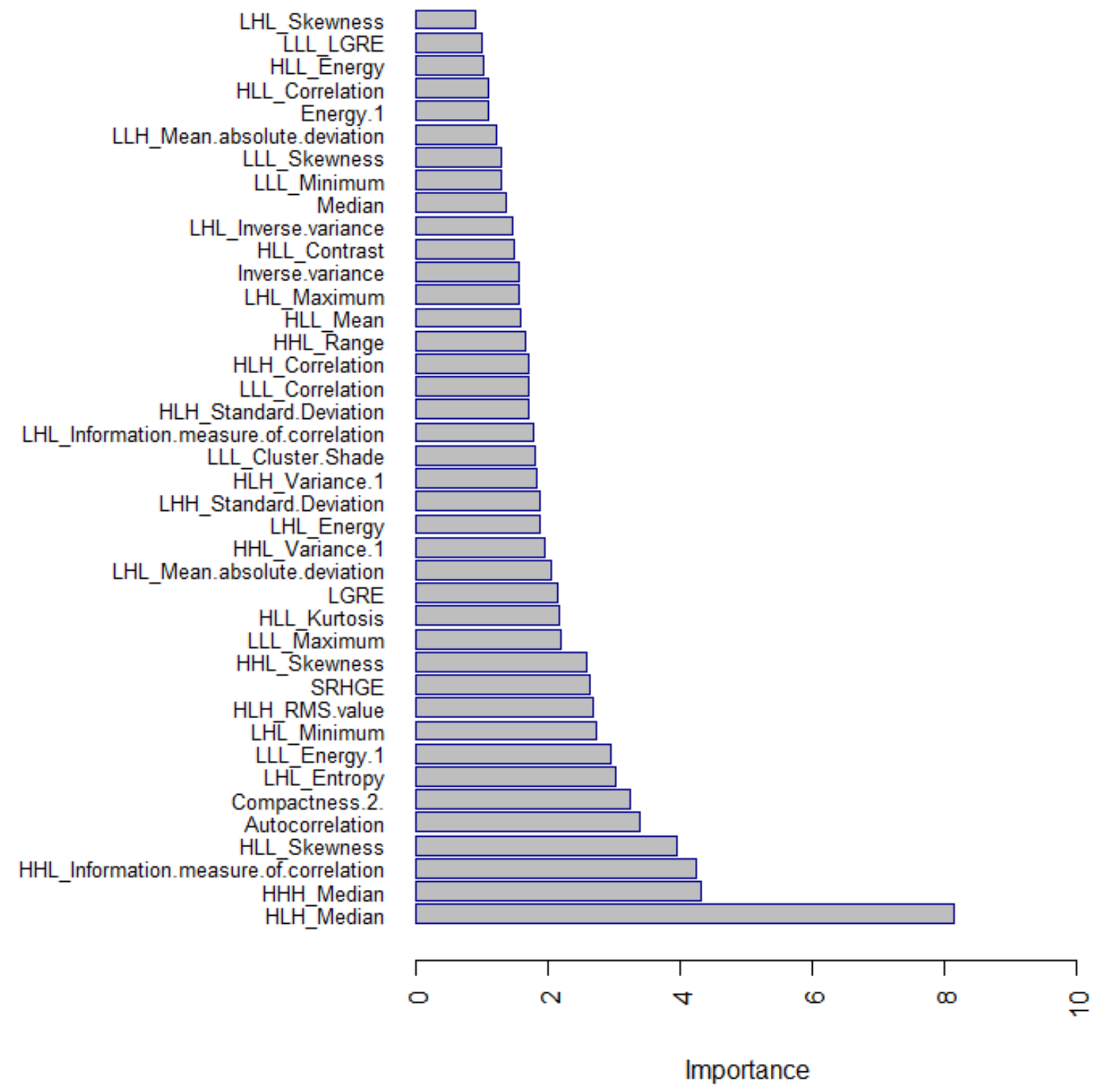

Figure. 3 Radiomic features ranking based on importance

\section{Discussion}

In this study we have adapted Radiomics to classify NSCLC tumors according to their histopathology namely adenocarcinoma, squamous cell carcinoma, large cell carcinoma and not-otherwise-specified by extracting imaging features from annotated tumor region. The results of the study show that Radiomics has greater potential than normal features in classifying the tumor histopathology. Our approach provides a noninvasive, 
fast, low cost and repeatable way of investigating tumor histology, hence speeding up the development of personalized medicine. However, our method directly considers the tumor region segmented by the radiation oncologist, in future our work will be focused on integrating automatic segmentation in the workflow. Furthermore, the limitations of this study are a lack of validation by an external dataset and that we did not compare it to non-invasive liquid biopsies (12), which might also provide a method to determine histology although this is still quite an experimental technique.

Conflict of Interest: None reported.

\section{References}

[1]. A report on Lung Cancer (Non-Small Cell), American Cancer Society, 2016 (http://www.cancer.org/acs/groups/cid/documents/webcontent/oo3115-pdf.pdf).

[2]. Ma LH, Li G, Zhang HW, Wang ZY, Dang J. The effect of non-small cell lung cancer histology on survival as measured by the graded prognostic assessment in patients with brain metastases treated by hypofractionated stereotactic radiotherapy. Radiat Oncol. 2016 Jul 13;11:92. doi: 10.1186/s13014-016-0667-X.

[3]. Yano M, Yoshida J, Koike T, Kameyama K, Shimamoto A, Nishio W, Yoshimoto K, Utsumi T, Shiina T, Watanabe A, Yamato Y, Watanabe T, Takahashi Y, Sonobe M, Kuroda H, Oda M, Inoue M, Tanahashi M, Adachi H, Saito M, Hayashi M, Otsuka H, Mizobuchi T, Moriya Y, Takahashi M, Nishikawa S Matsumura Y, Moriyama S, Fujii Y. The Outcomes of a Limited Resection for Non-Small Cell Lung Cancer Based on Differences in Pathology. World J Surg. 2016 Jun 30. doi:10.1007/soo268-016-3596-9.

[4]. Wu CC1, Maher MM, Shepard JA. Complications of CT-guided percutaneous needle biopsy of the chest: prevention and management. AJR Am J Roentgenol. 2011 Jun;196(6):W678-82. doi: 10.2214/AJR.10.4659.

[5]. Bajtarevic A1, Ager C, Pienz M, Klieber M, Schwarz K, Ligor M, Ligor T, Filipiak W, Denz H, Fiegl M, Hilbe W, Weiss W, Lukas P, Jamnig H, Hackl M, Haidenberger A, Buszewski B, Miekisch W, Schubert J, Amann A. Noninvasive detection of lung cancer by analysis of exhaled breath. BMC Cancer. 2009 Sep 29;9:348. doi: 10.1186/1471-2407-9-348.

[6]. D'Urso V, Doneddu V, Marchesi I, Collodoro A, Pirina P, Giordano A, Bagella L. Sputum analysis: non-invasive early lung cancer detection. J Cell Physiol. 2013 May;228(5):94551. doi: $10.1002 /$ jcp.24263.

[7]. Hugo J. W. L. Aerts, Emmanuel Rios Velazquez, Ralph T. H. Leijenaar, Chintan Parmar, Patrick Grossmann, Sara Carvalho, Johan Bussink, René Monshouwer, Benjamin HaibeKains, Derek Rietveld, Frank Hoebers, Michelle M. Rietbergen, C. René Leemans, Andre Dekker, John Quackenbush, Robert J. Gillies \& Philippe Lambin. Decoding tumour phenotype by noninvasive imaging using a quantitative Radiomics approach. Nature Communications 5, Article number: 4006 (2014) doi:10.1038/ncomms50o6.

[8]. Al-Kadi OS, Watson D. Texture analysis of aggressive and nonaggressive lung tumor CE CT images. IEEE Trans Biomed Eng. 2008; 55(7):1822-1830. doi: 10.1109/TBME.2008.919735. 
[9]. Gary J.R. Cook, Connie Yip, Muhammad Siddique, Vicky Goh, Sugama Chicklore, Arunabha Roy, Paul Marsden, Shahreen Ahmad, David Landau. Are Pretreatment 18FFDG PET Tumor Textural Features in Non-Small Cell Lung Cancer Associated with Response and Survival After Chemoradiotherapy? J Nucl Med 2013; 54:1-8 doi: 10.2967/jnumed.112.107375.

[10]. Ganeshan B, Goh V, Mandeville HC, Ng QS, Hoskin PJ, Miles KA. Non-small cell lung cancer: histopathologic correlates for texture parameters at CT. Radiology. 2013 Jan;266(1):326-36. doi: 10.1148/radiol.12112428. Epub 2012 Nov 20.

[11]. Nitesh V. Chawla, Kevin W. Bowyer, Lawrence O. Hall, W. Philip Kegelmeyer. SMOTE: Synthetic Minority Over-sampling Technique,SMOTE: Synthetic Minority Oversampling Technique. Journal of Artificial Intelligence Research 16 (2002) 321-357.

[12]. Graham Brock, Elena Castellanos-Rizaldos, Lan Hu, Christine Coticchia, Johan Skog. Liquid biopsy for cancer screening, patient stratification and monitoring. Transl Cancer Res 2015;4(3):280-290 .

[13] https://cran.r-project.org/web/packages/e1071/e1071.pdf

[14] Jun Wang, Xia Liu, Di Dong, Jiangdian Song, Min Xu, Yali Zang, Jie Tian. Prediction of malignant and benign lung tumor using a quantitative radiomic method. $38^{\text {th }}$ Annual International Conference of IEEE Engineering in Medicine and Biology Society (EMBC), 2016. 


\section{Chapter 7}

Fractal Analysis in Histology Classification of Non-Small Cell Lung

Cancer

Ravindra Patil, Geetha Mahadevaiah, Srinidhi Bhat, Dinesh M.S, Leonard Wee and Andre Dekker

\section{Medical Imaging}

Artificial Intelligence, Image Recognition, and Machine Learning Techniques, 2019, pp. 64-75 DOI: https://doi.org/10.1201/9780429029417 


\begin{abstract}
Non-small cell lung cancer (NSCLC) accounts for $85 \%$ of all lung cancers. Noninvasive identification of the histology of NSCLC might aid in determining the appropriate treatment approaches. In this study, we have studied the use of Radiomics with the application of fractals to arrive at histology classification of NSCLC using lung CT images. This study suggests that fractals can play a vital role in Radiomics, providing information not only about the Gross Tumor Volume (GTV) structure, but also can help in characterization of the tumor. It was observed that fractal dimension based features were among the top 15 features that aided in histology classification based on 317 subjects and improved the existing classification accuracy of the NSCLC histology by $8 \%$.
\end{abstract}

Keywords: Histology, NSCLC, Fractal Dimension 


\section{Introduction}

In both sexes combined, lung cancer is the most commonly diagnosed cancer (11.6\% of the total cases) and the leading cause of cancer death. The total number of lung cancer cases in 2018 alone amounted to 2,093,876 with the number of deaths with lung cancer being 1,761,007. Nonsmall cell lung cancer (NSCLC) accounts for $85 \%$ of all lung cancers [1]. The cause and the survival of NSCLC subject varies across age, genetic profile, size of tumor and histopathology of tumor. There are various studies that have established a correlation between the subtypes of NSCLC (squamous cell carcinoma, large cell carcinoma, and adenocarcinoma and "not otherwise specified") to that of survival. Specifically, it was observed that the adenocarcinoma prognosis is poor compared to those of non-adenocarcinoma [2]. It was also concluded that the surgical management should be different for each sub categories of NSCLC [3]. The current approach of sub type detection is performed using a biopsy procedure, where the tissue under observation is biopsied to determine the subtype, which is invasive in nature. The invasive approach is painful, costly and also not devoid of complications [4]. Recently, several studies have been undertaken to identify the sub categories of NSCLC non-invasively using an approach of Radiomics, wherein a large amount of quantitative features are mined and decision support models are built to achieve the desired objective [5]. Radiomics has also been applied to several other medical challenges in tumors of the lung, breast and prostate. Radiomics has also been applied on images extracted from different medical imaging modalities, computed tomography (CT), magnetic resonance (MR), and positron emission tomography (PET) [6-9, 16-19], showing promising results in each case.

Also, there has been lot of interest in application of fractals in the oncology domain. Fractals are mathematical objects which have a non-integer dimension. These objects manifest repeating pattern at different size scales; this property is quantified by a parameter named fractal dimension that measures the self-similarity grade of the structure under analysis [10]. Such mathematical objects can be self-similar and resembles the repeated pattern within itself. These patterns have been studied in the oncology domain to differentiate between malignant and benign tumors in case of breast cancer. Comprehensive reviews on the use of fractal Prognostic and Prediction Modelling with Radiomics for Non-Small Cell Lung Cancer | 123 
dimensions exist in various medical research areas such as pathology [11 -12]. Recent trends show fractals to be a useful measure of the pathologies of the vascular architecture, tumor/parenchymal border, and cellular/nuclear morphology. A procedure that combines fractal and segmentation analysis has been proposed to investigate heterogeneity in cancer cells on MR images, similar to the approach described by Szigeti et al.,[20] for studying lung tumor heterogeneity on mice CT scans. More details on the fractals and its applications in oncology can be found in [11].

Other major studies include different fractal measures such as the power-law behavior of the Fourier spectrum of gray-scale images, which was used by Heymans et al. [13] to characterize the microvasculature in cutaneous melanoma. The fractal dimension quantified the degree of randomness in the vascular distribution, a characteristic that cannot be easily captured by the vascular density. Another study by Cusumano et al. [14][21] aimed to use a fractal based radiomic approach to predict complete pathological response after chemo-therapy in locally advanced rectal cancer (LARC). Fractals played an important role giving information not only about the gross tumor volume (GTV) structure but also describing information about the subpopulations in the GTV [14].

In this study, we investigate the role of fractals in the classification of Non-Small Cell Lung Cancer histology. We follow the box counting approach that aims to overlay boxes of various sizes over the area of interest and to optimize this quantity with the size of each box. Overall, our technical contributions are:

1) Building an algorithm which can compute the fractal dimension of a two dimensional region of interest in a given volume.

2) Analyzing the extent to which fractal dimension aids in classification of NSCLC subtypes in the presence of other Radiomic features.

\section{Methodology}

The methodology adapted in this study consists of acquisition of the NSCLC images with various histology subtypes (i.e. squamous cell carcinoma, large cell carcinoma, and adenocarcinoma and "not otherwise specified"), segmentation of the GTV, computation of 
fractals, extraction of Radiomic features and validation of the model to identify the histology of the underlying tumor. The pictorial depiction of the workflow is shown in the Figure 1.

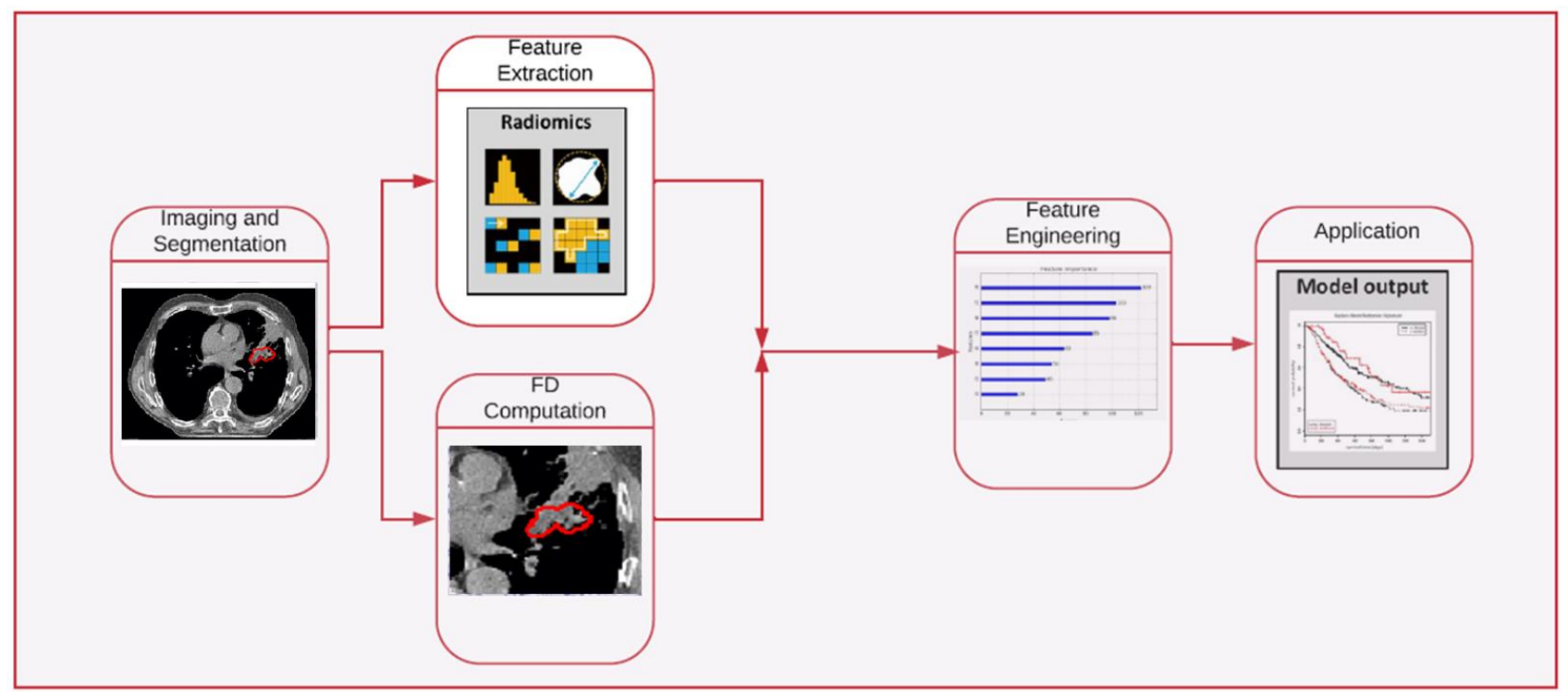

Figure 1. Workflow and Approach adapted. FD = Fractal dimension.

\section{Image Analysis}

The following experiment involved Computerized Tomography (CT) Images of NSCLC. Images of 317 patients were used in this study and the data set was obtained from the (http://www.cancerimagingarchive.net/) [9] from the collection of NSCLC-Radiomics. The images were in Digital Imaging and Communications in Medicine (DICOM) format and the CT image of each patient had the corresponding Structure Set file (RTSTRUCT) which included the GTV delineation performed by an expert radiation oncologist. The distribution of the demographic of data is depicted in the Table 1.

\begin{tabular}{|l|c|c|c|c|}
\hline $\begin{array}{l}\text { Subject } \\
\text { characteristics }\end{array}$ & Adenocarcinoma & $\begin{array}{c}\text { Large cell } \\
\text { carcinoma }\end{array}$ & $\begin{array}{c}\text { Squamous } \\
\text { cell } \\
\text { carcinoma }\end{array}$ & NOS \\
\hline $\begin{array}{l}\text { Number of } \\
\text { subjects }\end{array}$ & 40 & 108 & 110 & 59 \\
\hline Male & 20 & 65 & 70 & 41 \\
\hline
\end{tabular}




\begin{tabular}{|l|c|c|c|c|}
\hline Female & 20 & 43 & 40 & 18 \\
\hline Mean Age (years) & 67.2 & 66.9 & 70.2 & 65.6 \\
\hline
\end{tabular}

Table 1. Subject Demographics vs NSCLC histology subtype. NOS=Not Otherwise Specified

Accessing the DICOM tags which contained the contour information of the Gross Tumor Volume (GTV) using the information in the RTSTRUCT file, we create a mask depicting the GTV. The extracted mask was superimposed on the actual image to delineate the region of interest. Further, the minmax normalization of the images was performed to ensure the effect due to spike pixels are minimized [22].

\section{Computation of Fractal Dimension}

The fractal dimension was computed based the box counting approach and was further optimized to deduce the fractal dimension for each region of interest. In our approach we extracted every surface contour of the GTV and box counting algorithm was applied to compute fractal dimension on each of the slice containing the tumor volume. The pictorial representation of the approach can be seen in the Fig 2 with varied grid sizes [15]

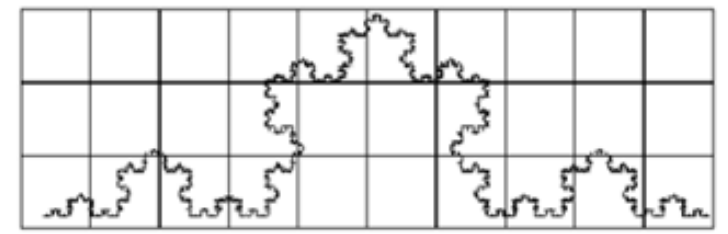

The Koch curve with unit 1 grid size, with 18 containing the curve.

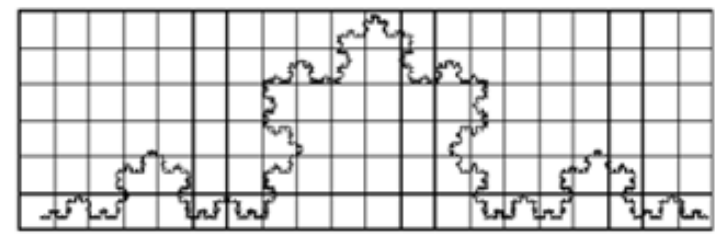

The Koch curve with unit $1 / 2$ grid size, with 41 containing the curve.

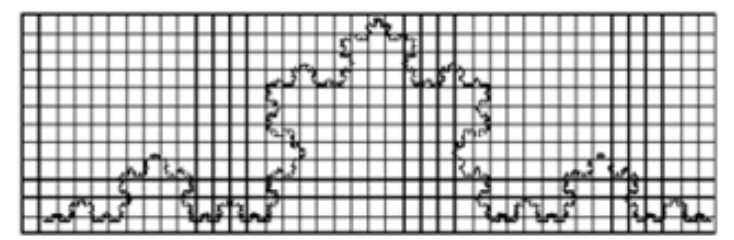

The Koch curve with unit $1 / 4$ grid size, with 105 containing the curve.

Figure 2. Sample representation of box counting approach with varying N [15]

126 | Prognostic and Prediction Modelling with Radiomics for Non-Small Cell Lung Cancer 
In this study the fractal dimension (FD) was computed on the GTV on the CT slices. The equation for the computation is mentioned in (1).

$$
F D=\frac{\log (N)}{\log \left(\frac{1}{r}\right)}
$$

Where:

FD is the Fractal Dimension

$\mathrm{N}$ is the number of boxes needed to cover the region of interest $r$ is the size of each box

The above equation is recursively applied by varying the size of the box, there by converting it into a curve fitting solution. In principle, this corresponds to a line fitting problem, where points corresponding to the $\mathrm{N}$ and $\mathrm{l} / \mathrm{r}$ are fit and the slope of the line provides the fractal dimension. This is one of the reasons we have applied a logarithm to equation (1). This reduces a curve fitting method to a line fitting method, which is computationally simpler to solve. Also, a logarithm, being a monotonic function in nature does not alter the behavior of the original equation. The FD value for sample subsets are shown in the Figure 3, which is computed based on each of the slices. 
Slice number 65 and FD is 0.31

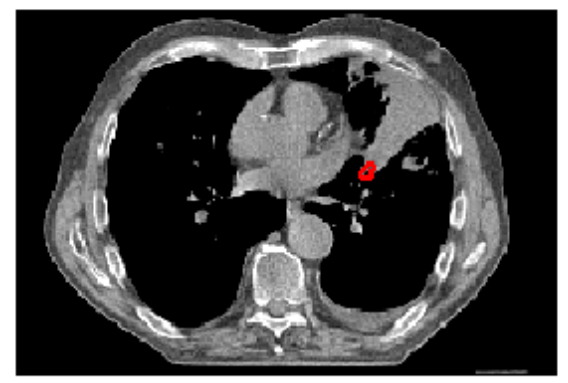

Slice number 67 and FD is 0.76

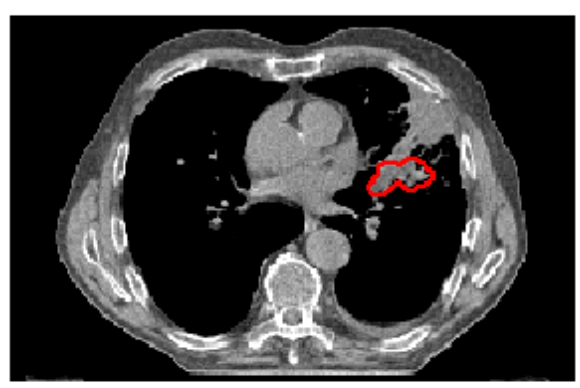

Slice number 69 and FD is 0.83

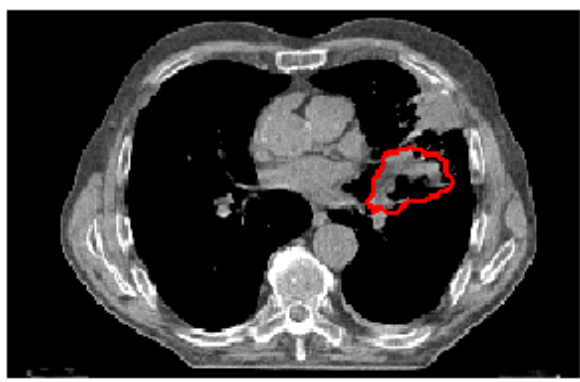

Slice number 66 and FD is 0.51

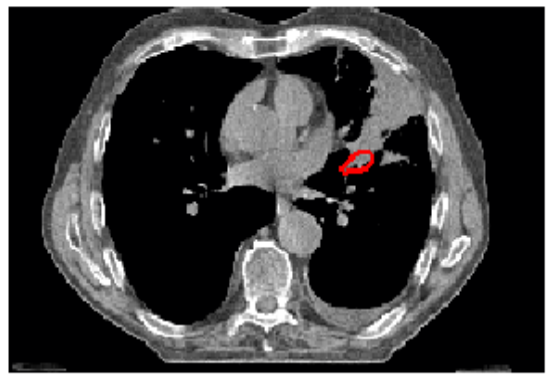

Slice number 68 and FD is 0.86

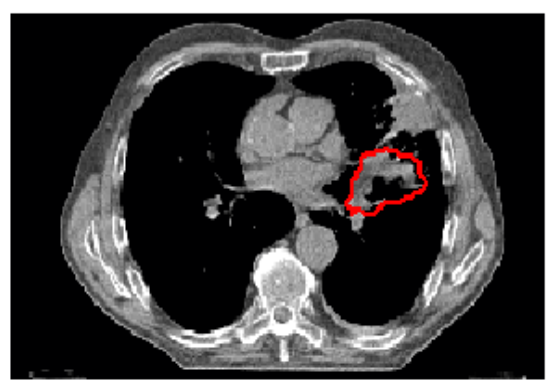

Slice number 70 and FD is 0.84

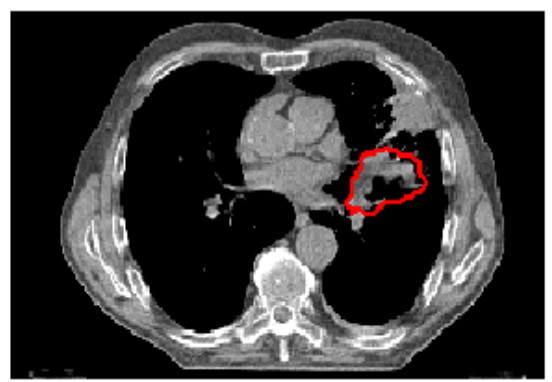

Figure 3. Subject Lungoo1 from the NSCLC-Radiomics data set with FD computed value

\section{Extraction of Radiomics Features}

The quantitative image features were extracted from the GTV, wherein these imaging features where divided into four sub categories (1) First order statistics (2) Textural Features (3) Shape and Size based features (4) wavelet features. The first order features provides the voxel intensity distribution within GTV. The textural features were computed using gray-level cooccurrence and gray-level run-length texture matrices, which aid in providing the relative position of various gray level distribution. Shape and size features provide information on how spherical, elongated or rounded the tumor manifests and provide information about area, tumor compactness and volume. The wavelet features provide information by decoupling the region into high and low frequencies with GTV as input. In this approach, the original images 128 | Prognostic and Prediction Modelling with Radiomics for Non-Small Cell Lung Cancer 
are decomposed into 8 levels of wavelet decompositions $\left(\mathrm{X}_{\mathrm{LLL}}, \mathrm{X}_{\mathrm{LLH}}, \mathrm{X}_{\mathrm{LHL}}, \mathrm{X}_{\mathrm{LHH}}, \mathrm{X}_{\mathrm{HLL}}, \mathrm{X}_{\mathrm{HLH}}, \mathrm{X}_{\mathrm{HHL}}\right.$ and $\mathrm{X}_{\mathrm{HHH}}$ ), where $\mathrm{L}$ and $\mathrm{H}$ are low pass and high pass. For example, $\mathrm{X}_{\mathrm{LHL}}$ is interpreted as the low-pass sub band resulting from directional filtering in $\mathrm{x}$ with low pass, a high pass along $\mathrm{y}^{-}$ direction and low pass in z-direction.

$X_{L L H}(i, j, k)=\sum_{p=1}^{N_{H}} \sum_{q=1}^{N_{L}} \sum_{r=1}^{N_{H}} H(p) L(q) H(r) X(i+p, j+q, k+r)$

Where $N_{H}$ the length of filter $\mathrm{H}$ and $N_{L}$ is the length of filter L.

In total 431 Radiomics features were extracted and these formed the feature vector for each of the subjects. Further, to this feature vector, Max FD, average FD obtained from the fractal dimension computation were augmented making it a 433 feature vector for each subject.

\section{Classification}

Two classification models were built in this experiment, one with all radiomic features including fractal features extracted and the second with only radiomic features excluding the fractal features. A Random Forest Classifier (RFC) was used to model the multiclass classification problem of predicting the histology of the tumor into one of the following sub categories: Squamous cell carcinoma, Large cell carcinoma, Adenocarcinoma and not otherwise specified. The RFC was implemented using sklearn ensemble package in python and to obtain the best set of parameters we defined a grid of hyper-parameter ranges using ScikitLearn's RandomizedSearchCV package by performing 10-fold cross validation with each combination of values.

The hyper parameters and the values of the RFC tuned in this experiment were:

- max_features: This parameter describes the maximum number of features the random forest is allowed to try in an individual tree. The value in this experiment was chosen as "auto" which will take all the features in every tree. Though this option decreases the speed of the algorithm, it provides a high number of options to be considered at each 
node. Moreover, in this experiment we have first chosen the top 15 contributing features and then used them for the classification task, hence the sample space is reduced compared to the original 433 .

- n_estimators: This parameter describes the number of trees to be built before taking the maximum voting of the predictions. Empirically, in this experiment, the optimal value for this parameter has been 10.

- Criterion: This parameter describes the criteria of split. In this experiment we have used the Gini index impurity measure.

- max_depth: This parameter represents the depth of each tree in the forest. The deeper the tree, the more splits it has and the more information it can capture regarding the data. In this case the optimal depth value was found to be 15 .

- min_samples_leaf: This describes the minimum samples required to be at the leaf node. In this experiment the optimal value of parameter is found to be 3 .

\section{Results}

There were a total of 317 subjects that were considered for the histology classification, out which $40 \%$ of the subjects were female and the average age was 68 years. A Pearson's correlation analysis was performed to understand the relationship of first order Radiomics and FD features with that of the histology class. It can be observed that max FD has maximum correlation with respect to histology class compared to other first order features. Further, it can also be seen that tumor volume as an independent feature ranks much lower than the FD in Figure 4. This is in line with the understanding that the morphology of the tumor provides a vital distinction other than tumor volume in histology differentiation. 


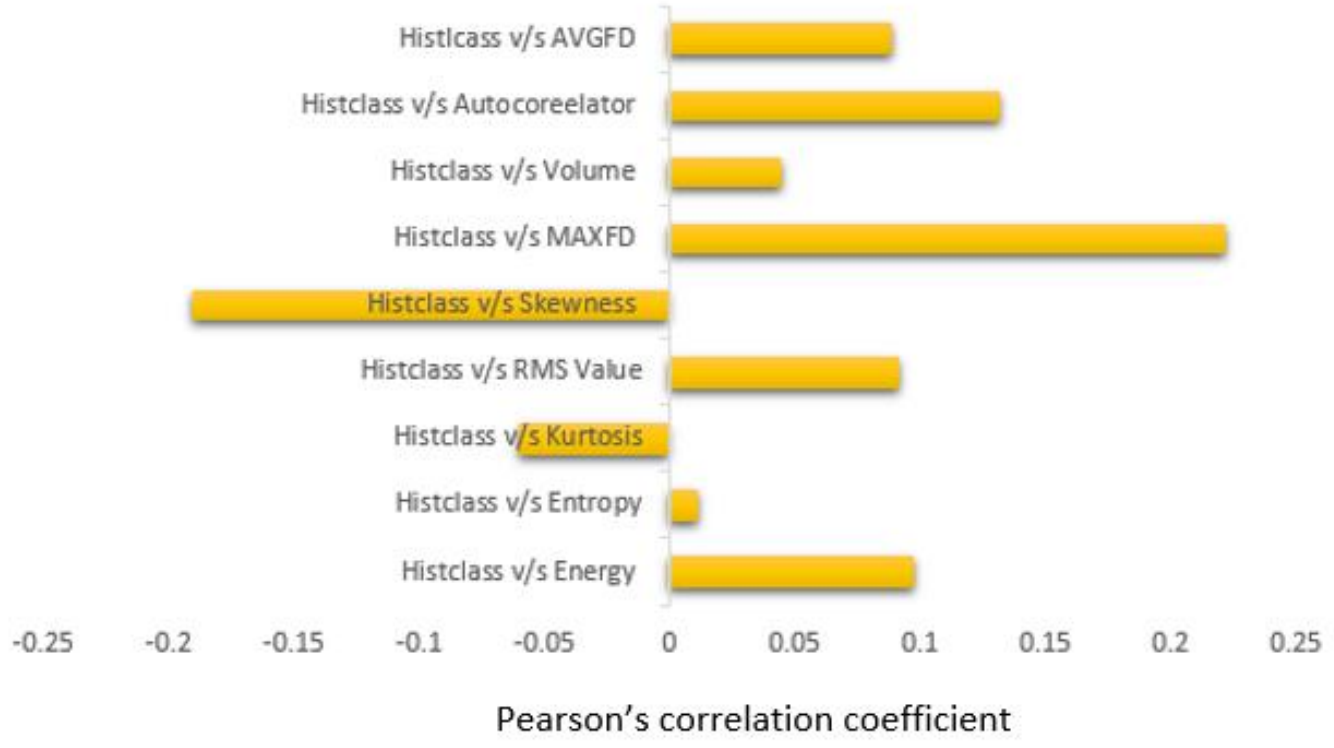

Figure 4. Correlation of Histology with various first order Radiomics features and FD features.

HistClass=Histology Class, $A$ VGFD=Average Fractal Dimension; RMS value= Root Means Square; MAX FD= Maximum Fractal Dimension

The classification accuracy considering the Radiomics features accounted for $74 \%$ however with inclusion of FD features it was improved to $86 \%(\mathrm{P}<0.001)$. Also, there is an improvement of $8 \%$ and $17 \%$ in terms for sensitivity and specificity respectively by considering the FD features (Figure 5).

Prognostic and Prediction Modelling with Radiomics for Non-Small Cell Lung Cancer $\mid 131$ 


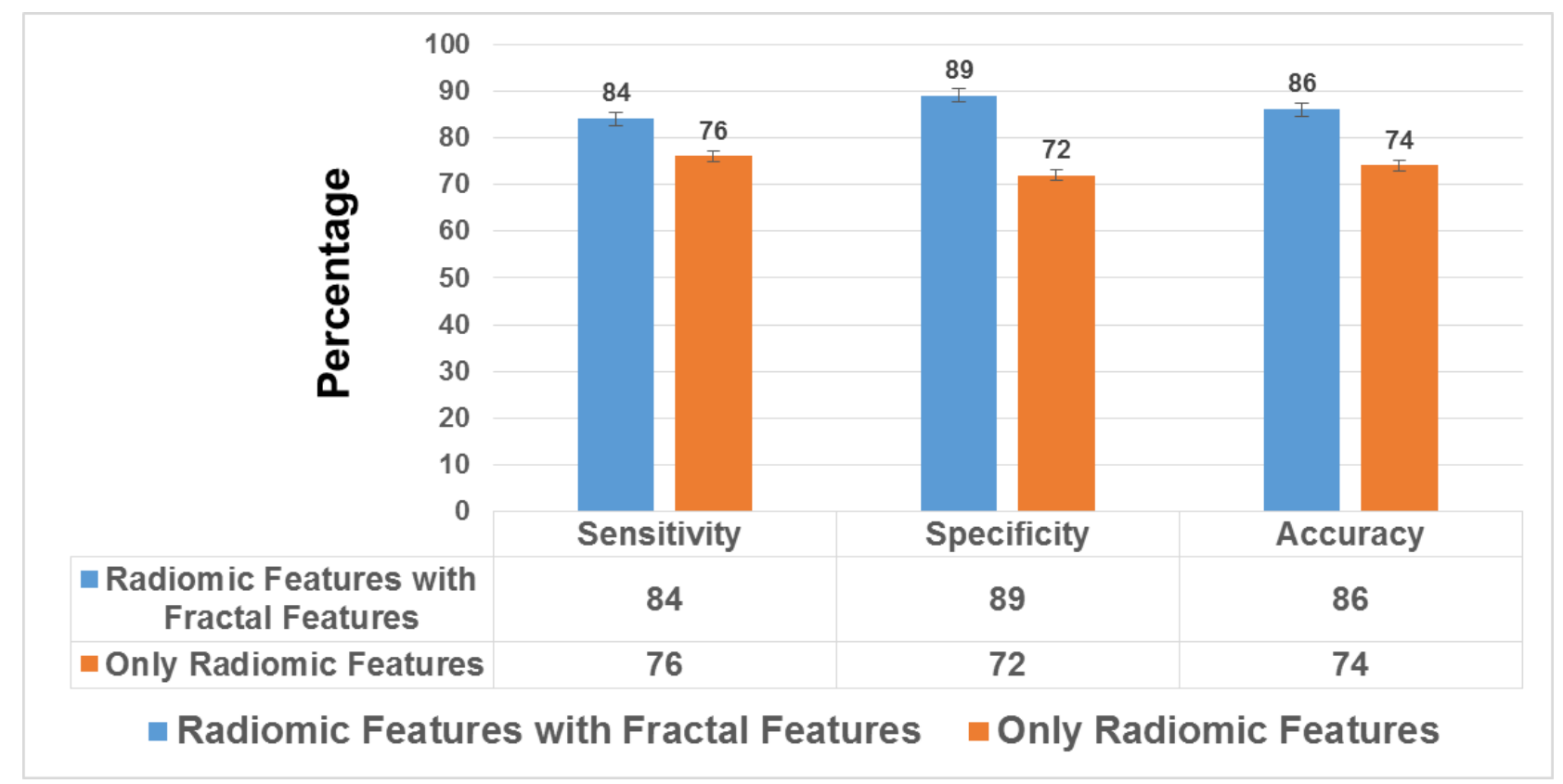

Figure 5. Classification metric comparison between with FD and without FD features inclusion

Furthermore, the features were ranked based on a random forest classifier on the priority of importance for classification of histology as shown in Figure 6. The top features contributing toward histological classification using radiomic features include HHH_Sum Entropy, HHH_LRGE, HHH_RLN, Inverse Difference Moment Normalized (IDMN), HHH_GLN, HHH_SRHGE, HHH_IDMN, Informational measure of correlation 2 (IMC2), Maximum FD, Average FD, HHH_Sum Variance. Also, it is interesting to note that the FD derived features, Max FD and Avg FD features were ranked among the top 15 features that aids in histology classification of NSCLC. In essence, wavelet-based features and FD parameters dominate as top contributing features for the histology classification. 


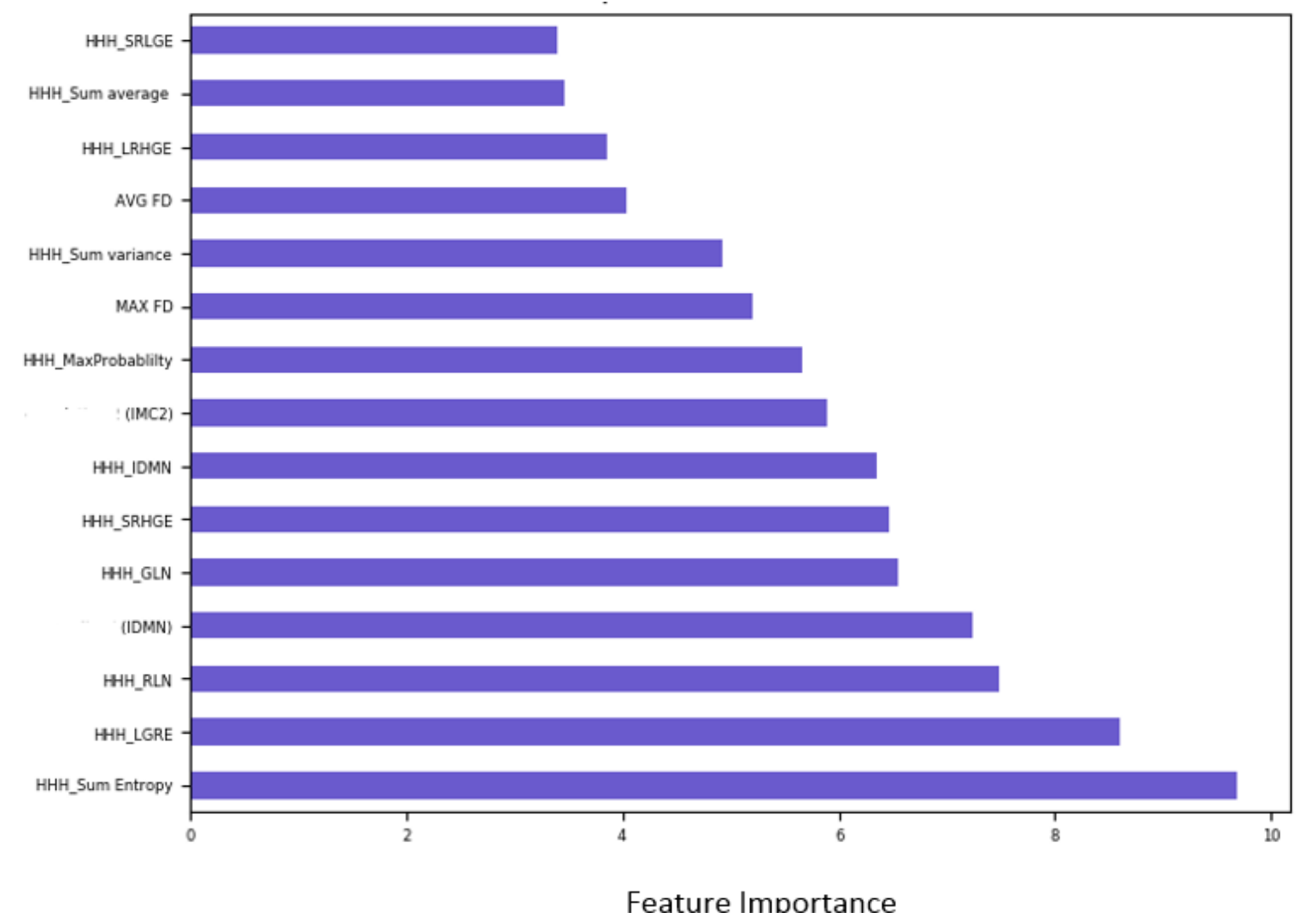

Figure 6 . Feature ranking based on the importance

\section{Conclusion}

In this study, we established that the fractal derived features play an important role in histology classification of NSCLC. Applying fractal analysis on a 2-D contour region can provide valuable information and can reflect the idea of overall tumor aggressiveness. However, our study has the following limitations: The fractal computation has been applied on 2-D contour GTV regions, this study could be extended to $3 \mathrm{D}$ fractal analysis algorithm using a mesh approach, so that the region of GTV is better delineated in $3 \mathrm{D}$ to capture heterogeneity and the irregularity of the underlying voxels leading to improved approach for computation of FD. Also, for a tumor contour which is very small, there might not be enough points in the number of boxes $\mathrm{v} / \mathrm{s}$ the size of each box plane $(\log (\mathrm{N})$ vs $\log (1 / \mathrm{r}))$ to describe the tumor contour. In this case, the best fit line will be only an approximation and hence this will be reflected in the FD value as well. 


\section{References}

[1] Freddie Bray et.al Global Cancer Statistics 2018: GLOBOCAN Estimates of Incidence and Mortality Worldwide for 36 Cancer in 185 Countries, CA CANCER J CLIN 2018;0:1-31

[2] Ma LH, Li G, Zhang HW, Wang ZY, Dang J, Zhang S, Yao L. The effect of nonsmall cell lung cancer histology on survival as measured by the graded prognostic assessment in patients with brain metastases treated by hypo-fractionated stereotactic radiotherapy. Radiat Oncol. 2016;11:92.

[3] Yano M, Yoshida J, Koike T, Kameyama K, Shimamoto A, Nishio W, Yoshimoto K, Utsumi T, Matsumura Y, Moriyama S, Fujii Y. The outcomes of a limited resection for non small cell lung cancer based on differences in pathology. World J Surg. 2016. doi: 10.1007/soo268-016-3596-9.

[4] Wu CC, Maher MM, Shepard JA. Complications of CT-guided percutaneous needle biopsy of the chest: prevention and management. AJR Am J Roentgenol. 2011;196(6):W678-W682.I

[5] Ravindra Patil et.al An Approach Toward Automatic Classification of Tumor Histopathology of Non-Small Cell. Tomography Jr.

[6] Gillies R, Kinahan P, Hricak H (2016) Radiomics: images are more than pictures, they are data. Radiology 278:2

[7] Lambin P, Rios-Velazquez E, Leijenaar R et al (2012) Radiomics: extracting more information from medical images using advanced feature analysis. Eur J Cancer 48:441-446.

[8] Kumar V, Gu Y, Basu S et al (2012) Radiomics: the process and the challenges. Magn Resonance Imaging 30(9):1234-1248

[9] Aerts HJ, Velazquez ER, Leijenaar RT et al (2014) Decoding tumour phenotype by noninvasive imaging using a quantitative Radiomics approach. Nat Commun 5:40

[10] Mandelbrot, B. B. The Fractal Geometry of Nature. New York: W. H. Freeman, 1982.

[11] James W. Baish and Rakesh K. Jain, Fractals and Cancer, Perspectives in Cancer Research, 2000

[12] Fractals: a possible new path to diagnose and cure cancer?, Future Oncol. (2015) 11(22), 304930513.

[13] Heymans, O., Blacher, S., Brouers, F., and Pierard, G. E. Fractal quantification of microvasculature heterogeneity in cutaneous melanoma. Dermatology (Basel), 198: 212-217, 1999.

[14] D. Cusumano et.al Development and Validation of New Radiomic Features Based on Fractal Analysis. International Journal of Radiation Oncology 2017.

[15] https://www.wahl.org/fe/HTML_version/link/FE4W/c4.htm, last accessed and verified on 4th Nov 2018

[16] Automated chest X-ray screening: Can lung region symmetry help detect pulmonary abnormalities?. IEEE Transactions on Medical Imaging. 2018

[17] Feature Selection for Automatic Tuberculosis Screening in Frontal Chest Radiographs, Vajda, S., Karargyris, A., Jaeger, S. et al. J Med Syst (2018) 42: 146. https://doi.org/10.1007/s10916-o180991-9

[18] Foreign Circular Element Detection in Chest X-rays for Effective Automated Pulmonary Abnormality Screening. International Journal of Computer Vision and Image Processing(IJCVIP), IGI Global

[19] Edge map analysis in Chest X-rays for Automatic Abnormality Screening, International Journal of Computer Assisted Radiology \& Surgey (IJCARS), Springer

[20] Szigeti, Szabó , Korom, Czibak, Horváth. Radiomics-based differentiation of lung disease models generated by polluted air based on X-ray computed tomography data. BMC Medical Imaging, Vol 16, Issue 1, Pages 14. February 11, 2016.https://doi.org/10.1186/s1288o-o16-o118-z

[21] David Cusumano, Nicola Dinapoli, Luca Boldrini, Giuditta Chiloiro et al. Fractal-based radiomic approach to predict complete pathological response after chemo-radiotherapy in rectal cancer. 
La radiologia medica, 2017.

[22] Juszczak, P.; D. M. J. Tax; R. P. W. Dui. Feature scaling in support vector data descriptions. Proc. 8th Annu. Conf. Adv. School Comput. Imaging: 25-30. 2002 


\section{Chapter 8}

End-to-End Approach to Build Diagnostic Radiomics models

Prasad R V, Ravindra Patil, Ashish Jha , Sneha Mithun, Venkatesh Rangarajan , Leonard Wee and Andre Dekker

Using Artificial Intelligence and Radiomics to support cancer care decisions, 19th International Conference on the use of Computers in Radiation Therapy, 2019, 


\begin{abstract}
This work describes a framework for leveraging the cloud platform and big data technologies to perform medical image data analytics across multiple institutions. The key challenges the authors have addressed are the security, privacy requirements, scalability of the solution and use of cloud based analytics for the existing proprietary legacy systems with network connectivity in hospitals. The Digital Imaging and Communication in Medicine (DICOM) medical images acquired for clinical purposes are to be shared with radiologists and institutions for interpretation of images to support disease diagnosis, who are miles away from the institutions. Prior to sharing DICOM images with third parties, the authors need to follow strict guidelines with respect to de-identification of Protected Health Information (PHI) from the medical data to be shared. To de-identify PHI information from images the authors have devised a newer generation de-identification techniques based on the big data platform to obscure privacy information from DICOM tags, embedded text in images and face information from CT and MR images.
\end{abstract}

Further to facilitate accurate analysis on medical images, a Clinical Decision Support System (CDSS) inference model is developed. In this work, the authors proposed use cases and developed a cloud-based framework to distribute/share clinical images with a CDSS for radiologists on the move and to the institutions that do not have an on-premise solutions. The developed solution satisfies all the PHI de-identification requirements and supports scalability by efficiently using big data cloud technologies, for faster and reliable processing. As a demonstration, a survival prediction model application is built using this approach.

Keywords : De-identification, picture archiving and communication system, medical image analytics, big data platform, deep learning in cloud 


\section{Introduction}

A challenge of any busy cancer hospital is the increasing burden of the number of patients who need to be provided with a consistent and "first time right" diagnosis and treatment plan that uses the hospital resources - equipment and human experts - optimally [1]. An IT Infrastructure that involves artificial intelligence based predictive models that can predict outcomes might be helpful in overcoming above-mentioned challenges [2]. These outcome prediction models might make use of Radiomics features, which can be extracted from tumors delineated by physicians Besides, the workflow is optimized further by integrating an automatic tumor segmentation algorithm with high performance accuracy to deliver faster care to patients.

Several Clinical Decision Support (CDS) modules have been built to address the health care challenges [3-9]. However they lack seamless integration with the existing IT infrastructure of a hospital or its use by the healthcare provider if it deviates the existing workflow of the operation [10]. In addition, another major hurdle for data accessibility is the fragmented and diverse non-interoperable systems within a hospital and across institutions. There is a lack of standards and there are many proprietary systems, which make data sharing almost an impossible task. Hence, there is a need to build the solutions that can co-exist with the current IT infrastructure of the hospital as well as augment the existing workflow, with minimal deviation to the existing process and minimal human intervention. In this work, we built an End to End CDS pipeline involving image analytics to work in the hospital environment.

\section{Materials \& Methods}

The proposed solution is a modular software platform deployed in a busy cancer hospital in India consisting of modules for advanced visualization and highly automated processing of images, including:

- Retrieving the images from the PACS and anonymizing the data set

- Automatic segmentation of tumors and export as DICOM RT Structure Sets (RTSTRUCT)

138 | Prognostic and Prediction Modelling with Radiomics for Non-Small Cell Lung Cancer 
- Automatic computation of Radiomics features

- Conversion of Radiomics features to FAIR (Findable Accessible, Interoperable, Reusable) data using ontologies and Semantic Web technology [11]

- Integrating the non-imaging clinical patient information and overlaying it with the image information using Semantic Web technology

\section{Retrieving of images from PACS and anonymizing}

Data sharing is increasingly recognized as critical to cross-disciplinary research and to assure scientific validity. Despite National Institutes of Health and National Science Foundation policies encouraging data sharing by grantees, little sharing of clinical data has in fact occurred. A principal reason often given is the potential of inadvertent violation of the Health Insurance Portability and Accountability Act privacy regulations or similar regulations in other jurisdictions [12]. While regulations specify the components of private health information that should be protected, there are no commonly accepted methods to de-identify clinical data objects such as images. This leads institutions to take conservative risk-averse positions on data sharing. In imaging trials, where images are coded according to the DICOM standard, the complexity of the data objects and the flexibility of the DICOM standard have made it especially difficult to meet privacy protection objectives. The recent release of DICOM Supplement 142 on image de-identification has removed much of this impediment [13].

The field of header tag de-identification is a well-matured one and many third party tools provide support for anonymization of DICOM tags. A number of toolkits exist for DICOM header tags de-identification. A comprehensive list of DICOM anonymizers along with their download locations is provided at [3]. A recent study comparing ten DICOM anonymizer tools is reported in [4]. In our approach we built a custom DICOM De Identification module to mask all the tags that contains the PHI information of the subject so that the privacy of the subject is preserved. It also well known that all legacy PACS have been on premise and closed solutions. The issue with this approach is that the image sharing across health care enterprises is almost impossible [14]. However, with cloud technology advancements and de-identification modules being embedded and widely used, it is imperative to move the PACS also to the cloud and many 
PACS vendors have already forayed into that path. Nevertheless, most of them either provide cloud-only or premise-only models. However, the need of the hour is to develop a hybrid model [15] where the solution works both on-premise and can extend to a cloud model on-demand either for archival or to access by health care professionals and data scientists. In our approach, we extracted the data from on-premise data and pushed the data to a computation node after performing de-identification for further processing.

\section{Automatic tumor segmentation export as DICOM RT Structure Sets (RTSTRUCT)}

Machine learning and deep learning technologies are gaining popularity in medical image analytics due to the availability of big data platforms. A clinical decision support system based on the image analytics on big data, might lead to improved productivity and accuracy of diagnoses by radiologists and clinicians. In particular, for radiotherapy planning, a significant amount of time is spent on contouring the region of interest in particular the Gross Tumor Volume (GTV). In this work, we present a Deep Learning (DL) model to demonstrate the application of the framework. We designed a system using a U-net deep neural network to automatically delineate the GTV in CT Lung studies (described in Chapter 4). Architectural details of the system are given in figure 1. The challenges of segmenting a part of the lung as a 'Region of Interest' in CT images and delineating the GTV, is complicated by inter-observer variability, object variability, image quality and varying tumor sizes and shapes. We used the platform to detect a GTV using the deep learning model. Further, these delineated GTV's were stored as RTSTRUCT for further analysis. 


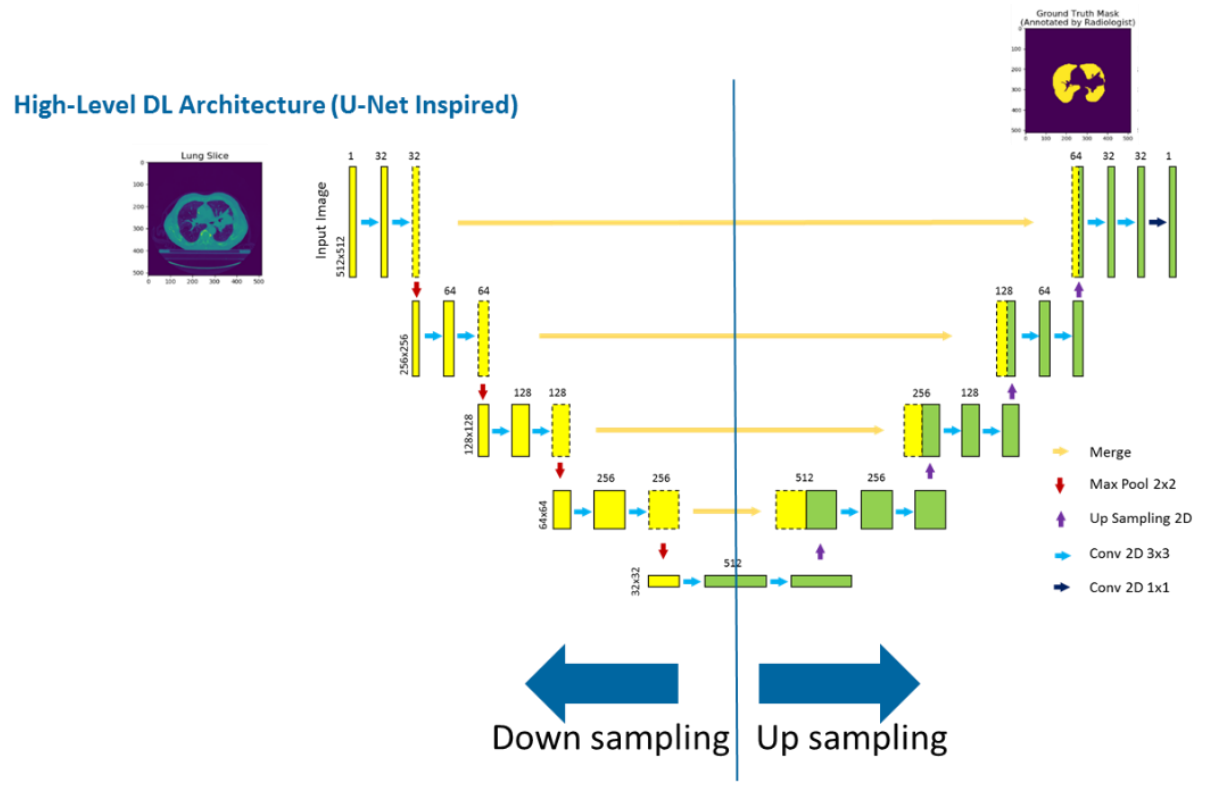

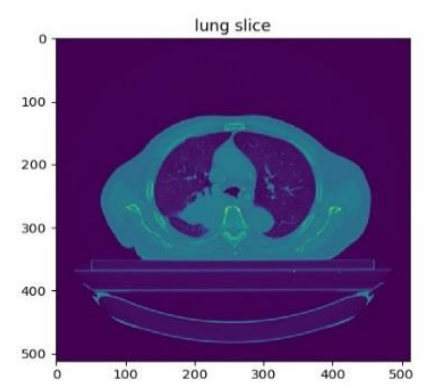

Lung Slice

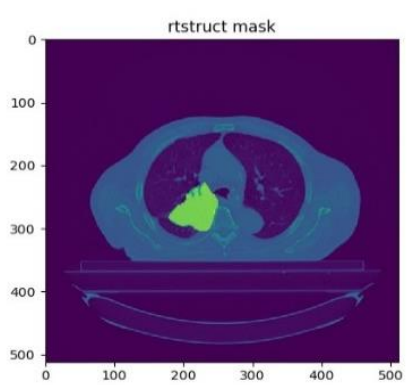

Ground Truth

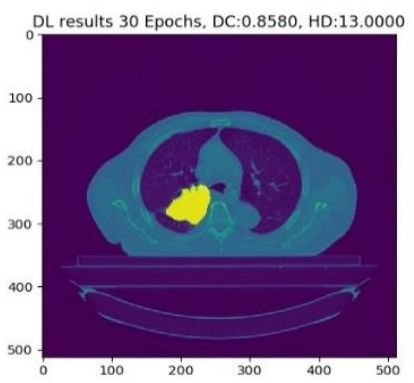

Model Output

Figure 1. DL model architecture and Gross Tumor Volume results

\section{Automatic computation of the Radiomics features}

There is a huge amount of data captured during medical imaging. Radiologists study the images in depth and annotate the relevant portions of an image set for diagnostic reporting. The most common features used are the count, location, size and volume of the findings. However, these features are not sufficient for building a personalized predictive model. Radiomics is a fast advancing medical field, which aims to extract more information from the images directly with algorithms, for clinical decision making [16]. In this experiment from the delineated GTV from the above steps approximately $\sim 1000$ Radiomics features were extracted ranging from first order features such as Energy, Entropy, Kurtosis, Maximum, Mean etc. to 
wavelet based features, GLCM and GLRM features along with different shape and size features. Furthermore, these features are stored as "triples" (see below), so that these features can be queried through a SPARQL end point for easy access and use of these features to build CDS solutions.

\section{Conversion of Radiomics Features to FAIR}

Although medical informatics and diagnostics technologies are well established in hospitals, the data generated by diagnostic technologies is not yet fully exploited to improve hospital workflow, operations and patient outcomes. One of the reasons being that the data is not completely Findable, Accessible, Interoperable and Re-usable (FAIR) [17]. FAIR data is a key enabler for a successful implementation of a learning system. Widespread adoption of the FAIR principles in storing medical data would increase the availability of data for learning systems [18]. Semantic Web technologies such as RDF (Resource Description Framework) are standardized by the World Wide Web Consortium $\left(\mathrm{W}_{3} \mathrm{C}\right)$, for sharing data and meaning by machines and beyond boundaries [19]. The RDF provides a simple mechanism to link the subject and predicate by a relation, known as a triple. This description is made unique by means of a Unique Resource Identifier (URI) [20]. In our current approach all the Radiomics features extracted from the GTV were stored as RDF for easy accessibility and also for performing distributed computing thereof.

\section{Integrating the non-imaging Clinical patient information}

Features derived from the image, using image analytics are important in building CDS algorithms. However, the performance of algorithms can generally be improved by using nonimaging information such as a patient'sage, gender and the clinical history. It is imperative to have all the imaging features stored along with non-imaging features , such that these features can be combined in improving the predictive power of the CDS algorithm. In this work, authors integrated the non-imaging clinical patient information with that of imaging features, stored both as triples and used them to build a survival model for NSCLC subjects. The detailed 
approach of building the CDS by combining both feature domains has been explained in Chapter 5 of this thesis.

\section{Orchestration}

The above steps were integrated into the existing radiology and clinical workflow to develop a model to predict a cancer outcome such as survival (Chapter 5). Figure 2 shows the high level overview of the workflow and a schematic representation of the integrated solution.

The solution consists of a modular approach, where multiple independent components can be used as plug and play. Modules include a DICOM node to process the imaging information coming from the scanners such as CT and MR. Further modules process these images and send them across to the PACS as well as to a Plugin and Algorithm Infrastructure (PAI). The PAI consist of many modules that aid in automating the model building approach. It consists of an Image Processing module, which can perform preprocessing operations such image noise correction, motion artifact detection and filtering. The Workflow Orchestrator is a specific rule engine that aids in designing the workflow based on the image type and the algorithms that need to run on these images to achieve the desired results. The PIA also contains different AI modules such as a module for Radiomics extraction, auto tumor segmentation and developing survival models. All these AI modules are built using container technology (Docker) and can run independently based on a defined input. The Docker Configurator helps in orchestrating the different AI algorithm dockers. In addition, PIA consist of data converter plugins, to store the intermediate algorithm output such as DICOM2RDF. In addition, to visualize the results an image viewer module is integrated into the PIA such that a clinician can analyze the segmentation and other algorithms output for analysis.

The proposed workflow for learning a survival model, consist of

- A DICOM node

- A deep learning based GTV auto-segmentation module

- A viewer module to give the radiologist the ability to review the tumor segmentation and make changes to the contour wherever needed. 
- A Radiomics docker to extract the Radiomics features and these features are stored as RDF.

- A survival model learning module where the Radiomics features are combined with the subject metadata.

The proposed architecture takes into account the ability to tailor the workflow to address different cancers and outcomes and to fine-tune the workflow for a particular cancer wherein an additional AI algorithm needs to be included in the workflow. This makes the solution disease-agnostic, scalable and rapidly customizable.

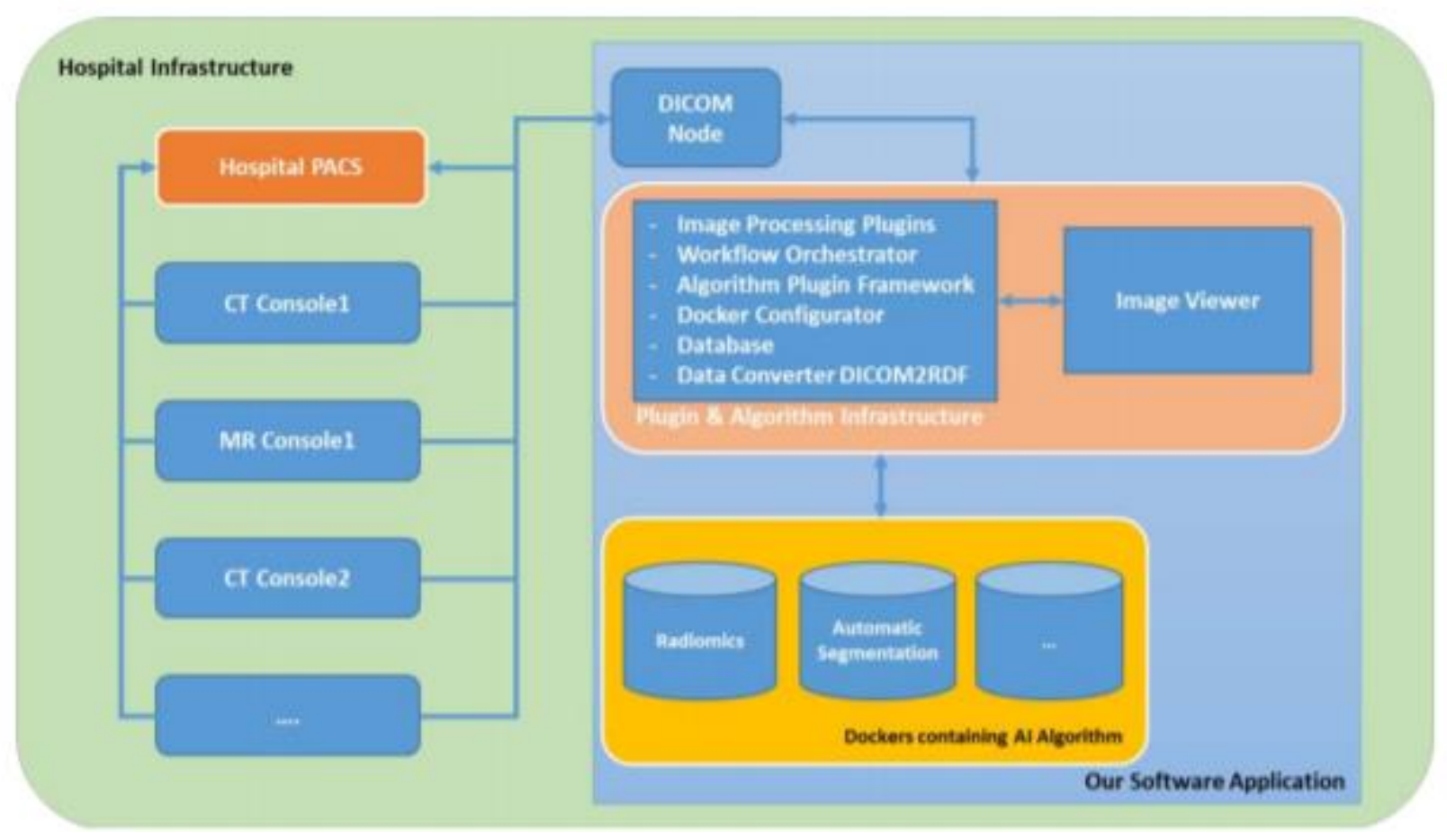

Figure 2. High Level Overview

\section{Conclusion}

By making the features FAIR, the results can be shared remotely and queried using Semantic Web standards such as SPARQL. Sharing helps in building models to predict clinical outcomes that are relatively uncommon events, such as recurrences and treatment-related toxicities, as this requires a large numbers of cases to be analyzed together in order to yield 
statistical significance. The proposed solution, with an integrated automation and smart workflow orchestration, is extremely useful in reducing the time spent by clinicians on processing of image data and data conversion to make it compatible for AI purposes. This entire workflow is currently demonstrated for CT based images for non-small cell lung cancer (NSCLC). In future work, this framework could be used to integrate automatic segmentation algorithms specifically for cervical cancer, head and neck cancer and rectal cancer as these are the most lethal cancers within developing countries. 


\section{References}

[1] Massat MB. Precision Imaging: An important component of precision health care. Appl Radiol. 2019;8(4):44-47.

[2] Islam MS, Hasan MM, Wang X, Germack HD, Noor-E-Alam M. A Systematic Review on Healthcare Analytics: Application and Theoretical Perspective of Data Mining. Healthcare (Basel). 2018 May 23;6(2):54. doi: 10.3390/healthcare6020054. PMID: 29882866; PMCID: PMC6023432.

[3] Lambin P, van Stiphout RGPM, Starmans MHW, Rios-Velazquez E, Nalbantov G, Aerts HJWL, et al. Predicting outcomes in radiation oncology--multifactorial decision support systems. Nat Rev Clin Oncol. 2013 Jan;10(1):27-40.

[4] Valdes G, Simone CB, Chen J, Lin A, Yom SS, Pattison AJ, et al. Clinical decision support of radiotherapy treatment planning: A data-driven machine learning strategy for patient-specific dosimetric decision making. Radiother Oncol J Eur Soc Ther Radiol Oncol. 2017;125(3):392-7.

[5] Hemens BJ, Holbrook A, Tonkin M, Mackay JA, Weise-Kelly L, Navarro T, et al. Computerized clinical decision support systems for drug prescribing and management: A decision-makerresearcher partnership systematic review. Implement Sci. 2011 Aug 3;6(1):89.

[6] Nieuwlaat R, Connolly SJ, Mackay JA, Weise-Kelly L, Navarro T, Wilczynski NL, et al. Computerized clinical decision support systems for therapeutic drug monitoring and dosing: A decision-maker-researcher partnership systematic review. Implement Sci. 2011 Aug 3;6(1):90.

[7] Souza NM, Sebaldt RJ, Mackay JA, Prorok JC, Weise-Kelly L, Navarro T, et al. Computerized clinical decision support systems for primary preventive care: A decision-maker-researcher partnership systematic review of effects on process of care and patient outcomes. Implement Sci. 2011 Aug 3;6(1):87.

[8] Roshanov PS, Misra S, Gerstein HC, Garg AX, Sebaldt RJ, Mackay JA, et al. Computerized clinical decision support systems for chronic disease management: A decision-maker-researcher partnership systematic review. Implement Sci. 2011 Aug 3;6(1):92.

[9] Lustberg T, van Soest J, Gooding M, Peressutti D, Aljabar P, van der Stoep J, et al. Clinical evaluation of atlas and deep learning based automatic contouring for lung cancer. Radiother Oncol. 2018 Feb 1;126(2):312-7

[10] Ash JS, Sittig DF, Campbell EM, Guappone KP, Dykstra RH. Some unintended consequences of clinical decision support systems. AMIA Annu Symp Proc AMIA Symp. 2007 Oct 11;26-30.

[11] Wilkinson MD, Verborgh R, Bonino da Silva Santos LO, Clark T, Swertz MA, Kelpin FDL, Gray AJG, Schultes EA, van Mulligen EM, Ciccarese P, Kuzniar A, Gavai A, Thompson M, Kaliyaperumal R, Bolleman JT, Dumontier M. 2017. Interoperability and FAIRness through a novel combination of Web technologies. PeerJ Computer Science 3:e110 https://doi.org/10.7717/peerj-cs.110

[12] John B. Freymann et. Al, "Image Data Sharing for Biomedical Research-Meeting HIPAA Requirements for De-identification” J Digit Imaging (2012) 25:14-24.

[13] David Clunie, De-identification Revisited DICOM Supplement 142, DICOM INTERNATIONAL CONFERENCE \& SEMINAR, Oct 9-11, 2010 Rio de Janeiro, Brazil.

[14] Cloud PACS: http://appliedradiology.com/articles/roadmap-to-cloud-based-pacs, Last Accessed date 26 July 2019.

[15] Hybrid Models: https://www.itnonline.com/article/what-vendor-neutral-anyway, Last Accessed Date 26 July 2017.

[16] Hugo J. W. L. Aerts, Emmanuel Rios Velazquez, Ralph T. H. Leijenaar, Chintan Parmar, Patrick Grossmann, Sara Carvalho, Johan Bussink, René Monshouwer, Benjamin Haibe-Kains, Derek Rietveld, Frank Hoebers, Michelle M. Rietbergen, C. René Leemans, Andre Dekker, John Quackenbush, Robert J. Gillies \& Philippe Lambin. Decoding tumour phenotype by noninvasive imaging using a quantitative Radiomics approach. Nature Communications 5, Article number: 4006 (2014) doi:10.1038/ncomms50o6.

[17] Go FAIR Initiative : https://www.go-fair.org/fair-principles/

146 | Prognostic and Prediction Modelling with Radiomics for Non-Small Cell Lung Cancer 
[18] Kubben Pieter, Dumontier Michel, Dekker Andre, Fundamentals of Clinical Data Science, Springer Open, 2019. ISBN 978-3-319-99712-4 ISBN 978-3-319-99713-1 (eBook) https://doi.org/10.1007/978-3-319-99713-1

[19] https://www.w3.org/standards/semanticweb/

[20] Clark T. (2013) Uniform Resource Identifier (URI). In: Dubitzky W., Wolkenhauer O., Cho KH., Yokota H. (eds) Encyclopedia of Systems Biology. Springer, New York, NY 


\section{Chapter 9}

Discussion and Future Perspectives

Ravindra Patil

148 | Prognostic and Prediction Modelling with Radiomics for Non-Small Cell Lung Cancer 
In this chapter, a discussion of the key findings and lessons learned is provided. Also, suggested are directions for future research and exploration.

During this thesis, we have touched upon the multiple challenges in the area of predictive model development for NSCLC utilizing a Radiomics approach. Our excursion has taken us into deep learning neural networks for the purpose of automating the tumour ROI segmentations that are essential for Radiomics analysis, and we have also looked at the significance of fractional dimension as a type of morphological Radiomics feature. In the previous chapter, we turned our attention to a proposal for integrating these models into a healthcare Radiomics platform so as to reduce the data handling burden on the treating clinician.

A key overall finding throughout this thesis work is the need to efficiently build prognostic and predictive models, including robust validation, so these models could provide relevant information to clinicians at the right time to make appropriate decisions. There are multiple prediction models that exists in cancer care; however, their use has not been fully exploited to date due to multiple challenges in deployment of these algorithms in real-life clinical settings. One of the challenges is that the models needs to become more generalizable to different populations. In addition, validated models need to be able to adapt seamlessly into and be readily integrated with an existing clinical workflow. These issues were discussed in Chapter 2 and some recommendations were provided on how high quality prediction models can be built.

Another overall finding was the need for better automation of the workflow without mundane manual intervention, so that these prognostic algorithms can be more easily developed and then deployed into clinical use. This approach has been addressed by developing deep learning based segmentation models for lung and GTV so that the ROI is automatically delineated without any seed point initialization. This approach would help to significantly reduce the time spent in performing manual contouring and also aids in decreasing inter and intra operator 
variability of the delineation results - an issue often encountered in physician based delineations. The results of automatic delineation were discussed in Chapter 3 and 4 .

There remains, however, further scope to improve these auto segmentation models. The current Deep Learning models are little unstable when images of different format such as full body scans being passed for segmentation and with noise. However, the advantage of being able to re-train AI on progressively more diverse data makes it gradually more robust over time. This should ideally lead to more reliable segmentations and reduce false positives.

Radiomics offers immense possibilities to increase throughput though quantitative mining of features and provide key information to support a clinician's decision-making such as survival analysis. It is quite important that the prognosis of the disease be correctly explained to the patient, so that the patient can plan his/her activities in a timely fashion. One of the approach is to predict the personalized survival time of the patient. For this, we built in chapter 5 a survival model that used Radiomics features and demonstrated that the model with a combination of image-derived Radiomics features combined with clinical data of the patient provides a better prediction of survival than the existing approaches, which only consider the clinical data. A possible future extension of work is to improvise the concordance index by adapting automated feature selection approaches. In addition, there is a need of other important outcomes that are not addressed in this thesis such as recurrence, disease-specific survival, treatment-related toxicities that are important for both clinicians and patients for their decision making and planning. However, obtaining these longitudinal data to build the models for these outcome might be challenging.

Identification of the histology of NSCLC is important for treatment planning. Ideally, biopsy is the preferred method, which is invasive in nature and has side effects. In one of the experiments in chapter 6 , we performed auto classification of the NSCLC histology using Radiomics features, thereby paving the way for an alternate approach to biopsies using imaging, which is noninvasive and has potential to be considered as a virtual biopsy. In the near future, with better models, it is quite possible to start considering virtual biopsy as another approach for treatment planning. As the recent data with the cytotoxic drug pemetrexed suggested that histology could also influence outcome in NSCLC patients undergoing chemotherapy.

150 | Prognostic and Prediction Modelling with Radiomics for Non-Small Cell Lung Cancer 
Similarly, in Chapter 7 we describe a methodology to derive a new feature called Fractal dimension to capture heterogeneity of the tumor based on the tumor contour and shape. This feature was an additional contribution to the existing radiomic features. In this study, it was concluded that the Fractals based features play an important role in the histology classification of tumors. However, there exist still an opportunity to improvise the current work on the fractal based approach by considering 3-D volume of the data, to better capture the heterogeneity.

The primary requirement for data mining and Radiomics is to enable the hospital throughput and support decision-making. The challenges of successfully deploying a CDSS in the hospital is elaborated and discussed in detail in Chapter 9, concluding that automated approaches tailored towards existing hospital IT infrastructure along with good governance and deployment strategies for data and AI models in the hospitals, is a must for the effectiveness of clinical decision support systems.

\section{Future Perspectives}

In this section, an outlook is given on the challenges and future work in the area of technologies related to auto segmentation, Radiomics based clinical models and deployment of AI based CDSS.

\section{Segmentation of Anatomy or Morphology of Interest}

Segmentation is an active field of current research in healthcare, as identification of the region of interest accurately without time-consuming manual effort is needed for building accurate and reliable clinical decision support systems. To find better segmentation accuracy, multiple models were developed ranging from simple threshold to that of feature based machine learning models. However, with the advent of Deep Learning algorithms these computer vision problems have been addressed with renewed focus yielding better accuracy than traditional Prognostic and Prediction Modelling with Radiomics for Non-Small Cell Lung Cancer | 151 
approaches. In particular, this is true for healthcare related segmentation algorithms built using deep learning. In our work too, we developed deep learning based segmentation algorithms to segment region of lung as well as the Gross tumor volume in diseased lung conditions. Though the results are promising, there are still improvements that can be carried out.

In particular, the current models were trained only on the chest CT images, and there is quite a possibility that if a full body scan is passed to these algorithms, that the algorithm can start detecting false positives. In addition, this study was conducted on $2 \mathrm{D}$ contour GTV regions and may be extended to $3 \mathrm{D}$ using mesh approaches increasing the detection accuracy. Furthermore, the automatic detection of the GTV can be extended to provide even the Planning Target Volume (PTV) and Clinical Target Volume (CTV). Improving the accuracy and building intelligent multi organ segmentation models without manual intervention for any disease condition is also still an unmet need.

Another area of research focus that is interesting and relevant is building self-explainable AI models, as current AI models, in particular deep learning models, might be good at performing the said task such as segmentation, but they fail to explain why a particular ROI such as the GTV was segmented. Adding the explainability to the model output would help in building confidence of the healthcare provider in the model so that the trust on algorithms can be established. One of the approaches that we adapted in our work in Chapter 3 is to bring explainability to the model by incorporating the grad-cam visualization so that neural activations are mapped back to the spatial location of the image, to ascertain what region in the given image caused a particular activation. However, still there is potential to improve the explainability context by adding the explanation, which can be understood more commonly such as, "the following tumor region was segmented because the region had following intensity variations, heterogeneity and the shape...”. This could be achieved by combining Natural Language Processing with that of grad-cam visualization, which is more intuitive so that a better explanation can be achieved. 


\section{Building Prognostic Models}

Radiomics is currently used for developing algorithms to predict overall survival, other tumor outcomes such as distant control and for identifying the histology and classification of the nodules etc. It has shown a promising trend and potential in aiding both diagnosis and prognosis of NSCLC with the main challenges being reproducibility. The validation and generalization of these models remains a significant challenge, as most of the models are built on small data and are not validated on an external dataset or validated on limited internal data from the same institute leading to a lack of generalizability. In addition, the vast majority of studies did not share their training or test data, although utilities such as TCIA archive (https://www.cancerimagingarchive.net/) and BMIA (https://xnat.bmia.nl/) exist for such sharing and do have well annotated image datasets from large studies including in lung cancer.

In addition, investigations of feature repeatability and reproducibility are currently limited to a small number of cancer types. Reporting quality could be improved in regards to details of: feature extraction software, digital image manipulation (pre-processing) and cut-off value used to distinguish stable features. This was discussed in Chapter 2 of this thesis and it was suggested that authors publish image collections (including delineations) and feature extraction software as open access resources, to accelerate progress in clinical generalizability and independent validation of models. Also, the fractals study was limited to CT images, this may be extended to MR images and combined with other tissue characteristics derived from a MR image, to improve diagnosis and treatment decisions.

The challenge of data paucity a researcher face in healthcare to build data driven models is well known. In order to overcome this challenge the concept of distributed learning (also known as federated machine learning) can be employed and is gaining traction worldwide. In this approach, a local model learns on a local database, the local models then collaborate in a distributed platform to propose a global model based on exchanging model weights/gradient (which can be performed without exchanging individual patient data) and transfer learning approaches. This global model is validated on the local databases of the participating sites. This concept is being experimented by the 'Big Imaging data for Oncology in Netherlands and India Prognostic and Prediction Modelling with Radiomics for Non-Small Cell Lung Cancer | 153 
Collaboration' (BIONIC) project. The objective of the BIONIC project is to develop technology that allows global distributed learning and local deployment of clinical decision support systems (DSSs) based on Big Imaging Data analytics.

\section{CDSS deployment}

There is tremendous growth in how AI is being adapted in healthcare and in particular in cancer care. The use cases of AI models that are being prototyped range from developing segmentation models, auto delineation of organs at risk to that of determining the histology using noninvasive approach. In addition, there are multiple applications that have been developed to improve the cancer workflow, by integrating AI tools for auto scheduling and detecting cancer stage to that of work list prioritization based on the severity of the subject condition. In particular, the use of Deep Learning has shown enormous potential, however the access to large and high quality data to train these models remains a challenge. The need of the hour, is efficient and effective data collection and curation methods and solutions. Also, to build AI solutions that can fit into the existing workflow of the clinicians, without much impact. At the same time, clinicians and end users are bogged down by the extensive data entry and reporting tasks. Startups and technology providers are experimenting with various tools to resolve the clinician's dichotomy. A few technology examples are electronic writing paper, speech recognition software supported by medical ontologies and intelligent bots to capture the information.

Technology advances are rapidly changing the workflow and day-to-day operations in a hospital. The FDA guidelines and regulations are evolving to keep pace with the artificial intelligence based software providing clinical decision support. As elaborated in Chapter 8, hospitals need to be better equipped with the right processes and measures to commission and deploy AI based decision support software.

\section{Concluding Remarks}

Tremendous growth in the imaging data and the requirement for having a personalized cancer care to provide better outcome, with improved patient and staff satisfaction is need of the 154 | Prognostic and Prediction Modelling with Radiomics for Non-Small Cell Lung Cancer 
healthcare industry. This thesis was on attempt to address a small selection of the aforementioned aspects. The work provides a proof of concept that virtual biopsy, considering a Radiomics approach, can be used to identify different histologies of NSCLC. To overcome the challenges of segmentation of ROIs that are needed in Radiomics analysis, the approach of Deep Learning was described in this work, that has a potential to alleviate some burden of manual delineation from the oncologist, reduce inter/intra-observer segmentation variations and also help in building the confidence of oncologist on these models by incorporating some degree of AI explainability. Fractal dimension has been introduced as a potential new class of informative Radiomics feature. The survival models developed by considering both Radiomics and clinical features showed promising improvement over traditional clinical features-only based model. A comprehensive and quality-enhancing proposal for deployment of Radiomics models as clinical decision support in real-world hospital environments is needed to exploit this emerging software technology. In conclusion, the work done in this thesis indicates the role of AI models aiding in improving cancer care in particular NSCLC, thereby improving the quality of care that can be offered to the patients. 


\section{Chapter 10}

Summary

Ravindra Patil

156 | Prognostic and Prediction Modelling with Radiomics for Non-Small Cell Lung Cancer 
The improvement in cancer care through automation and personalization is required to provide cost effectiveness, improved patient and staff experience and better health outcomes. With the advancement in AI there is a strong potential to achieve these improvements. In this thesis, the approach adapted in building predictive models for NSCLC and the usage of machine learning and deep learning models to enhance the accuracy and automation of endto-end clinical decision support systems that aims at improving the cancer care was explored.

The thesis began with general introduction of the Radiomics and associated challenges with workflow and application in Chapter 1. There is strong need to build high quality prognostics and predictive models in cancer care, so that these can be rapidly adapted into the clinical practice. Chapter 2 provides details on different methodologies that are used for modelling with Radiomics, the associated challenges and recommendation on how to build high quality prognostics and predictive models.

With the need to have automation in the cancer care, to reduce the burden on oncologist and reduce inter/intra-observer segmentation variations, Chapter 3 was introduced to describe Deep Learning based approach to segment the region of lung, without seed point initialization. In addition, the chapter highlighted the effectiveness of Deep Learning over traditional segmentation approaches. In continuation with the thirst of automation, Chapter 4 described an approach of auto extraction of the Gross Tumor Volume from CT slices using Deep Learning, there by demonstrating significant reduction of time compared to manual contouring.

It is quite important that the prognosis of the disease be correctly explained to the patient, so that the patient can plan his/her activities in a timely fashion. For this, we built in Chapter 5 a survival model that used Radiomics features and demonstrated that the model with a combination of image-derived Radiomics features combined with clinical data of the patient provides a better prediction of survival than the existing approaches, which only consider the clinical data.

Identification of the histology of NSCLC is important for treatment planning. Ideally, biopsy is the preferred method, which is invasive in nature and has side effects. In Chapter 6, experiment was conducted to auto classify NSCLC histology using Radiomics features, thereby paving the way for an alternate approach to biopsies using imaging, which is noninvasive and has potential to be considered as a virtual biopsy. In Chapter 7 , the Radiomics techniques were further extended by introducing fractals as new features, which aids in quantification of Prognostic and Prediction Modelling with Radiomics for Non-Small Cell Lung Cancer | 157 
irregular patterns and demonstrated the role of fractals in improving histology classification of NSCLC.

Finally, in Chapter 8, system level needs and techniques to build End to End diagnostic Radiomics model for clinical application was explored, along with good governance and deployment strategies for data and AI models in the hospitals were discussed. In conclusion, Chapter 9 provides a general discussion of the results presented in this thesis and related future perspectives in the field of automation in healthcare. 


\section{SAMENVATTING}

De verbetering van de kankerzorg door automatisering en personalisatie is vereist om kosteneffectiviteit, verbeterde patiënt- en personeelservaring en betere gezondheidsresultaten te bieden. Met de vooruitgang in AI is er een groot potentieel om deze verbeteringen te bereiken. In dit proefschrift is de aanpak aangepast bij het bouwen van de voorspellende modellen voor NSCLC en het gebruik van machine learning en deep learning-modellen om de nauwkeurigheid en automatisering te verbeteren van het bouwen van end-to-end klinische beslissingsondersteunende systemen die gericht zijn op het verbeteren van de kankerzorg en in het bijzonder voor NSCLC werd onderzocht.

Het proefschrift begon met de algemene introductie van de Radiomics en de bijbehorende uitdagingen met workflow en toepassing in hoofdstuk 1. Er is sterke behoefte aan het bouwen van hoogwaardige prognostica en voorspellende modellen in de kankerzorg, zodat deze snel kunnen worden aangepast aan de klinische praktijk. Hoofdstuk 2 geeft details over verschillende methodologieën die worden gebruikt voor modellering met Radiomics, de bijbehorende uitdagingen en aanbevelingen voor het bouwen van hoogwaardige prognostica en voorspellende modellen. Omdat er behoefte is aan automatisering in de kankerzorgcyclus om de last voor de oncoloog te verminderen en de variaties tussen segmenten binnen en tussen de waarnemers te verminderen, werd hoofdstuk 3 geïntroduceerd om een op Deep Learning gebaseerde benadering te beschrijven om het longgebied te segmenteren, zonder initialisatie van het startpunt. Daarnaast belichtte het hoofdstuk de effectiviteit van Deep Learning ten opzichte van traditionele segmentatiebenaderingen. In vervolg op de dorst naar automatisering, beschreef Hoofdstuk 4 een benadering van automatische extractie van het Bruto Tumor-volume uit CT-segmenten met behulp van Deep Learning, daar door een significante vermindering van de tijd te demonstreren in vergelijking met handmatige contouring.

Het is heel belangrijk dat de prognose van de ziekte correct wordt uitgelegd aan de patiënt, zodat de patiënt zijn / haar activiteiten tijdig kan plannen. Hiervoor hebben we in Hoofdstuk 5 een overlevingsmodel gebouwd dat gebruik maakte van Radiomics-kenmerken en aantoonde dat het model met een combinatie van beeldgerelateerde Radiomics-kenmerken 
gecombineerd met klinische gegevens van de patiënt een betere voorspelling van overleving biedt dan de bestaande benaderingen, die alleen rekening houden met de klinische gegevens. Identificatie van de histologie van NSCLC is belangrijk voor behandelplanning. Idealiter is biopsie de voorkeursmethode, die invasief van aard is en bijwerkingen heeft. In Hoofdstuk 6 werd experiment uitgevoerd om NSCLC-histologie automatisch te classificeren met behulp van Radiomics-functies, waardoor de weg werd vrijgemaakt voor een alternatieve benadering van biopsieën met behulp van beeldvorming, die niet-invasief is en potentieel kan worden beschouwd als een virtuele biopsie. In Hoofdstuk 7 werden de Radiomics-technieken verder uitgebreid door fractals als nieuwe kenmerken te introduceren, die helpen bij het kwantificeren van onregelmatige patronen en die de rol van fractals bij het verbeteren van de histologische classificatie van NSCLC hebben aangetoond.

Ten slotte werden in Hoofdstuk 8 de behoeften en technieken van systeemniveau onderzocht om een End-to-End diagnostisch Radiomics-model voor klinische toepassing te bouwen, en werden goede governance- en implementatiestrategieën voor data en AI-modellen in de ziekenhuizen besproken. Tot slot geeft hoofdstuk 9 een algemene bespreking van de resultaten die in dit proefschrift worden gepresenteerd en gerelateerde toekomstperspectieven op het gebied van automatisering in de gezondheidszorg. 


\section{VALORIZATION ADDENDUM}

With the increasing burden on healthcare both in terms of patient load as well as tremendous growth of the imaging data, there is a need for the healthcare industry to adopt advanced and newer solutions to improve the patient experience, affordability and access to care. These new technologies are already facilitating these transformations [1].

Royal Philips being a leading health technology company is focused on improving people's health and enabling better outcomes across the health continuum from healthy living, prevention, to diagnosis, treatment and home care. The vision of Philips is to improve the lives of 3 billion people a year by 2030 [2].

As far as the research presented in this thesis is concerned, being an employee of Philips the work in this thesis has influenced and contributed to the products and solutions as described in the paragraphs below.

\section{Knowledge Dissemination}

The research carried out in this thesis was shared with broader scientific community by publishing papers in Journals, book chapters and conferences. In addition, the concepts and the algorithms developed as part of thesis were shared amongst the researches across the Big Imaging data approach for Oncology in Netherlands India Collaboration (BIONIC) consortium. The algorithm for auto segmentation of lung and GTV is being used in the clinical setting of Tata Memorial Hospital in the radiomics extraction pipeline to realize the objectives of BIONIC. Further, the work on histology classification and new addition of fractals as features would help in identifying tumor habitats within the gross tumor volume. Finding of these habitats would be useful in targeted therapy for better prognosis. 


\section{Economical Exploitation}

The outcome of this thesis work has contributed towards addressing the need of hospitals and patients through the proposed solutions, in particular to cancer care. Philips being a health technology company can valorize the following results of this thesis.

1. The concepts and techniques proposed in Chapter 2, on how to build prognostic and predictive models and the challenges associated, can be part of Philips quality assurance framework for AI.

2. The Lung auto segmentation model using Deep Learning is being considered to be part of data science platform of Philips. Further, along with the Gross Tumor Volume detection is integrated into Philips Translation Research platform - IntelliSpace Discovery [3].

3. The Radiomic models developed in chapters 5, 6 and 7, survival models, automatic classification of tumor histopathology and Fractal Analysis for non-small lung cancer, are currently being verified and validated as part of the Philips Translation Research platform - IntelliSpace Discovery. Based on the outcome of the validation phase, the models could become part of the Philips IntelliSpace Portal [4], in the near future

4. The use cases discussed and developed in Chapter 8 a cloud-based framework to distribute/share clinical images with a CDSS for radiologists on the move and to the institutions that do not have an on-premise solutions can be part of a Philips cloudbased solution for distributed learning. 


\section{Societal Expectation Management}

Although, the work provided insights on the economic benefit and the dissemination of information to the scientific community, it also has influence on society. The technology blocks developed will help alleviate the burden of the physician thereby improve disease diagnosis and treatment at a rapid pace.

At the remote care setting, the cloud-based platform for image analytics, described in Chapter 8 , can provide capabilities, to help connect the experienced radiologists practicing in the large cities to physicians in remote villages and towns.

Further, the clinical decision support systems deployed on a cloud-based platform can empower physicians and healthcare workers in primary care to improve their diagnosis, treatment strategies and throughput.

\section{References}

[1] 2019 Global health care outlook-Shaping the future. https://www2.deloitte.com/content/dam/Deloitte/global/Documents/Life-Sciences-Health-Care/gx-Ishchc-outlook-2019.pdf

[2]. $\quad$ https://www.philips.com/a-w/about/company.htmI

[3]. IntelliSpace Discovery :

https://philipsproductcontent.blob.core.windows.net/assets/20190515/509c084a1c25484380c9aa4e00 cb6773.pdf

[4]. IntelliSpace Portal : https://www.philips.co.in/healthcare/product/HC881062/intellispace-portal-80-allyour-advanced-analysis-needs-one-comprehensive-solution 


\section{Curriculum Vitae}

Ravindra B Patil was born on $28^{\text {th }}$ June 1987 in Karnataka, India. After completion of his schooling from Military Residential School, Belgaum, Karnataka in 2004, he studied Electronics and Communication at VTU University and he graduated with University rank and Founder's gold Medal in 2008. Post his graduation, he joined Siemens Healthcare, India as campus recruit and worked as research engineer on image and signal processing based projects. This research exposure motivated him to pursue advanced degree. In 2011, he qualified the graduate aptitude test in engineering (GATE) and joined MS program at Indian Institute of Technology, Madras, India, where his work was focused on application of Pattern recognition and AI models on Diffusion Tensor Imaging for diagnosis of Alzheimer's. Post his masters in 2013, he joined Philips Research Lab, Bangalore as research scientist. In 2015, he started his PhD degree program with Maastro, Maastricht University, Netherlands under guidance of Prof. dr. ir. Andre Dekker and Dr. Leonard Wee. The work done as part of his thesis on Prognostic and Prediction Modelling with Radiomics for Non-Small Cell Lung Cancer has influenced and benefited similar projects at Philips Research. 


\section{Acknowledgments}

It is a pleasure to thank the people who made this thesis possible by offering their valuable suggestions, timely help and support.

First, I would like to thank my supervisor Prof. Dr. Andre Dekker for the insightful discussions, constructive criticism and his continuous support and encouragement during the research period and development of ideas in this thesis. He showed me different ways to approach a research problem and the need to be persistent to accomplish a goal. I shall always cherish the interaction with him and his approach towards addressing research problems with hands on knowledge.

I thank my co-supervisor Dr. Leonard Wee for his guidance, ever approachable nature and having critical and open discussions on the scientific methodologies. In addition, I wish to place special thanks for his rigorous and comprehensive review along with candid feedback on this thesis.

I would like to thank all my co-authors from Philips Research and Maastricht University, with whom I enjoyed working and having deep scientific discussions that helped me in improving my scientific acumen.

My special mention to Dr. Geetha M, with whom I share my PhD work, having co-located, helped in discussions, hospital visits, knowing the process and helping me in guiding across different challenges faced during PhD journey.

I wish to thank Dr. Dinesh M with whom I collaborated on BIONIC project, where some of the solution outcome of this thesis are part. I appreciate his help and scientific discussions in this journey.

In particular, want to thank, Nagaraju Bussa for the support, Allmin S, Chaitanya Kulkarni, Zhenwei Shi, Dr. Shyam Vasudev Rao for their collaboration.

Prognostic and Prediction Modelling with Radiomics for Non-Small Cell Lung Cancer $\mid 165$ 
I thank all my colleagues in Philips Research and Maastricht University who have directly and indirectly helped me with my thesis.

My deep and sincere gratitude to my family for their continuous and unparalleled love, help and support. I am extremely grateful to my Dad and Mom for their ever-inspiring attitude and support. Above all, I wish to thank my wife Dr. Vinutha, with whom I could discuss about the work and get clinical perspective of the challenges and for keeping me sane over the past few months. Thank you for being my proofreader, editor and sounding board! I appreciate, my little girl Saanvi for abiding my ignorance and the patience she showed during my thesis writing. Special mention to my sister and in-laws for their moral support.

I thank God, for having made everything possible by giving me strength and courage to do this work. 


\section{Chapter 11}

List of Publications

Ravindra Patil

Prognostic and Prediction Modelling with Radiomics for Non-Small Cell Lung Cancer | 167 


\section{Publications List}

Ravindra Patil, Geetha Mahadevaiah and Andre Dekker, "An Approach toward Automatic Classification of Tumor Histopathology of Non-Small Cell Lung Cancer Based on Radiomic Features" Jr.Tomography. 2016;2(4):374-377. doi:10.18383/j.tom.2016.00244.

Ravindra Patil, Geetha M, Andre Dekker, "Does Radiomics Improves Survival Prediction of NSCLC ? " Journal of Thoracic Oncology, November 2017 Volume 12, Issue 11, Supplement 2, Page S2089, DOI: https://doi.org/10.1016/j.jtho.2017.09.1154.

Ravindra Patil, Geetha Mahadevaiah, Srinidhi Bhat, Dinesh M.S, Leonard Wee and Andre Dekker, "Fractal Analysis in Histology Classification of Non-Small Cell Lung Cancer" Medical Imaging Artificial Intelligence, Image Recognition, and Machine Learning Techniques,2019, pp. 64-75 DOI: https://doi.org/10.1201/9780429029417.

Ravindra Patil, Wee L., Dekker A. (2020) Auto Segmentation of Lung in Non-small Cell Lung Cancer Using Deep Convolution Neural Network. Advances in Computing and Data Sciences. ICACDS. Communications in Computer and Information Science, 2020, vol 1244. Springer, DOI: https://doi.org/10.1007/978-981-15-6634-9_31

Prasad R V, Ravindra Patil, Ashish Jha, Sneha Mithun, Venkatesh Rangarajan , Leonard Wee and Andre Dekker, "Using Artificial Intelligence and Radiomics to support cancer care decisions" Using Artificial Intelligence and Radiomics to support cancer care decisions, 19th International Conference on the use of Computers in Radiation Therapy (2019).

Ravindra Patil, Leonard Wee, Andre Dekker, "Methodology in Prognostic and Prediction Modelling with Radiomics for Non-Small Cell Lung Cancer". Accepted for International Journal of Precision Oncology (in press)

Ravindra Patil, Leonard Wee and Andre Dekker, "Auto Segmentation of Gross Tumor Volume for Nsclc Using Deep Convolution Neural Network", Proceedings of Machine Learning Research part of arXiv ${ }^{\otimes}$ and part of International Conference on Medical Imaging Technologies and Radiology, 2020 